\title{
Hypergeometric Type Functions and Their Symmetries
}

\author{
Jan Dereziński
}

\begin{abstract}
The paper is devoted to a systematic and unified discussion of various classes of hypergeometric type equations: the hypergeometric equation, the confluent equation, the $F_{1}$ equation (equivalent to the Bessel equation), the Gegenbauer equation and the Hermite equation. In particular, recurrence relations of their solutions, their integral representations and discrete symmetries are discussed.
\end{abstract}

\section{Contents}

1. Introduction 1572

1.1. Classification 1572

1.2. Properties of Hypergeometric Type Operators 1574

1.3. Hypergeometric Type Functions 1575

$\begin{array}{ll}\text { 1.4. Degenerate Case } & 1577\end{array}$

$\begin{array}{ll}\text { 1.5. Canonical Forms } & 1577\end{array}$

1.6. Hypergeometric Type Polynomials 1578

$\begin{array}{ll}\text { 1.7. Parametrization } & 1579\end{array}$

1.8. Group-Theoretical Background 1580

1.9. Comparison with the Literature 1581

2. Preliminaries 1582

2.1. Differential Equations 1582

2.2. The Principal Branch of the Logarithm and the Power
Function

$\begin{array}{ll}\text { 2.3. Contours } & 1583\end{array}$

2.4. Reflection Invariant Differential Equations 1585

2.5. Regular Singular Points 1585

2.6. The Gamma Function 1586

2.7. The Pochhammer Symbol 1588

3. The ${ }_{2} F_{1}$ or the Hypergeometric Equation 1588

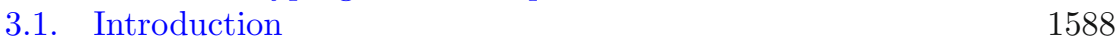


3.2. Integral Representations $\quad 1589$

3.3. Symmetries 1589

3.4. Factorization and Commutation Relations 1590

3.5. Canonical Forms 1592

3.6. The Hypergeometric Function 1593

3.7. Standard Solutions: Kummer's Table 1594

3.7.1. Solution $\sim 1$ at $0 \quad 1595$

3.7.2. Solution $\sim z^{-\alpha}$ at $0 \quad 1595$

3.7.3. Solution $\sim 1$ at $1 \quad 1595$

3.7.4. Solution $\sim(1-z)^{-\beta}$ at $1 \quad 1596$

3.7.5. Solution $\sim z^{-a}$ at $\infty \quad 1596$

3.7.6. Solution $\sim z^{-b}$ at $\infty \quad 1597$

$\begin{array}{ll}\text { 3.8. Connection Formulas } & 1597\end{array}$

3.9. Recurrence Relations 1598

3.10. Additional Recurrence Relations 1599

3.11. Degenerate Case 1599

3.12. Jacobi Polynomials 1600

$\begin{array}{ll}\text { 3.13. Special Cases } & 1604\end{array}$

3.13.1. Gegenbauer Equation Through an Affine

Transformation 1604

3.13.2. Gegenbauer Equation Through a Quadratic

Transformation $\quad 1605$

3.13.3. Chebyshev Equation $\quad 1605$

3.13.4. Legendre Equation 1605

3.13.5. Elementary Solutions $\quad 1605$

3.13.6. Fully Degenerate Case $\quad 1605$

4. The ${ }_{1} F_{1}$ and ${ }_{2} F_{0}$ Equation 1606

4.1. The ${ }_{1} F_{1}$ Equation 1606

4.2. The ${ }_{2} F_{0}$ Equation 1606

4.3. Equivalence of the ${ }_{1} F_{1}$ and ${ }_{2} F_{0}$ Equation 1606

4.4. Lie-Algebraic Parameters 1606

$\begin{array}{ll}\text { 4.5. Integral Representations } & 1607\end{array}$

4.6. Symmetries 1608

4.7. Factorizations and Commutation Relations 1608

$\begin{array}{lr}\text { 4.8. Canonical Forms } & 1609\end{array}$

4.9. The ${ }_{1} F_{1}$ Function 1610

4.10. The ${ }_{2} F_{0}$ Function 1611

4.11. Standard Solutions 1612

4.11.1. Solution $\sim 1$ at $0 \quad 1612$

4.11.2. Solution $\sim z^{-\alpha}$ at $0 \quad 1613$

4.11.3. Solution $\sim z^{-a}$ at $+\infty \quad 1613$

4.11.4. Solution $\sim(-z)^{-b} \mathrm{e}^{z}$ at $-\infty \quad 1613$

4.12. Connection Formulas 1614

4.13. Recurrence Relations 1614

4.14. Additional Recurrence Relations $\quad 1615$

$\begin{array}{ll}\text { 4.15. Degenerate Case } & 1615\end{array}$ 
$\begin{array}{ll}\text { 4.16. Laguerre Polynomials } & 1615\end{array}$

$\begin{array}{ll}\text { 4.17. Bessel Polynomials } & 1617\end{array}$

$\begin{array}{ll}\text { 4.18. Special Cases } & 1618\end{array}$

4.18.1. Bessel Equation 1619

4.18.2. Hermite Equation 1619

5. The ${ }_{0} F_{1}$ Equation 1619

$\begin{array}{ll}\text { 5.1. Introduction } & 1619\end{array}$

5.2. Equivalence with a Subclass of the Confluent Equation 1619

5.3. Integral Representations 1619

5.4. Symmetries 1621

5.5. Factorizations and Commutation Relations 1621

5.6. Canonical Forms 1621

5.7. The ${ }_{0} F_{1}$ Function 1622

5.8. Standard Solutions 1623

5.8.1. Solution $\sim 1$ at $0 \quad 1623$

5.8.2. Solution $\sim z^{-\alpha}$ at $0 \quad 1623$

5.8.3. Solution $\sim \exp \left(-2 z^{\frac{1}{2}}\right) z^{-\frac{\alpha}{2}-\frac{1}{4}}$ for $z \rightarrow+\infty \quad 1624$

5.9. Connection Formulas $\quad 1625$

5.10. Recurrence Relations $\quad 1625$

5.11. Degenerate Case 1625

5.12. Special Cases $\quad 1626$

6. The Gegenbauer Equation 1626

$\begin{array}{lr}\text { 6.1. Introduction } & 1626\end{array}$

6.2. Equivalence with the Hypergeometric Equation 1626

$\begin{array}{ll}\text { 6.3. Symmetries } & 1627\end{array}$

6.4. Factorizations and Commutation Relations 1628

6.5. The Riemann Surface of the Gegenbauer Equation 1629

$\begin{array}{lr}\text { 6.6. Integral Representations } & 1630\end{array}$

6.7. Canonical Forms 1631

6.8. Even Solution 1631

6.9. Odd Solution 1632

6.10. Standard Solutions 1632

6.10.1. Solution $\sim 1$ at $1 \quad 1633$

6.10.2. Solution $\sim 2^{-\alpha}(1-z)^{-\alpha}$ at $1 \quad 1634$

6.10.3. Solution $\sim z^{-a}$ at $\infty \quad 1634$

6.10.4. Solution $\sim z^{-b}$ at $\infty \quad 1635$

6.11. Connection Formulas $\quad 1635$

6.12. Recurrence Relations 1636

$\begin{array}{lr}\text { 6.13. Gegenbauer Polynomials } & 1636\end{array}$

6.14. Special Cases 1639

6.14.1. The Legendre Equation 1639

6.14.2. Chebyshev Equation of the 1st Kind 1640

6.14.3. Chebyshev Equation of the 2nd Kind 1640

7. The Hermite Equation 1641

$\begin{array}{ll}\text { 7.1. Introduction } & 1641\end{array}$

7.2. Equivalence with a Subclass of the Confluent Equation 1642 
7.3. Symmetries

7.4. Factorizations and Commutation Properties

7.5. Convergence of the Gegenbauer Equation to the Hermite Equation

7.6. Integral Representations

7.7. Canonical Forms

7.8. Even Solution

7.9. Odd Solution

7.10. Standard Solutions

7.10.1. Solution $\sim z^{-a}$ for $z \rightarrow+\infty$

7.10.2. Solution $\sim(-\mathrm{i} z)^{a-1} \mathrm{e}^{z^{2}}$ for $z \rightarrow+\mathrm{i} \infty$

7.11. Connection Formulas

7.12. Recurrence Relations

$\begin{array}{ll}\text { 7.13. Hermite Polynomials } & 1647\end{array}$

$\begin{array}{lr}\text { Acknowledgements } & 1648\end{array}$

$\begin{array}{lr}\text { Open Access } & 1648\end{array}$

Appendix A. Contours for Integral Representations 1648

References

\section{Introduction}

Following [10], we adopt the following terminology. Equations of the form

$$
\left(\sigma(z) \partial_{z}^{2}+\tau(z) \partial_{z}+\eta\right) f(z)=0
$$

where $\sigma$ is a polynomial of degree $\leq 2, \tau$ is a polynomial of degree $\leq 1, \eta$ is a number, will be called hypergeometric type equations, and their solutions - hypergeometric type functions. Differential operators of the form $\sigma(z) \partial_{z}^{2}+$ $\tau(z) \partial_{z}+\eta$ will be called hypergeometric type operators.

The theory of hypergeometric type functions is one of the oldest and most useful chapters of mathematics. In usual presentations, it appears complicated and messy. The main purpose of this paper is an attempt to present its basics in a way that shows clearly its internal structure and beauty.

\subsection{Classification}

Let us start with a short review of basic classes of hypergeometric type equations. We will always assume that $\sigma(z) \neq 0$. Every class, except for (9), will be simplified by dividing by a constant and an affine change of the complex variable $z$.

(1) The ${ }_{2} F_{1}$ or hypergeometric equation

$$
\left(z(1-z) \partial_{z}^{2}+(c-(a+b+1) z) \partial_{z}-a b\right) f(z)=0 .
$$

(2) The ${ }_{2} F_{0}$ equation

$$
\left(z^{2} \partial_{z}^{2}+(1+(1+a+b) z) \partial_{z}+a b\right) f(z)=0 .
$$


(3) The ${ }_{1} F_{1}$ or confluent equation

$$
\left(z \partial_{z}^{2}+(c-z) \partial_{z}-a\right) f(z)=0
$$

(4) The ${ }_{0} F_{1}$ equation

$$
\left(z \partial_{z}^{2}+c \partial_{z}-1\right) f(z)=0
$$

(5) The Gegenbauer equation

$$
\left(\left(1-z^{2}\right) \partial_{z}^{2}-(a+b+1) z \partial_{z}-a b\right) f(z)=0 .
$$

(6) The Hermite equation

$$
\left(\partial_{z}^{2}-2 z \partial_{z}-2 a\right) f(z)=0
$$

(7) 2nd order Euler equation

$$
\left(z^{2} \partial_{z}^{2}+b z \partial_{z}+a\right) f(z)=0 .
$$

(8) 1st order Euler equation for the derivative

$$
\left(z \partial_{z}^{2}+c \partial_{z}\right) f(z)=0 .
$$

(9) 2nd order equation with constant coefficients

$$
\left(\partial_{z}^{2}+c \partial_{z}+a\right) f(z)=0
$$

One can divide these classes into three families:

1. (1), (2), (3), (4);

2. (5), (6);

3. (7), (8), (9).

Each equation in the first family has a solution equal to the hypergeometric function ${ }_{p} F_{q}$ with appropriate $p, q$. This function gives a name to the corresponding class of equations.

The second family consists of reflection invariant equations.

The third family consists of equations solvable in elementary functions. Therefore, it will not be considered in what follows.

The ${ }_{2} F_{0}$ and ${ }_{1} F_{1}$ equation are equivalent by a simple substitution; therefore, they can be discussed together.

Up to an affine transformation, (5) is a subclass of (1). However, it has additional properties; therefore, it is useful to discuss it separately.

The main part of our paper consists of 5 sections corresponding to the classes (1), (2)-(3), (4), (5) and (6). The discussion will be divided into two levels:

1. Properties of the operator that defines the equation.

2. Properties of functions solving the equation. 


\subsection{Properties of Hypergeometric Type Operators}

We will discuss the following types of properties of hypergeometric type operators:

(i) equivalence between various classes,

(ii) integral representations of solutions,

(iii) discrete symmetries,

(iv) factorizations,

(v) commutation relations.

Let us give some examples of these properties. All these examples will be related to the ${ }_{1} F_{1}$ equation.

We have

$$
\begin{aligned}
& (-w)^{a+1}\left(w^{2} \partial_{w}^{2}+(-1+(1+a+b) w) \partial_{w}+a b\right) w^{-a} \\
& \quad=z \partial_{z}^{2}+(c-z) \partial_{z}-a, \quad w=-z^{-1}
\end{aligned}
$$

Therefore, the ${ }_{1} F_{1}$ operator, appearing in (1.3), is equivalent to the ${ }_{2} F_{0}$ operator, which is inside the brackets of (1.2). This is an example of (i).

As an example of (ii), we quote the following fact: The integral

$$
\int_{\gamma} t^{a-c} \mathrm{e}^{t}(t-z)^{-a} \mathrm{~d} t
$$

is a solution of the ${ }_{1} F_{1}$ equation provided that the values of the function

$$
t \mapsto t^{a-c+1} \mathrm{e}^{t}(t-z)^{-a-1}
$$

at the endpoints of the curve $\gamma$ are equal to one another.

Note that the integrand of (1.4) is an elementary function. The condition on the curve $\gamma$ can often be satisfied in a number of non-equivalent ways, giving rise to distinct natural solutions.

An example of (iii) is the following identity:

$$
\begin{aligned}
& w \partial_{w}^{2}+(c-w) \partial_{w}-a \\
& \quad=-\mathrm{e}^{-z}\left(z \partial_{z}^{2}+(c-z) \partial_{z}-c+a\right) \mathrm{e}^{z}, \quad w=-z .
\end{aligned}
$$

Thus, the ${ }_{1} F_{1}$ operator is transformed into a ${ }_{1} F_{1}$ operator with different parameters.

Here is a pair of examples of (iv):

$$
\begin{aligned}
& z\left(z \partial_{z}^{2}+(c-z) \partial_{z}-a\right) \\
& \quad=\left(z \partial_{z}+a-1\right)\left(z \partial_{z}+c-a-z\right)+(a-1)(a-c) \\
& \quad=\left(z \partial_{z}+c-a-1-z\right)\left(z \partial_{z}+a\right)+a(a+1-c) .
\end{aligned}
$$

An example of $(\mathrm{v})$ is

$$
\left(z \partial_{z}+a\right) z\left(z \partial_{z}^{2}+(c-z) \partial_{z}-a\right)=z\left(z \partial_{z}^{2}+(c-z) \partial_{z}-a-1\right)\left(z \partial_{z}+a\right) .
$$

On both sides of the identity, we see the ${ }_{1} F_{1}$ operators whose parameters are contiguous. 
The commutation properties can be derived from the factorizations. Let us show, for example, how (1.7) and (1.8) imply (1.9). First, we rewrite (1.7) as

$$
z\left(z \partial_{z}^{2}+(c-z) \partial_{z}-a-1\right)=\left(z \partial_{z}+a\right)\left(z \partial_{z}+c-a-1-z\right)+a(a+1-c) .
$$

Then, we multiply (1.8) from the left and (1.10) from the right by $\left(z \partial_{z}+a\right)$, obtaining identical right hand sides. This yields (1.9).

\subsection{Hypergeometric Type Functions}

After the analysis of hypergeometric type operators, we discuss hypergeometric type functions, that is, functions annihilated by hypergeometric type operators. In particular, we will distinguish the so-called standard solutions which have a simple behavior around a singular point of the equation. In particular, if $z_{0}$ is a regular singular point, the Frobenius method gives us two solutions behaving as $\left(z-z_{0}\right)^{\lambda_{i}}$, where $\lambda_{1}, \lambda_{2}$ are the indices of $z_{0}$. One can often find solutions with a simple behavior also around irregular singular points.

For reflection invariant classes (5) and (6), one can also define another pair of natural solutions: the even solution $S^{+}$, which we normalize by $S^{+}(0)$ $=1$, and the odd solution $S^{-}$, which we normalize by $\left(S^{-}\right)^{\prime}(0)=2$.

Discrete symmetries can be used to derive properties of hypergeometric type functions. For instance, (1.6) implies that if $f(z)$ solves the confluent equation for parameters $c-a, c$, then so does $\mathrm{e}^{z} f(-z)$ for the parameters $a, c$. In particular, both functions $F(a ; c ; z)$ and $\mathrm{e}^{z} F(c-a ; c ;-z)$ solve the confluent equation for the parameters $a, c$. Both are analytic around $z=0$ and equal 1 at $z=0$. By the uniqueness of the solution to the Frobenius method, they should coincide. Hence, we obtain the identity

$$
F(a ; c ; z)=\mathrm{e}^{z} F(c-a ; c ;-z) .
$$

Commutation relations are also useful. For example, it follows immediately from $(1.9)$ that $\left(z \partial_{z}+a\right) F(a ; c ; z)$ is a solution of the confluent equation for the parameters $a+1, c$. At zero, it is analytic and its value is $a$. Hence we obtain the recurrence relation

$$
\left(z \partial_{z}+a\right) F(a ; c ; z)=a F(a+1 ; c ; z) .
$$

For each class of equations, we describe a whole family of recurrence relations. Every such recurrence relation involves an operator of the following form: a 1st order differential operator with no dependence on the parameters + a multiplication operator depending linearly on the parameters. We will call them basic recurrence relations.

Sometimes, there also exist more complicated recurrence relations. We do not give their complete list, we only mention some of their examples. We call them additional recurrence relations.

Each of the standard solutions has simple integral representations of the form analogous to (1.4). Each of these integral representations is associated with a pair of (possibly infinite and possibly coinciding) points where the 
integrand has a singularity. We will use two basic kinds of contours for standard solutions:

(a) The contour starts at one singularity and ends at the other singularity; we assume that at both singularities the analog of (1.5) is zero (hence, trivially, has equal values).

(b) The contour starts at the first singularity, goes around the second singularity and returns to the first singularity; we assume that the analog of (1.5) is zero at the first singularity.

If available, we will always treat the type (a) contour as the basic one.

For instance, under appropriate conditions on the parameters, the ${ }_{1} F_{1}$ function has the following two integral representations:

$$
\begin{aligned}
\operatorname{type}(\mathrm{a}): \quad \int_{[1,+\infty[} \mathrm{e}^{\frac{z}{t}} t^{-c}(t-1)^{c-a-1} \mathrm{~d} t & =\frac{\Gamma(a) \Gamma(c-a)}{\Gamma(c)} F(a ; c ; z), \\
\operatorname{type}(\mathrm{b}): \frac{1}{2 \pi \mathrm{i}} \int_{\left[1,0^{+}, 1\right]} \mathrm{e}^{\frac{z}{t}}(-t)^{-c}(-t+1)^{c-a-1} \mathrm{~d} t & =\frac{\Gamma(c-a)}{\Gamma(1-a) \Gamma(c)} F(a ; c ; z) .
\end{aligned}
$$

$\left(0^{+}\right.$means that we bypass 0 in the counterclockwise direction; in this case, it is equivalent to bypassing $\infty$ in the clockwise direction).

There are various natural ways to normalize hypergeometric type functions. The most obvious normalization for a solution analytic at a given regular singular point is to demand that its value there is 1 . (For the ${ }_{2} F_{0}$ equation, the point 0 is not regular singular; however, there is a natural generalization of this normalization condition). For equations (1)-(4), this function will be denoted by the letter $F$, consistently with the conventional usage. (Note the use of the italic font). In the case of reflection symmetric equations (5) and (6), we will use the letter $S$.

However, it is often preferable to use different normalizations, which involve appropriate values of the Gamma function or its products. Such normalizations arise naturally when we consider integral representations. They will be denoted by $\mathbf{F}$ for equations (1)-(4) (a similar notation can be found in [12]), and $\mathbf{S}$ for (5) and (6). (Note the use of the boldface roman font). Sometimes, there will be several varieties of these normalizations denoted by an appropriate superscript, related to various integral representations. The functions with these normalizations have often better properties than the $F$ and $S$ functions. This is especially visible in recurrence relations, where the coefficient on the right (such as $a$ in (1.12)) depends on the normalization.

For example, for the ${ }_{1} F_{1}$ function, we introduce the following normalizations:

$$
\begin{aligned}
\mathbf{F}(a ; c ; z) & :=\frac{1}{\Gamma(c)} F(a ; c ; z), \\
\mathbf{F}^{\mathrm{I}}(a ; c ; z) & :=\frac{\Gamma(a) \Gamma(c-a)}{\Gamma(c)} F(a ; c ; z),
\end{aligned}
$$

the latter suggested by the type (a) integral representation given above. 


\subsection{Degenerate Case}

For some values of parameters, hypergeometric type functions have special properties. This happens, in particular, when the difference of the indices at a given regular singular point is an integer. Then, the two standard solutions related to this point are proportional to one another. We call them degenerate solutions. (The best known example of such a situation is the Bessel functions of integer parameters). In this case, we have a simple generating function and an additional integral representation, which involves integrating over a closed loop.

\subsection{Canonical Forms}

Obviously, hypergeometric type operators coincide with differential operators of the form

$$
\begin{aligned}
& \sigma(z) \partial_{z}^{2}+\left(\sigma^{\prime}(z)+\kappa(z)\right) \partial_{z}+\frac{1}{2} \kappa^{\prime}+\lambda \\
& =\partial_{z} \sigma(z) \partial_{z}+\frac{1}{2}\left(\partial_{z} \kappa(z)+\kappa(z) \partial_{z}\right)+\lambda
\end{aligned}
$$

where $\sigma$ is a polynomial of degree $\leq 2, \kappa$ is a polynomial of degree $\leq 1$, and $\lambda$ is a number.

One can argue that it is natural to use $\sigma, \kappa, \lambda$ to parametrize the hypergeometric type operators (more natural than $\sigma, \tau, \eta)$. Equation (1.13) will be denoted $\mathcal{C}\left(\sigma, \kappa, \lambda ; z, \partial_{z}\right)$, or, for brevity, $\mathcal{C}(\sigma, \kappa, \lambda)$. Let $\rho(z)$ be a solution of the equation

$$
\left(\sigma(z) \partial_{z}-\kappa(z)\right) \rho(z)=0 .
$$

(Note that Eq. (1.14) is solvable in elementary functions). We have the identity

$$
\mathcal{C}(\sigma, \kappa, \lambda)=\rho^{-1}(z) \partial_{z} \sigma(z) \rho(z) \partial_{z}+\frac{1}{2} \kappa^{\prime}+\lambda,
$$

We will call $\rho$ the natural weight. To justify this name note that if $\lambda$ is real, $\sigma, \kappa$ are real and $\rho$ is positive and nonsingular on $] a, b[\subset \mathbb{R}$, then $\mathcal{C}(\sigma, \kappa, \lambda)$ is Hermitian on the weighted space $L^{2}(] a, b[, \rho)$, when as the domain we take $C_{\mathrm{c}}^{\infty}(] a, b[)$.

It is sometimes useful to replace the operator $\mathcal{C}(\sigma, \kappa, \lambda)$ with

$$
\rho(z)^{\frac{1}{2}} \mathcal{C}(\sigma, \kappa, \lambda) \rho(z)^{-\frac{1}{2}}=\partial_{z} \sigma(z) \partial_{z}-\frac{\kappa(z)^{2}}{4 \sigma(z)}+\lambda .
$$

We will call (1.16) the balanced form of $\mathcal{C}(\sigma, \kappa, \lambda)$.

Sometimes, one replaces (1.1) by the 1-dimensional Schrödinger equation

$$
\left(\partial_{z}^{2}-V(z)\right) f=0
$$

where

$$
V(z):=\frac{1}{2}\left(\sigma(z)^{-1} \sigma^{\prime}(z)\right)^{\prime}+\frac{1}{4}\left(\sigma(z)^{-1} \sigma^{\prime}(z)\right)^{2}+\frac{\kappa(z)^{2}}{4 \sigma(z)^{2}}-\frac{\lambda}{\sigma(z)} .
$$

(1.17) is equivalent to (1.1), because

$$
\sigma(z)^{-\frac{1}{2}} \rho(z)^{\frac{1}{2}} \mathcal{C}(\sigma, \kappa, \lambda) \rho(z)^{-\frac{1}{2}} \sigma(z)^{-\frac{1}{2}}=\partial_{z}^{2}-V(z),
$$

It will be called the Schrödinger-type form of the equation $\mathcal{C}(\sigma, \kappa, \lambda) f=0$. 
Some of the symmetries of hypergeometric type equations are obvious in the balanced and Schrödinger-type forms. This is partly due to the fact that they do not change when we switch the sign in front of $\kappa$. This is a serious advantage of these forms.

In the literature, various forms of hypergeometric type equations are used. Instead of the Gegenbauer equation, one usually finds its balanced form, called the associated Legendre equation. The modified Bessel equation and the Bessel equation, equivalent to the rarely used ${ }_{0} F_{1}$ equation, are the balanced form of a special case of the ${ }_{1} F_{1}$ equation. Instead of the ${ }_{1} F_{1}$ equation, one often finds its Schrödinger-type form, the Whittaker equation. This usage, due mostly to historical traditions, makes the subject more complicated than necessary.

We will always use (1.1) as the basic form. Its main advantage is that in almost all cases the equation in the form (1.1) has at least one solution analytic around a given finite singular point. Even in the case of the ${ }_{2} F_{0}$ equation, whose all solutions have a branch point at 0 , there exists a distinguished solution particularly well behaved at zero.

\subsection{Hypergeometric Type Polynomials}

Hypergeometric type polynomials, that is, polynomial solutions of hypergeometric type equations deserve a separate analysis. They have traditional names involving various nineteenth century mathematicians. Note, in particular, that the (rarely used) polynomial cases of the ${ }_{2} F_{0}$ function are called Bessel polynomials; however, they do not have a direct relation to the better-known Bessel functions.

There exists a well-known elegant approach to their theory that allows us to derive most of their basic properties in a unified way, see e.g. [10,13]. Let us sketch this approach.

Fix $\sigma, \kappa, \rho$, as in Sect. 1.5. For any $n=0,1,2, \ldots$, we define

$$
P_{n}(\sigma, \rho ; z):=\frac{1}{n !} \rho^{-1}(z) \partial_{z}^{n} \rho(z) \sigma^{n}(z) .
$$

We will call (1.19) a Rodriguez-type formula, since it is a generalization of the Rodriguez formula for Legendre polynomials.

One can show that $P_{n}$ solves the equation

$$
\left(\sigma(z) \partial_{z}^{2}+\left(\sigma^{\prime}(z)+\kappa(z)\right) \partial_{z}-n(n+1) \frac{\sigma^{\prime \prime}}{2}-n \kappa^{\prime}\right) P_{n}(\sigma, \rho ; z)=0
$$

$P_{n}$ is a polynomial, typically of degree $n$, more precisely its degree is given as follows:

1. If $\sigma^{\prime \prime}=\kappa^{\prime}=0$, then $\operatorname{deg} P_{n}=0$.

2. If $\sigma^{\prime \prime} \neq 0$ and $-\frac{2 \kappa^{\prime}}{\sigma^{\prime \prime}}-1=m$ is a positive integer, then

$$
\operatorname{deg} P_{n}= \begin{cases}n, & n=0,1, \ldots, m ; \\ n-m-1, & n=m+1, m+2, \ldots\end{cases}
$$

3. Otherwise, $\operatorname{deg} P_{n}=n$. 
We have a generating function

$$
\frac{\rho(z+t \sigma(z))}{\rho(z)}=\sum_{n=0}^{\infty} t^{n} P_{n}\left(\sigma, \rho \sigma^{-n} ; z\right),
$$

an integral representation

$$
P_{n}(\sigma, \rho ; z)=\frac{1}{2 \pi \mathrm{i}} \rho^{-1}(z) \int_{\left[z^{+}\right]} \sigma^{n}(z+t) \rho(z+t) t^{-n-1} \mathrm{~d} t
$$

and recurrence relations

$$
\begin{aligned}
\left(\sigma(z) \partial_{z}+\left(\kappa(z)-n \sigma^{\prime}(z)\right) P_{n}\left(\sigma, \rho \sigma^{-n} ; z\right)\right. & =P_{n+1}\left(\sigma, \rho \sigma^{-n-1} ; z\right), \\
\partial_{z} P_{n+1}\left(\sigma, \rho \sigma^{-n-1} ; z\right) & =\left(-n \frac{\sigma^{\prime \prime}}{2}+\kappa^{\prime}\right) P_{n}\left(\sigma, \rho \sigma^{-n} ; z\right) .
\end{aligned}
$$

In almost all sections, we devote a separate subsection to the corresponding class of polynomials. Beside the properties that follow immediately from the unified theory presented above, we describe additional properties valid in a given class.

The ${ }_{0} F_{1}$ equation does not have polynomial solutions, hence the corresponding section is the only one without a subsection about polynomials.

Another special situation arises in the case of the Gegenbauer equation. The standard Gegenbauer polynomials found in the literature do not have the normalization given by the Rodriguez-type formula. The Rodriguez-type formula yields the Jacobi polynomials, which for $\alpha=\beta$ coincide with the Gegenbauer polynomials up to a nontrivial coefficient. Thus, for the Gegenbauer equation, it is natural to consider two classes of polynomials differing by normalization. This is related to an interesting symmetry called the Whipple transformation, which is responsible for two kinds of integral representations.

\subsection{Parametrization}

Each class (1)-(6) depends on a number of complex parameters, denoted by Latin letters belonging to the set $\{a, b, c\}$. They will be called the classical parameters. They are convenient when we discuss power series expansions of standard solutions.

Unfortunately, the classical parameters are not convenient to describe discrete symmetries. Therefore, for each class (1)-(6), we introduce an alternative set of parameters, which we will call the Lie-algebraic parameters. They will be denoted by Greek letters such as $\alpha, \beta, \mu, \theta, \lambda$, and will be given by certain linear (possibly, inhomogeneous) combinations of the classical parameters. Discrete symmetries of hypergeometric type equations will simply involve signed permutations of the Lie algebraic parameters - in the classical parameters, they look much more complicated. Recurrence relations also become simpler in the Lie-algebraic parameters.

For polynomials of hypergeometric type a third kind of parametrization is traditionally used. They are characterized by their degree $n$, which coincides with $-a$, where $a$ is one of the classical parameters. The Lie-algebraic 
parameters appearing inside the 1st order part of the equation are used as the remaining parameters.

Let us stress that all these parametrizations are natural and useful. Therefore, we sometimes face the dilemma which parametrization to use for a given set of identities. We usually try to choose the one that gives the simplest formulas.

We sum up the information about various parametrizations in the following table:

\begin{tabular}{|c|c|c|c|c|}
\hline Equation & $\begin{array}{l}\text { classical } \\
\text { parameters }\end{array}$ & $\begin{array}{l}\text { Lie-algebraic } \\
\text { parameters }\end{array}$ & Polynomial & $\begin{array}{l}\text { parameters for } \\
\text { polynomials }\end{array}$ \\
\hline${ }_{2} F_{1}$ & $a, b, c$ & $\begin{array}{l}\alpha=c-1 \\
\beta=a+b-c \\
\gamma=b-a\end{array}$ & Jacobi & $\begin{array}{l}\alpha=c-1 \\
\beta=a+b-c \\
n=-a\end{array}$ \\
\hline${ }_{2} F_{0}$ & $a, b$ & $\begin{array}{l}\theta=-1+a+b \\
\alpha=a-b\end{array}$ & Bessel & $\begin{array}{l}\theta=-1+a+b \\
n=-a\end{array}$ \\
\hline${ }_{1} F_{1}$ & $a, c$ & $\begin{array}{l}\theta=-c+2 a \\
\alpha=c-1\end{array}$ & Laguerre & $\begin{array}{l}\alpha=c-1 \\
n=-a\end{array}$ \\
\hline${ }_{0} F_{1}$ & $c$ & $\alpha=c-1$ & ----- & ----- \\
\hline Gegenbauer & $a, b$ & $\begin{array}{l}\alpha=\frac{a+b-1}{2} \\
\lambda=\frac{b-a}{2}\end{array}$ & $\begin{array}{l}\alpha=\beta \text { Jacobi } \\
\text { or Gegenbauer }\end{array}$ & $\begin{array}{l}\alpha=\frac{a+b-1}{2} \\
n=-a\end{array}$ \\
\hline Hermite & $a$ & $\lambda=a-\frac{1}{2}$ & Hermite & $n=-a$ \\
\hline
\end{tabular}

\subsection{Group-Theoretical Background}

Identities for hypergeometric type operators and functions have a high degree of symmetry. Therefore, it is natural to expect that a certain group-theoretical structure is responsible for these identities.

There exists a large literature about the relations between special functions and the group theory $[6,8,15,16]$. Nevertheless, as far as we know, the arguments found in the literature give a rather incomplete explanation of the properties that we describe. In a separate publication [2], we would like to present a group-theoretical approach to hypergeometric type functions with, we believe, a more satisfactory justification of their high symmetry. Below, we would like to briefly sketch the main ideas of [2].

Each hypergeometric type equation can be obtained by separating the variables of a certain 2nd order PDE of the complex variable with constant coefficients. One can introduce the Lie algebra of generalized symmetries of this PDE. In this Lie algebra, we fix a certain maximal commutative algebra, which we will call the "Cartan algebra". Operators whose adjoint action is diagonal in the "Cartan algebra" will be called "root operators". Automorphisms of the Lie algebra leaving invariant the "Cartan algebra" will be called "Weyl symmetries".

(Note that in some cases, the Lie algebra of symmetries is simple, and then the names Cartan algebra, root operators and Weyl symmetries correspond to the standard names. In other cases the Lie algebra is non-semisimple, and then the names are less standard - this is the reason for the quotation marks that we use). 
Now the parameters of hypergeometric type equation can be interpreted as the eigenvalues of elements of the "Cartan algebra". In particular, the Lie algebraic parameters correspond to a certain natural choice of the "Cartan algebra". Each recurrence relation is related to a "root operator". Finally, each symmetry of a hypergeometric type operator corresponds to a Weyl symmetry of the Lie algebra.

We can distinguish three kinds of PDE's with constant coefficients:

1. The Helmholtz equation on $\mathbb{C}^{n}$ given by $\Delta_{n}+1$, whose Lie algebra of symmetries is $\mathbb{C}^{n} \rtimes s o(n, \mathbb{C})$;

2. The Laplace equation on $\mathbb{C}^{n}$ given by $\Delta_{n}$, whose Lie algebra of generalized symmetries is $s o(n+2, \mathbb{C})$

3. The heat equation on $\mathbb{C}^{n} \oplus \mathbb{C}$ given by $\Delta_{n}+\partial_{s}$, whose Lie algebra of generalized symmetries is $\operatorname{sch}(n, \mathbb{C})$ (the so-called (complex) Schrödinger Lie algebra.

Separating the variables in these equations usually leads to differential equations with many variables. Only in a few cases, it leads to ordinary differential equations, which turn out to be of hypergeometric type. Here is a table of these cases:

\begin{tabular}{|l|l|l|l|l|}
\hline PDE & Lie algebra & $\begin{array}{l}\text { dimension of } \\
\text { Cartan algebra }\end{array}$ & $\begin{array}{l}\text { discrete } \\
\text { symmetries }\end{array}$ & equation \\
\hline$\Delta_{2}+1$ & $\mathbb{C}^{2} \rtimes s o(2, \mathbb{C})$ & 1 & $\mathbb{Z}_{2}$ & ${ }_{0} F_{1} ;$ \\
$\Delta_{4}$ & $s o(6, \mathbb{C})$ & 3 & cube & ${ }_{2} F_{1} ;$ \\
$\Delta_{3}$ & $s o(5, \mathbb{C})$ & 2 & square & Gegenbauer; \\
$\Delta_{2}+\partial_{s}$ & $s c h(2, \mathbb{C})$ & 2 & $\mathbb{Z}_{2} \times \mathbb{Z}_{2}$ & ${ }_{1} F_{1}$ or ${ }_{2} F_{0} ;$ \\
$\Delta_{1}+\partial_{s}$ & $s c h(1, \mathbb{C})$ & 1 & $\mathbb{Z}_{4}$ & Hermite. \\
\hline
\end{tabular}

\subsection{Comparison with the Literature}

There exist many works that discuss hypergeometric type functions, e.g. $[1,4,7$, 11-14,17]. Some of them are meant to be encyclopedic collections of formulas, others try to show mathematical structure that underlies their properties.

In our opinion, this work differs substantially from the existing literature. In our presentation, we try to follow the intrinsic logic of the subject, without too much regard for the traditions. If possible, we apply the same pattern to each class of hypergeometric type equations. This sometimes forces us to introduce unconventional notation.

We believe that the intricacy of usual presentations of hypergeometric type functions can be partly explained by historical reasons. In the literature, various classes of these functions are often described with help of different conventions. Sometimes, we will give short remarks devoted to the conventions found in the literature. These remarks will always be clearly separated from the main text.

Of course, our presentation does not contain all useful identities and properties of hypergeometric functions. Some of them are on purpose left out, e.g. the so-called addition formulas. We restrict ourselves to what we view as 
the most basic theory. On the other hand, we try to be complete for each type of properties that we consider.

Our work is strongly inspired by the book by Nikiforov and Uvarov [10], who tried to develop a unified approach to hypergeometric type functions. They stressed, in particular, the role of integral representations and of recurrence relations.

Another important influence is the works of Miller [8,9] who stressed the Lie-algebraic structure behind the recurrence relations.

The method of factorization can be traced back at least to [5].

\section{Preliminaries}

In this section, we fix basic terminology, notation and collect a number of well known useful facts, mostly from complex analysis. It is supposed to serve as a reference and can be skipped at the first reading.

\subsection{Differential Equations}

The main objectives of our paper are ordinary homogeneous 2nd order linear differential equations in the complex domain, that is equations of the form

$$
\left(a(z) \partial_{z}^{2}+b(z) \partial_{z}+c(z)\right) \phi(z)=0 .
$$

It will be convenient to treat (2.1) as the problem of finding the kernel of the operator

$$
\mathcal{A}\left(z, \partial_{z}\right):=a(z) \partial_{z}^{2}+b(z) \partial_{z}+c(z) .
$$

We will then say that the Eq. (2.1) is given by the operator (2.2). When we do not consider the change of the variable, we will often write $\mathcal{A}$ for $\mathcal{A}\left(z, \partial_{z}\right)$.

\subsection{The Principal Branch of the Logarithm and the Power Function}

The function

$$
\left.\left.\{z \in \mathbb{C}:-\pi<\operatorname{Im} z<\pi\} \ni z \mapsto \mathrm{e}^{z} \in \mathbb{C} \backslash\right]-\infty, 0\right]
$$

is bijective. Its inverse will be called the principal branch of the logarithm and will be denoted simply $\log z$.

If $\mu \in \mathbb{C}$ then the principal branch of the power function is defined as

$$
\mathbb{C} \backslash]-\infty, 0] \ni z \mapsto z^{\mu}:=\mathrm{e}^{\mu \log z} .
$$

Consequently, if $\alpha \in \mathbb{C} \backslash\{0\}$, then the functions $\log \left(\alpha\left(z-z_{0}\right)\right)$ and $\left(\alpha\left(z-z_{0}\right)\right)^{\mu}$ have the domain $\left.\left.\mathbb{C} \backslash\left(z_{0}+\alpha^{-1}\right]-\infty, 0\right]\right)$.

Of course, if needed we will use the analytic continuation to extend the definition of the logarithm and the power function beyond $\mathbb{C} \backslash]-\infty, 0]$ onto the appropriate covering of $\mathbb{C} \backslash\{0\}$. 


\subsection{Contours}

We will write

$$
\left.f(z)\right|_{z_{0}} ^{z_{1}}:=f\left(z_{1}\right)-f\left(z_{0}\right)
$$

In particular, if $] 0,1[\ni t \mapsto \gamma(t) \in \mathbb{C}$ is a curve, then

$$
\left.f(z)\right|_{\gamma(0)} ^{\gamma(1)}=\int_{\gamma} f^{\prime}(z) \mathrm{d} z .
$$

To avoid making pictures, we will use special notation for contours of integration.

Broken lines will be denoted as in the following example:

$$
\left[w_{0}, u, w_{1}\right]:=\left[w_{0}, u\right] \cup\left[u, w_{1}\right] .
$$

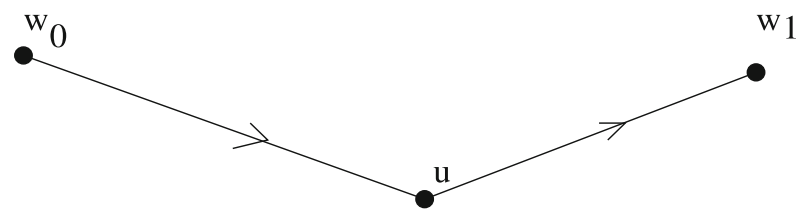

This contour may be inappropriate if the function has a nonintegrable singularity at $u$. Then, we might want to bypass $u$ with a small arc counterclockwise or clockwise. In such a case, we can use the curves

$$
\left[w_{0}, u^{+}, w_{1}\right]
$$

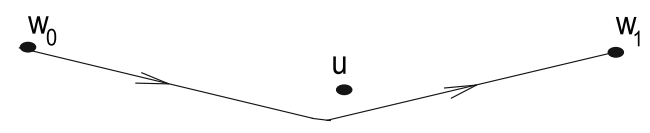

$$
\left[w_{0}, u^{-}, w_{1}\right]
$$

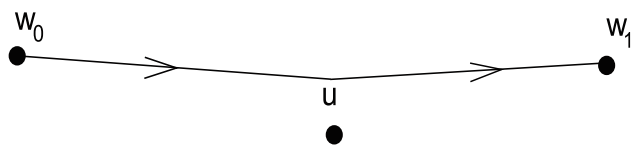

We may want to bypass a group of points, say $u_{1}, u_{2}$. Such contours are denoted by

$$
\left[w_{0},\left(u_{0}, u_{1}\right)^{+}, w_{1}\right],
$$

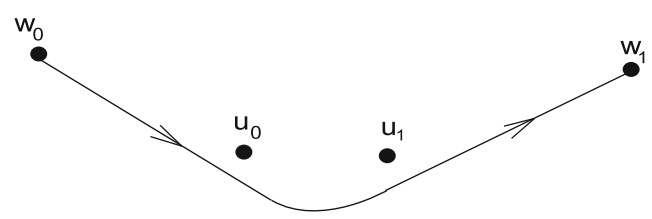




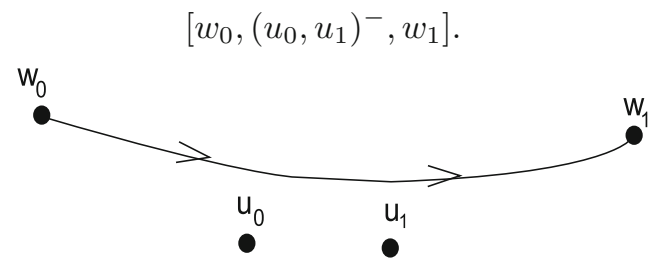

A small counterclockwise/clockwise loop around $u$ is denoted

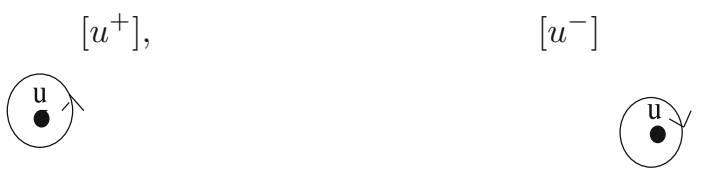

A counterclockwise/clockwise loop around a group of points, say, $u_{1}, u_{2}$ is denoted

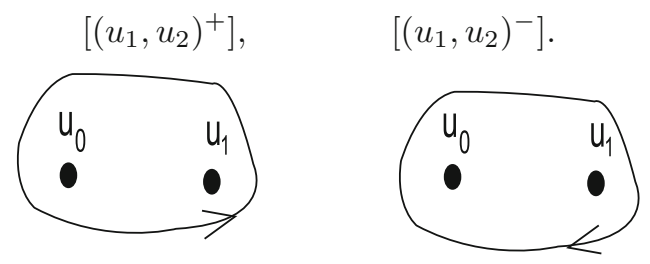

A half-line starting at $u$ and inclined at the angle $\phi$ is denoted $\left[u, \mathrm{e}^{\mathrm{i} \phi} \infty\left[:=\left\{u+\mathrm{e}^{\mathrm{i} \phi} t: t>0\right\}:\right.\right.$

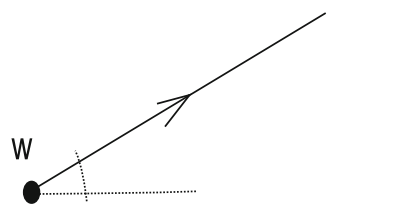

We will also need slightly more complicated contours:

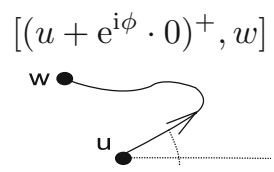

Here, the contour departs from $u$ at the angle $\phi$, then it bypasses $u$ with a small arc counterclockwise and then it goes in the direction of $w$.

The following contour has the shape of a kidney:

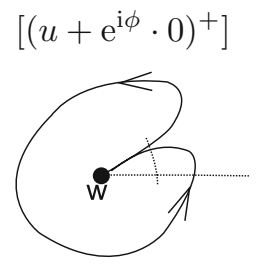


This contour departs from $u$ at the angle $\phi$, then it goes around $u$ and returns to $u$ again at the angle $\phi$.

Instead of $u+\mathrm{e}^{\mathrm{i} 0} \cdot 0$, we will write $u+0$. Likewise, instead of $u+\mathrm{e}^{\mathrm{i} \pi} \cdot 0$, we will write $u-0$.

\subsection{Reflection Invariant Differential Equations}

Consider a 2nd order differential operator

$$
\partial_{z}^{2}+b(z) \partial_{z}+c(z)
$$

Assume that (2.7) is invariant w.r.t. the reflection $z \mapsto-z$. This means that for some functions $\pi, \rho$, we have

$$
b(z)=z \pi\left(z^{2}\right), \quad c(z)=\rho\left(z^{2}\right) .
$$

Then it is natural to make a quadratic change of coordinates:

$$
\begin{gathered}
\partial_{z}^{2}+b(z) \partial_{z}+c(z)=4 u\left(\partial_{u}^{2}+\left(\frac{1}{2 u}+\frac{\pi(u)}{2}\right) \partial_{u}+\frac{\rho(u)}{4 u}\right), \\
z^{-1}\left(\partial_{z}^{2}+b(z) \partial_{z}+c(z)\right) z=4 u\left(\partial_{u}^{2}+\left(\frac{3}{2 u}+\frac{\pi(u)}{2}\right) \partial_{u}+\frac{\pi(u)+\rho(u)}{4 u}\right)
\end{gathered}
$$

where

$$
u=z^{2}, \quad z=\sqrt{u} .
$$

Thus, if $g_{+}(u)$, resp. $g_{-}(u)$ satisfy

$$
\begin{array}{r}
\left(\partial_{u}^{2}+\left(\frac{1}{2 u}+\frac{\pi(u)}{2}\right) \partial_{u}+\frac{\rho(u)}{4 u}\right) g_{+}(u)=0 \\
\left(\partial_{u}^{2}+\left(\frac{3}{2 u}+\frac{\pi(u)}{2}\right) \partial_{u}+\frac{\pi(u)+\rho(u)}{4 u}\right) g_{-}(u)=0
\end{array}
$$

then $g_{+}\left(z^{2}\right)$ is an even solution, resp. $z g_{-}\left(z^{2}\right)$ is an odd solution of the equation given by (2.7).

Note that if $\pi, \rho$ are holomorphic, then 0 is a regular singular point of (2.8) with indices $0, \frac{1}{2}$ and of (2.9) with indices $0,-\frac{1}{2}$.

\subsection{Regular Singular Points}

In this subsection, we recall well-known facts about regular singular points of differential equations

We will write

$$
f(z) \sim\left(z-z_{0}\right)^{\lambda} \text { at } z_{0}
$$

if $f(z)\left(z-z_{0}\right)^{-\lambda}$ is analytic at $z_{0}$ and $\lim _{z \rightarrow z_{0}} f(z)\left(z-z_{0}\right)^{-\lambda}=1$. In particular, we write

$$
f(z) \sim 1 \text { at } z_{0}
$$

if $f$ is analytic in a neighborhood of $z_{0}$ and $f\left(z_{0}\right)=1$. 
An equation given by the operator

$$
\partial_{z}^{2}+b(z) \partial_{z}+c(z)
$$

with meromorphic coefficients $a(z), c(z)$ has a regular singular point at $z_{0}$ if

$$
b_{0}:=\lim _{z \rightarrow z_{0}} b(z)\left(z-z_{0}\right), \quad c_{0}:=\lim _{z \rightarrow z_{0}} c(z)\left(z-z_{0}\right)^{2}
$$

exist. The indices $\lambda_{1}, \lambda_{2}$ of $z_{0}$ are the solutions of the indicial equation

$$
\lambda(\lambda-1)+b_{0} \lambda+c_{0}=0 .
$$

Theorem 2.1 (The Frobenius method). If $\lambda_{1}-\lambda_{2} \neq-1,-2, \ldots$, then there exists a unique solution $f(z)$ of the equation given by (2.10) such that $f(z) \sim$ $\left(z-z_{0}\right)^{\lambda_{1}}$ at $z_{0}$.

The case $\lambda_{1}-\lambda_{2} \in \mathbb{Z}$ is called the degenerate case. In this case, the Frobenius method gives one solution corresponding to the point $z_{0}$.

Likewise, (2.10) has a regular singular point at $\infty$ if

$$
\tilde{b}_{0}:=\lim _{z \rightarrow \infty} b(z) z, \quad \tilde{c}_{0}:=\lim _{z \rightarrow \infty} c(z) z^{2}
$$

exist. The indices $\tilde{\lambda}_{1}, \tilde{\lambda}_{2}$ of $\infty$ are the solutions of the indicial equation

$$
\tilde{\lambda}(\tilde{\lambda}+1)-\tilde{b}_{0} \tilde{\lambda}+\tilde{c}_{0}=0 .
$$

Theorem 2.2 (The Frobenius method at infinity). If $-\tilde{\lambda}_{1}+\tilde{\lambda}_{2} \neq-1,-2, \ldots$, then there exists a unique solution $\tilde{f}_{1}(z)$ of $(2.10)$ such that $\tilde{f}_{1}(z) \sim z^{-\tilde{\lambda}_{1}}$ at $\infty$.

Note the identity

$$
\begin{aligned}
& \left(z-z_{0}\right)^{-\theta}\left(\partial_{z}^{2}+b(z) \partial_{z}+c(z)\right)\left(z-z_{0}\right)^{\theta} \\
& \quad=\partial_{z}^{2}+\left(2 \theta\left(z-z_{0}\right)^{-1}+b(z)\right) \partial_{z}+\left(\theta^{2}-\theta\right)\left(z-z_{0}\right)^{-2}+\theta b(z)\left(z-z_{0}\right)^{-1}+c(z) .
\end{aligned}
$$

If $z_{0}$ is a regular singular point, then the corresponding indices of (2.11) equal those of $(2.10)+\theta$. Likewise, if $\infty$ is a regular singular point, then the corresponding indices are shifted by $-\theta$. The indices corresponding to other points are left unchanged.

\subsection{The Gamma Function}

In this section, we collect basic identities related to Euler's Gamma function that we will use.

$$
\begin{aligned}
\text { Relationship to factorial } & \Gamma(n+1)=n !, \quad n=0,1,2, \ldots, \\
\text { Recurrence relation } & \Gamma(z+1)=z \Gamma(z),
\end{aligned}
$$


Reflection formula $\Gamma(z) \Gamma(1-z)=\frac{\pi}{\sin \pi z}$,

II Euler's integral. $\Gamma(z):=\int_{0}^{\infty} \mathrm{e}^{-t} t^{z-1} \mathrm{~d} t, \quad \operatorname{Re} z>0$

Hankel's formula. $\frac{1}{\Gamma(-z+1)}=\frac{1}{2 \pi \mathrm{i}} \int_{\left[-\infty, 0^{+},-\infty[\right.} \mathrm{e}^{t} t^{z-1} \mathrm{~d} t$,

Legendre's formula $2^{2 z-1} \Gamma(z) \Gamma(z+1 / 2)=\sqrt{\pi} \Gamma(2 z)$.

I Euler's integral and its consequences.

$$
\begin{aligned}
& \frac{\Gamma(u) \Gamma(v)}{\Gamma(u+v)}=\int_{0}^{1} t^{u-1}(1-t)^{v-1} \mathrm{~d} t \quad \operatorname{Re} u>0, \operatorname{Re} v>0 \\
& \frac{\Gamma(u) \Gamma(v)}{\Gamma(u+v)} \frac{\sin \pi u}{\sin \pi(u+v)}=\frac{\Gamma(1-u-v) \Gamma(v)}{\Gamma(1-u)} \\
& =\int_{1}^{\infty} t^{u-1}(t-1)^{v-1} \mathrm{~d} t \quad \operatorname{Re} v>0, \operatorname{Re}(1-u-v)>0, \\
& \frac{\Gamma(-u) \Gamma(-v)}{\Gamma(-u-v)} \frac{\sin \pi u}{\sin \pi(u+v)}=\frac{\Gamma(-u-v+1)}{\Gamma(-u+1) \Gamma(-v+1)} \\
& =\frac{1}{2 \pi \mathrm{i}} \int_{]-\infty, 0^{+},-\infty[} t^{u-1}(1-t)^{v-1} \mathrm{~d} t \\
& =\frac{1}{2 \pi \mathrm{i}} \int_{] \infty, 1^{-}, \infty[} t^{u-1}(1-t)^{v-1} \mathrm{~d} t, \quad \operatorname{Re}(-u-v+1)>0 . \\
& \frac{\Gamma(u) \Gamma(v)}{\Gamma(u+v)} \sin \pi u=\frac{\Gamma(v)}{\Gamma(1-u) \Gamma(u+v)} \\
& =\frac{1}{2 \pi \mathrm{i}} \int_{] 1,0^{+}, 1\right]} t^{u-1}(1-t)^{v-1} \mathrm{~d} t, \quad \operatorname{Re} v>0 . \\
& \frac{\Gamma(u) \sqrt{\pi}}{\Gamma\left(u+\frac{1}{2}\right)}=\int_{-1}^{1}\left(1-s^{2}\right)^{u-1} \mathrm{~d} s \\
& \frac{\Gamma(u) \sqrt{\pi}}{2 \cos \pi u \Gamma\left(u+\frac{1}{2}\right)}=\int_{1}^{\infty}\left(s^{2}-1\right)^{u-1} \mathrm{~d} s .
\end{aligned}
$$




\subsection{The Pochhammer Symbol}

If $a \in \mathbb{C}$ and $n \in \mathbb{Z}$, then the so-called Pochhammer symbol is defined as follows:

$$
\begin{array}{ll}
(a)_{0}=1 \\
(a)_{n}:=a(a+1) \ldots(a+n-1), & n=1,2, \ldots \\
(a)_{n}:=\frac{1}{(a-n) \ldots(a-1)}, & n=\ldots,-2,-1 .
\end{array}
$$

Note the identities

$$
\begin{aligned}
(a)_{n} & =\frac{\Gamma(a+n)}{\Gamma(a)}=(-1)^{n} \frac{\Gamma(1-a)}{\Gamma(1-a-n)}=(-1)^{n}(1-n-a)_{n} \\
(1-z)^{-a} & =\sum_{n=0}^{\infty} \frac{(a)_{n}}{n !} z^{n}, \quad|z|<1 \\
(1 / 2)_{n} n ! & =\frac{(2 n) !}{2^{2 n}}, \quad(3 / 2)_{n} n !=\frac{(2 n+1) !}{2^{2 n}}
\end{aligned}
$$

\section{The ${ }_{2} F_{1}$ or the Hypergeometric Equation}

\subsection{Introduction}

Let $a, b, c \in \mathbb{C}$. Traditionally, the hypergeometric equation is given by the operator

$$
\mathcal{F}\left(a, b ; c ; z, \partial_{z}\right):=z(1-z) \partial_{z}^{2}+(c-(a+b+1) z) \partial_{z}-a b
$$

The classical parameters $a, b, c$ will be often replaced by another set of parameters $\alpha, \beta, \mu \in \mathbb{C}$, called Lie-algebraic. They are related to one another by

$$
\begin{aligned}
& \alpha:=c-1, \quad \beta:=a+b-c, \quad \mu:=b-a ; \\
& a=\frac{1+\alpha+\beta-\mu}{2}, \quad b=\frac{1+\alpha+\beta+\mu}{2}, \quad c=1+\alpha \text {. }
\end{aligned}
$$

In the Lie-algebraic parameters, the hypergeometric operator (3.1) becomes

$$
\begin{aligned}
& \mathcal{F}_{\alpha, \beta, \mu}\left(z, \partial_{z}\right) \\
& \quad=z(1-z) \partial_{z}^{2}+((1+\alpha)(1-z)-(1+\beta) z) \partial_{z}+\frac{1}{4} \mu^{2}-\frac{1}{4}(\alpha+\beta+1)^{2}
\end{aligned}
$$

The Lie-algebraic parameters have an interesting interpretation in terms of the natural basis of the Cartan algebra of the Lie algebra so(6) [2].

The singular points of the hypergeometric operator are located at $0,1, \infty$. All of them are regular singular. The indices of these points are 


\begin{tabular}{|l|l|l|}
\hline$z=0$ & $z=1$ & $z=\infty$ \\
\hline $1-c=-\alpha$ & $c-a-b=-\beta$ & $a=\frac{1+\alpha+\beta-\mu}{2}$ \\
0 & 0 & $b=\frac{1+\alpha+\beta+\mu}{2}$ \\
\hline
\end{tabular}

Thus, the Lie-algebraic parameters are the differences of the indices.

The hypergeometric operator remains the same if we interchange $a$ and $b$ (replace $\mu$ with $-\mu$ ).

\subsection{Integral Representations}

Theorem 3.1. Let $[0,1] \ni t \mapsto \gamma(t)$ satisfy

$$
\left.t^{b-c+1}(1-t)^{c-a}(t-z)^{-b-1}\right|_{\gamma(0)} ^{\gamma(1)}=0 .
$$

Then

$$
\mathcal{F}\left(a, b ; c ; z, \partial_{z}\right) \int_{\gamma} t^{b-c}(1-t)^{c-a-1}(t-z)^{-b} \mathrm{~d} t=0
$$

Proof. We check that for any contour $\gamma(3.3)$ equals

$$
-b \int_{\gamma}\left(\partial_{t} t^{b-c+1}(1-t)^{c-a}(t-z)^{-b-1}\right) \mathrm{d} t .
$$

Analogous (and nonequivalent) integral representations can be obtained by interchanging $a$ and $b$ in Theorem 3.1 .

\subsection{Symmetries}

To every permutation of the set of singularities $\{0,1, \infty\}$, we can associate exactly one homography $z \mapsto w(z)$. Using the method described at the end of Sect. 2.5, with every such homography we can associate 4 substitutions that preserve the form of the hypergeometric equation. Altogether there are $6 \times 4=24$ substitutions. They form a group isomorphic to the group of proper symmetries of the cube. If we take into account the fact that replacing $\mu$ with $-\mu$ is also an obvious symmetry of the hypergeometric equation, then we obtain a group of $2 \times 24=48$ elements, isomorphic to the group of all (proper and improper) symmetries of a cube, which is the Weyl group of $s o(6)$.

Below, we describe the table of symmetries of the hypergeometric operator except for those obtained by switching the sign of the last parameter. We fix the sign of the last parameter by demanding that the number of minus signs is even.

Note that the table looks much simpler in the Lie-algebraic parameters than in the classical parameters. 
All the operators below equal $\mathcal{F}_{\alpha, \beta, \mu}\left(w, \partial_{w}\right)$ for the corresponding $w$ : $w=z$ :

$w=1-z:$

$$
\begin{array}{rll} 
& \mathcal{F}_{\alpha, \beta, \mu}\left(z, \partial_{z}\right), & \\
(-z)^{-\alpha}(z-1)^{-\beta} & \mathcal{F}_{-\alpha,-\beta, \mu}\left(z, \partial_{z}\right) & (-z)^{\alpha}(z-1)^{\beta} \\
(z-1)^{-\beta} & \mathcal{F}_{\alpha,-\beta,-\mu}\left(z, \partial_{z}\right) & (z-1)^{\beta} \\
(-z)^{-\alpha} & \mathcal{F}_{-\alpha, \beta,-\mu}\left(z, \partial_{z}\right) & (-z)^{\alpha}
\end{array}
$$

$$
\begin{aligned}
& \mathcal{F}_{\beta, \alpha, \mu}\left(z, \partial_{z}\right) \\
& (z-1)^{-\alpha}(-z)^{-\beta} \quad \mathcal{F}_{-\beta,-\alpha, \mu}\left(z, \partial_{z}\right) \quad(z-1)^{\alpha}(-z)^{\beta}, \\
& (z-1)^{-\alpha} \quad \mathcal{F}_{\beta,-\alpha,-\mu}\left(z, \partial_{z}\right) \quad(z-1)^{\alpha}, \\
& (-z)^{-\beta} \quad \mathcal{F}_{-\beta, \alpha,-\mu}\left(z, \partial_{z}\right) \quad(-z)^{\beta} \\
& w=\frac{1}{z}: \\
& w=1-\frac{1}{z}: \\
& (-z)^{\frac{1}{2}(\alpha+\beta+\mu+1)} \quad(-z) \mathcal{F}_{\mu, \beta, \alpha}\left(z, \partial_{z}\right) \quad(-z)^{\frac{1}{2}(-\alpha-\beta-\mu-1)}, \\
& (-z)^{\frac{1}{2}(\alpha+\beta-\mu+1)}(z-1)^{-\beta} \quad(-z) \mathcal{F}_{-\mu,-\beta, \alpha}\left(z, \partial_{z}\right) \quad(-z)^{\frac{1}{2}(-\alpha-\beta+\mu-1)}(z-1)^{\beta}, \\
& (-z)^{\frac{1}{2}(\alpha+\beta+\mu+1)}(z-1)^{-\beta} \quad(-z) \mathcal{F}_{\mu,-\beta,-\alpha}\left(z, \partial_{z}\right) \quad(-z)^{\frac{1}{2}(-\alpha-\beta-\mu-1)}(z-1)^{\beta}, \\
& (-z)^{\frac{1}{2}(\alpha+\beta-\mu+1)} \quad(-z) \mathcal{F}_{-\mu, \beta,-\alpha}\left(z, \partial_{z}\right) \quad(-z)^{\frac{1}{2}(-\alpha-\beta+\mu-1)} ; \\
& w=\frac{1}{1-z}: \\
& (-z)^{\frac{1}{2}(\alpha+\beta+\mu+1)} \quad(-z) \mathcal{F}_{\mu, \alpha, \beta}\left(z, \partial_{z}\right) \quad(-z)^{\frac{1}{2}(-\alpha-\beta-\mu-1)}, \\
& (-z)^{\frac{1}{2}(\alpha+\beta-\mu+1)}(z-1)^{-\alpha} \quad(-z) \mathcal{F}_{-\mu,-\alpha, \beta}\left(z, \partial_{z}\right) \quad(-z)^{\frac{1}{2}(-\alpha-\beta+\mu-1)}(z-1)^{\alpha}, \\
& (-z)^{\frac{1}{2}(\alpha+\beta+\mu+1)}(z-1)^{-\alpha} \quad(-z) \mathcal{F}_{\mu,-\alpha,-\beta}\left(z, \partial_{z}\right) \quad(-z)^{\frac{1}{2}(-\alpha-\beta-\mu-1)}(z-1)^{\alpha} \text {, } \\
& (-z)^{\frac{1}{2}(\alpha+\beta-\mu+1)} \quad(-z) \mathcal{F}_{-\mu, \alpha,-\beta}\left(z, \partial_{z}\right) \quad(-z)^{\frac{1}{2}(-\alpha-\beta+\mu-1)} ; \\
& (z-1)^{\frac{1}{2}(\alpha+\beta+\mu+1)} \quad(z-1) \mathcal{F}_{\beta, \mu, \alpha}\left(z, \partial_{z}\right) \quad(z-1)^{\frac{1}{2}(-\alpha-\beta-\mu-1)}, \\
& (-z)^{-\beta}(z-1)^{\frac{1}{2}(\alpha+\beta-\mu+1)} \quad(z-1) \mathcal{F}_{-\beta,-\mu, \alpha}\left(z, \partial_{z}\right) \quad(-z)^{\beta}(z-1)^{\frac{1}{2}(-\alpha-\beta+\mu-1)}, \\
& (z-1)^{\frac{1}{2}(\alpha+\beta-\mu+1)} \quad(z-1) \mathcal{F}_{\beta,-\mu,-\alpha}\left(z, \partial_{z}\right) \quad(z-1)^{\frac{1}{2}(-\alpha-\beta+\mu-1)}, \\
& (-z)^{-\beta}(z-1)^{\frac{1}{2}(\alpha+\beta+\mu+1)} \quad(z-1) \mathcal{F}_{-\beta, \mu,-\alpha}\left(z, \partial_{z}\right) \quad(-z)^{\beta}(z-1)^{\frac{1}{2}(-\alpha-\beta-\mu-1)} ; \\
& (z-1)^{\frac{1}{2}(\alpha+\beta+\mu+1)} \quad(z-1) \mathcal{F}_{\alpha, \mu, \beta}\left(z, \partial_{z}\right) \quad(z-1)^{\frac{1}{2}(-\alpha-\beta-\mu-1)}, \\
& (-z)^{-\alpha}(z-1)^{\frac{1}{2}(\alpha+\beta-\mu+1)} \quad(z-1) \mathcal{F}_{-\alpha,-\mu, \beta}\left(z, \partial_{z}\right) \quad(-z)^{\alpha}(z-1)^{\frac{1}{2}(-\alpha-\beta+\mu-1)}, \\
& (z-1)^{\frac{1}{2}(\alpha+\beta-\mu+1)} \quad(z-1) \mathcal{F}_{\alpha,-\mu,-\beta}\left(z, \partial_{z}\right) \quad(z-1)^{\frac{1}{2}(-\alpha-\beta+\mu-1)}, \\
& (-z)^{-\alpha}(z-1)^{\frac{1}{2}(\alpha+\beta+\mu+1)} \quad(z-1) \mathcal{F}_{-\alpha, \mu,-\beta}\left(z, \partial_{z}\right) \quad(-z)^{\alpha}(z-1)^{\frac{1}{2}(-\alpha-\beta-\mu-1)} \text {. }
\end{aligned}
$$

\subsection{Factorization and Commutation Relations}

The hypergeometric operator can be factorized in several ways:

$$
\begin{aligned}
\mathcal{F}_{\alpha, \beta, \mu}= & \left(z(1-z) \partial_{z}+((1+\alpha)(1-z)-(1+\beta) z)\right) \partial_{z} \\
& -\frac{1}{4}(\alpha+\beta+\mu+1)(\alpha+\beta-\mu+1), \\
= & \partial_{z}\left(z(1-z) \partial_{z}+(\alpha(1-z)-\beta z)\right) \\
& -\frac{1}{4}(\alpha+\beta+\mu-1)(\alpha+\beta-\mu-1),
\end{aligned}
$$




$$
\begin{aligned}
= & \left((1-z) \partial_{z}-\beta-1\right)\left(z \partial_{z}+\alpha\right) \\
& -\frac{1}{4}(\alpha+\beta+\mu+1)(\alpha+\beta-\mu+1), \\
= & \left(z \partial_{z}+\alpha+1\right)\left((1-z) \partial_{z}-\beta\right) \\
& -\frac{1}{4}(\alpha+\beta+\mu+1)(\alpha+\beta-\mu+1) ;
\end{aligned}
$$

$$
\begin{aligned}
& z \mathcal{F}_{\alpha, \beta, \mu} \\
& =\left(z \partial_{z}+\frac{1}{2}(\alpha+\beta+\mu-1)\right)\left(z(1-z) \partial_{z}+\frac{1}{2}(1-z)(\alpha+\beta-\mu+1)-\beta\right) \\
& -\frac{1}{4}(\alpha+\beta+\mu-1)(\alpha-\beta-\mu+1), \\
& =\left(z(1-z) \partial_{z}+\frac{1}{2}(1-z)(\alpha+\beta-\mu+1)-\beta-1\right) \\
& \times\left(z \partial_{z}+\frac{1}{2}(\alpha+\beta+\mu+1)\right) \\
& -\frac{1}{4}(\alpha+\beta+\mu+1)(\alpha-\beta-\mu-1), \\
& =\left(z \partial_{z}+\frac{1}{2}(\alpha+\beta-\mu-1)\right)\left(z(1-z) \partial_{z}+\frac{1}{2}(1-z)(\alpha+\beta+\mu+1)-\beta\right) \\
& -\frac{1}{4}(\alpha+\beta-\mu-1)(\alpha-\beta+\mu+1) \\
& =\left(z(1-z) \partial_{z}+\frac{1}{2}(1-z)(\alpha+\beta+\mu+1)-\beta-1\right) \\
& \times\left(z \partial_{z}+\frac{1}{2}(\alpha+\beta-\mu+1)\right) \\
& -\frac{1}{4}(\alpha+\beta-\mu+1)(\alpha-\beta+\mu-1) \\
& (z-1) \mathcal{F}_{\alpha, \beta, \mu} \\
& =\left((z-1) \partial_{z}+\frac{1}{2}(\alpha+\beta+\mu-1)\right)\left(z(1-z) \partial_{z}+\frac{1}{2} z(-\alpha-\beta+\mu-1)+\alpha\right) \\
& -\frac{1}{4}(\alpha+\beta+\mu-1)(\alpha-\beta+\mu-1), \\
& =\left(z(1-z) \partial_{z}+\frac{1}{2} z(-\alpha-\beta+\mu-1)+\alpha+1\right) \\
& \times\left((z-1) \partial_{z}+\frac{1}{2}(\alpha+\beta+\mu+1)\right) \\
& -\frac{1}{4}(\alpha+\beta+\mu+1)(\alpha-\beta+\mu+1) \text {, } \\
& =\left((z-1) \partial_{z}+\frac{1}{2}(\alpha+\beta-\mu-1)\right)\left(z(1-z) \partial_{z}+\frac{1}{2} z(-\alpha-\beta-\mu-1)+\alpha\right) \\
& -\frac{1}{4}(\alpha+\beta-\mu-1)(\alpha-\beta-\mu-1),
\end{aligned}
$$




$$
\begin{aligned}
= & \left(z(1-z) \partial_{z}+\frac{1}{2} z(-\alpha-\beta-\mu-1)+\alpha+1\right) \\
& \times\left((z-1) \partial_{z}+\frac{1}{2}(\alpha+\beta-\mu+1)\right) \\
& -\frac{1}{4}(\alpha+\beta-\mu+1)(\alpha-\beta-\mu+1) .
\end{aligned}
$$

One way of showing the above factorizations is as follows: We start with deriving the first one, and then we apply the symmetries of Sect. 3.3.

The factorizations can be used to derive the following commutation relations:

$$
\begin{aligned}
& \partial_{z} \quad \mathcal{F}_{\alpha, \beta, \mu} \\
& =\mathcal{F}_{\alpha+1, \beta+1, \mu} \quad \partial_{z}, \\
& \left(z(1-z) \partial_{z}+(1-z) \alpha-z \beta\right) \quad \mathcal{F}_{\alpha, \beta, \mu} \\
& =\mathcal{F}_{\alpha-1, \beta-1, \mu} \quad\left(z(1-z) \partial_{z}+(1-z) \alpha-z \beta\right), \\
& \left((1-z) \partial_{z}-\beta\right) \quad \mathcal{F}_{\alpha, \beta, \mu} \\
& =\mathcal{F}_{\alpha+1, \beta-1, \mu} \quad\left((1-z) \partial_{z}-\beta\right), \\
& \left(z \partial_{z}+\alpha\right) \quad \mathcal{F}_{\alpha, \beta, \mu} \\
& =\mathcal{F}_{\alpha-1, \beta+1, \mu} \quad\left(z \partial_{z}+\alpha\right) ; \\
& \left(z \partial_{z}+\frac{1}{2}(\alpha+\beta+\mu+1)\right) \quad z \mathcal{F}_{\alpha, \beta, \mu} \\
& =z \mathcal{F}_{\alpha, \beta+1, \mu+1} \quad\left(z \partial_{z}+\frac{1}{2}(\alpha+\beta+\mu+1)\right), \\
& \left(z(1-z) \partial_{z}+\frac{1}{2}(1-z)(\alpha+\beta-\mu+1)-\beta\right) \quad z \mathcal{F}_{\alpha, \beta, \mu} \\
& =z \mathcal{F}_{\alpha, \beta-1, \mu-1} \quad\left(z(1-z) \partial_{z}+\frac{1}{2}(1-z)(\alpha+\beta-\mu+1)-\beta\right), \\
& \left(z \partial_{z}+\frac{1}{2}(\alpha+\beta-\mu+1)\right) \quad z \mathcal{F}_{\alpha, \beta, \mu} \\
& =z \mathcal{F}_{\alpha, \beta+1, \mu-1} \quad\left(z \partial_{z}+\frac{1}{2}(\alpha+\beta-\mu+1),\right. \\
& \left(z(z-1) \partial_{z}-\frac{1}{2}(1-z)(\alpha+\beta+\mu+1)+\beta\right) \quad z \mathcal{F}_{\alpha, \beta, \mu} \\
& =z \mathcal{F}_{\alpha, \beta-1, \mu+1} \quad\left(z(z-1) \partial_{z}-\frac{1}{2}(1-z)(\alpha+\beta+\mu+1)+\beta\right) ; \\
& \left((z-1) \partial_{z}+\frac{1}{2}(\alpha+\beta+\mu+1)\right) \quad(1-z) \mathcal{F}_{\alpha, \beta, \mu} \\
& =(1-z) \mathcal{F}_{\alpha+1, \beta, \mu+1} \quad\left((z-1) \partial_{z}+\frac{1}{2}(\alpha+\beta+\mu+1),\right. \\
& \left(z(1-z) \partial_{z}-\frac{1}{2} z(\alpha+\beta-\mu+1)+\alpha\right) \quad(1-z) \mathcal{F}_{\alpha, \beta, \mu} \\
& =(1-z) \mathcal{F}_{\alpha-1, \beta, \mu-1} \quad\left(z(1-z) \partial_{z}-\frac{1}{2} z(\alpha+\beta-\mu+1)+\alpha\right), \\
& \left((z-1) \partial_{z}+\frac{1}{2}(\alpha+\beta-\mu+1)\right) \quad(1-z) \mathcal{F}_{\alpha, \beta, \mu} \\
& =(1-z) \mathcal{F}_{\alpha+1, \beta, \mu-1} \quad\left((z-1) \partial_{z}+\frac{1}{2}(\alpha+\beta-\mu+1)\right), \\
& \left(z(z-1) \partial_{z}+\frac{1}{2} z(\alpha+\beta+\mu+1)-\alpha\right) \quad(1-z) \mathcal{F}_{\alpha, \beta, \mu} \\
& =(1-z) \mathcal{F}_{\alpha-1, \beta, \mu+1} \quad\left(z(z-1) \partial_{z}+\frac{1}{2} z(\alpha+\beta+\mu+1)-\alpha\right) .
\end{aligned}
$$

Each of these commutation relations corresponds to a root of the Lie algebra so(6).

\subsection{Canonical Forms}

The natural weight of the hypergeometric operator is $z^{\alpha}(1-z)^{\beta}$, so that

$$
\mathcal{F}_{\alpha, \beta, \mu}=z^{-\alpha}(1-z)^{-\beta} \partial_{z} z^{\alpha+1}(1-z)^{\beta+1} \partial_{z}+\frac{\mu^{2}}{4}-\frac{(\alpha+\beta+1)^{2}}{4} .
$$


The balanced form of the hypergeometric operator is

$$
z^{\frac{\alpha}{2}}(1-z)^{\frac{\beta}{2}} \mathcal{F}_{\alpha, \beta, \mu} z^{-\frac{\alpha}{2}}(1-z)^{-\frac{\beta}{2}}=\partial_{z} z(1-z) \partial_{z}-\frac{\alpha^{2}}{4 z}-\frac{\beta^{2}}{4(1-z)}+\frac{\mu^{2}-1}{4} .
$$

Note that the symmetries $\alpha \rightarrow-\alpha, \beta \rightarrow-\beta$ and $\mu \rightarrow-\mu$ are obvious in the balanced form.

Remark 3.2. In the literature, the balanced form of the hypergeometric equation is sometimes called the generalized associated Legendre equation. Its standard form according to [12] is

$$
\left(1-w^{2}\right) \partial_{w}^{2}-2 w \partial_{w}+\nu(\nu+1)-\frac{\mu_{1}^{2}}{2(1-w)}-\frac{\mu_{1}^{2}}{2(1+w)} .
$$

Thus, $z=\frac{w+1}{2}$, moreover, $\mu_{1}, \mu_{2}$ and $\nu$ correspond to $\beta, \alpha$ and $\frac{\mu}{2}-\frac{1}{2}$.

\subsection{The Hypergeometric Function}

0 is a regular singular point of the hypergeometric equation. Its indices are 0 and $1-c$. The Frobenius method implies that, for $c \neq 0,-1,-2, \ldots$, the unique solution of the hypergeometric equation equal to 1 at 0 is given by the series

$$
F(a, b ; c ; z)=\sum_{j=0}^{\infty} \frac{(a)_{j}(b)_{j}}{(c)_{j}} \frac{z^{j}}{j !},
$$

convergent for $|z|<1$. The function extends to the whole complex plane cut at $[1, \infty[$ and is called the hypergeometric function. Sometimes, it is more convenient to consider the function

$$
\mathbf{F}(a, b ; c ; z):=\frac{F(a, b, c, z)}{\Gamma(c)}=\sum_{j=0}^{\infty} \frac{(a)_{j}(b)_{j}}{\Gamma(c+j)} \frac{z^{j}}{j !}
$$

defined for all $a, b, c \in \mathbb{C}$. Another useful function proportional to ${ }_{2} F_{1}$ is

$$
\mathbf{F}^{\mathrm{I}}(a, b ; c ; z):=\frac{\Gamma(a) \Gamma(c-a)}{\Gamma(c)} F(a, b ; c ; z)=\sum_{j=0}^{\infty} \frac{\Gamma(a+j) \Gamma(c-a)(b)_{j}}{\Gamma(c+j)} \frac{z^{j}}{j !} .
$$

It has the integral representation

$$
\begin{aligned}
& \int_{1}^{\infty} t^{b-c}(t-1)^{c-a-1}(t-z)^{-b} \mathrm{~d} t \\
& \quad=\mathbf{F}^{\mathrm{I}}(a, b ; c ; z), \quad \operatorname{Re}(c-a)>0, \operatorname{Re} a>0, \quad z \notin[1, \infty[.
\end{aligned}
$$

Indeed, by Theorem 3.1, the left hand side of (3.5) is annihilated by the hypergeometric operator (3.1). Besides, by (2.19) it equals $\frac{\Gamma(a) \Gamma(c-a)}{\Gamma(c)}$ at 0 . So does the right hand side. Therefore, Equation (3.5) follows by the uniqueness of the solution by the Frobenius method.

Another, closely related integral representation is

$$
\frac{\sin \pi a}{\pi} \mathbf{F}^{\mathrm{I}}(a, b ; c ; z)=\frac{1}{2 \pi \mathrm{i}} \int_{\left[1,(z, 0)^{+}, 1\right]}(-t)^{b-c}(1-t)^{c-a-1}(z-t)^{-b} \mathrm{~d} t .
$$


It is proven essentially in the same way as (3.5), except that instead of (2.19) we use (2.21). We have the identities

$$
\begin{aligned}
F(a, b ; c ; z) & =(1-z)^{c-a-b} F(c-a, c-b ; c ; z) \\
& =(1-z)^{-a} F\left(a, c-b ; c ; \frac{z}{z-1}\right) \\
& =(1-z)^{-b} F\left(c-a, b ; c ; \frac{z}{z-1}\right) .
\end{aligned}
$$

In fact, by the 3rd, 9th and 11th symmetry of Sect. 3.3, all these functions are annihilated by the hypergeometric operator. All of them are $\sim 1$ at 1 . Hence, by the uniqueness of the Frobenius method they coincide, at least for $c \neq 0,-1, \ldots$ By continuity, the identities hold for all $c \in \mathbb{C}$.

Let us introduce new notation for various varieties of the hypergeometric function involving the Lie-algebraic parameters instead of the classical parameters.

$$
\begin{aligned}
F_{\alpha, \beta, \mu}(z) & =F\left(\frac{1+\alpha+\beta-\mu}{2}, \frac{1+\alpha+\beta+\mu}{2} ; 1+\alpha ; z\right), \\
\mathbf{F}_{\alpha, \beta, \mu}(z) & =\mathbf{F}\left(\frac{1+\alpha+\beta-\mu}{2}, \frac{1+\alpha+\beta+\mu}{2} ; 1+\alpha ; z\right) \\
& =\frac{1}{\Gamma(\alpha+1)} F_{\alpha, \beta, \mu}(z), \\
\mathbf{F}_{\alpha, \beta, \mu}^{\mathrm{I}}(z) & =\mathbf{F}^{\mathrm{I}}\left(\frac{1+\alpha+\beta-\mu}{2}, \frac{1+\alpha+\beta+\mu}{2} ; 1+\alpha ; z\right) \\
& =\frac{\Gamma\left(\frac{1+\alpha+\beta-\mu}{2}\right) \Gamma\left(\frac{1+\alpha-\beta+\mu}{2}\right)}{\Gamma(\alpha+1)} F_{\alpha, \beta, \mu}(z) .
\end{aligned}
$$

\subsection{Standard Solutions: Kummer's Table}

To each of the singular points $0,1, \infty$, we can associate two solutions corresponding to its indices. Thus, we obtain $3 \times 2=6$ solutions, which we will call standard solutions. Using the identities (3.7), each solution can be written in 4 distinct ways (not counting the trivial change of the sign in front of the last parameter). Thus, we obtain a list of $6 \times 4=24$ expressions for solutions of the hypergeometric equation, called sometimes Kummer's table.

We describe the standard solutions to the hypergeometric equation in this section. We will use consistently the Lie-algebraic parameters, which give much simpler expressions.

It follows from Theorem 3.1 that for appropriate contours $\gamma$ integrals of the form

$$
\int_{\gamma} t^{\frac{-1-\alpha+\beta+\mu}{2}}(t-1)^{\frac{-1+\alpha-\beta+\mu}{2}}(t-z)^{\frac{-1-\alpha-\beta-\mu}{2}} \mathrm{~d} t
$$

are solutions of the hypergeometric equation. The integrand has four singularities: $\{0,1, \infty, z\}$. It is natural to chose $\gamma$ as the interval joining a pair of singularities. This choice leads to 6 standard solutions with the I-type normalization. 
3.7.1. Solution $\sim \mathbf{1}$ at $\mathbf{0}$. If $\alpha \neq-1,-2, \ldots$, then the following function is the unique solution $\sim 1$ at 0 :

$$
\begin{aligned}
F_{\alpha, \beta, \mu}(z) & =(1-z)^{-\beta} F_{\alpha,-\beta,-\mu}(z) \\
& =(1-z)^{\frac{-1-\alpha-\beta+\mu}{2}} F_{\alpha,-\mu,-\beta}\left(\frac{z}{z-1}\right) \\
& =(1-z)^{\frac{-1-\alpha-\beta-\mu}{2}} F_{\alpha, \mu, \beta}\left(\frac{z}{z-1}\right) .
\end{aligned}
$$

An integral representation for $\operatorname{Re}(1+\alpha)>|\operatorname{Re}(\beta-\mu)|$ :

$$
\int_{1}^{\infty} t^{\frac{-1-\alpha+\beta+\mu}{2}}(t-1)^{\frac{-1+\alpha-\beta+\mu}{2}}(t-z)^{\frac{-1-\alpha-\beta-\mu}{2}} \mathrm{~d} t=\mathbf{F}_{\alpha, \beta, \mu}^{\mathrm{I}}(z), z \notin[1, \infty[.
$$

Note that all the identities of this subsubsection are the transcriptions of identities of Sect. 3.6 to the Lie-algebraic parameters.

3.7.2. Solution $\sim \boldsymbol{z}^{-\boldsymbol{\alpha}}$ at $\mathbf{0}$. If $\alpha \neq 1,2, \ldots$, then the following function is the unique solution behaving as $z^{-\alpha}$ at 0 :

$$
\begin{aligned}
z^{-\alpha} F_{-\alpha, \beta,-\mu}(z) & =z^{-\alpha}(1-z)^{-\beta} F_{-\alpha,-\beta, \mu}(z) \\
& =z^{-\alpha}(1-z)^{\frac{-1+\alpha-\beta+\mu}{2}} F_{-\alpha,-\mu, \beta}\left(\frac{z}{z-1}\right) \\
& =z^{-\alpha}(1-z)^{\frac{-1+\alpha-\beta-\mu}{2}} F_{-\alpha, \mu,-\beta}\left(\frac{z}{z-1}\right) .
\end{aligned}
$$

Integral representations for $\operatorname{Re}(1-\alpha)>|\operatorname{Re}(\beta-\mu)|$ :

$$
\begin{aligned}
& \int_{0}^{z} t^{\frac{-1-\alpha+\beta+\mu}{2}}(1-t)^{\frac{-1+\alpha-\beta+\mu}{2}}(z-t)^{\frac{-1-\alpha-\beta-\mu}{2}} \mathrm{~d} t=z^{-\alpha} \mathbf{F}_{-\alpha, \beta,-\mu}^{\mathrm{I}}(z), \\
& z \notin]-\infty, 0] \cup[1, \infty[; \\
& \int_{z}^{0}(-t)^{\frac{-1-\alpha+\beta+\mu}{2}}(1-t)^{\frac{-1+\alpha-\beta+\mu}{2}}(t-z)^{\frac{-1-\alpha-\beta-\mu}{2}} \mathrm{~d} t=(-z)^{-\alpha} \mathbf{F}_{-\alpha, \beta,-\mu}^{\mathrm{I}}(z), \\
& z \notin[0, \infty[.
\end{aligned}
$$

To check these identities, we note first that the integrals are solutions of the hypergeometric equation. By substituting $t=z s$, we easily check that they have the correct behavior at zero.

Of course, it is elementary to pass from the first identity, which is adapted to the region on the right of the singularity $z=0$ to the second, adapted to the region on the left of the singularity. For convenience, we give both identities.

3.7.3. Solution $\sim \mathbf{1}$ at $\mathbf{1}$. If $\beta \neq-1,-2, \ldots$, then the following function is the unique solution $\sim 1$ at 1 : 


$$
\begin{aligned}
F_{\beta, \alpha, \mu}(1-z) & =z^{-\alpha} F_{\beta,-\alpha,-\mu}(1-z) \\
& =z^{\frac{-1-\alpha-\beta+\mu}{2}} F_{\beta,-\mu,-\alpha}\left(1-z^{-1}\right) \\
& =z^{\frac{-1-\alpha-\beta-\mu}{2}} F_{\beta, \mu, \alpha}\left(1-z^{-1}\right)
\end{aligned}
$$

Integral representation for $\operatorname{Re}(1+\beta)>|\operatorname{Re}(\alpha-\mu)|$ :

$$
\begin{array}{r}
\int_{-\infty}^{0}(-t)^{\frac{-1-\alpha+\beta+\mu}{2}}(1-t)^{\frac{-1+\alpha-\beta+\mu}{2}}(z-t)^{\frac{-1-\alpha-\beta-\mu}{2} \mathrm{~d} t}=\mathbf{F}_{\beta, \alpha, \mu}^{\mathrm{I}}(1-z), \\
z \notin]-\infty, 0] .
\end{array}
$$

3.7.4. Solution $\sim(\mathbf{1}-\boldsymbol{z})^{-\boldsymbol{\beta}}$ at $\mathbf{1}$. If $\beta \neq 1,2, \ldots$, then the following function is the unique solution of the hypergeometric equation $\sim(1-z)^{-\beta}$ at 1 :

$$
\begin{aligned}
(1 & -z)^{-\beta} F_{-\beta, \alpha,-\mu}(1-z) \\
& =z^{-\alpha}(1-z)^{-\beta} F_{-\beta,-\alpha, \mu}(1-z) \\
& =z^{\frac{-1-\alpha+\beta-\mu}{2}}(1-z)^{-\beta} F_{-\beta, \mu,-\alpha}\left(1-z^{-1}\right) \\
& =z^{\frac{-1-\alpha+\beta+\mu}{2}}(1-z)^{-\beta} F_{-\beta,-\mu, \alpha}\left(1-z^{-1}\right) .
\end{aligned}
$$

Integral representations for $\operatorname{Re}(1-\beta)>|\operatorname{Re}(\alpha+\mu)|$ :

$$
\begin{aligned}
& \int_{z}^{1} t^{\frac{-1-\alpha+\beta+\mu}{2}}(1-t)^{\frac{-1+\alpha-\beta+\mu}{2}}(t-z)^{\frac{-1-\alpha-\beta-\mu}{2}} \mathrm{~d} t \\
& \left.\left.\quad=(1-z)^{-\beta} \mathbf{F}_{-\beta, \alpha,-\mu}^{\mathrm{I}}(1-z), \quad z \notin\right]-\infty, 0\right] \cup[1, \infty[; \\
& \int_{1}^{z} t^{\frac{-1-\alpha+\beta+\mu}{2}}(t-1)^{\frac{-1+\alpha-\beta+\mu}{2}}(z-t)^{\frac{-1-\alpha-\beta-\mu}{2}} \mathrm{~d} t \\
& \left.\left.\quad=(z-1)^{-\beta} \mathbf{F}_{-\beta, \alpha,-\mu}^{\mathrm{I}}(1-z), \quad z \notin\right]-\infty, 1\right] .
\end{aligned}
$$

3.7.5. Solution $\sim z^{-a}$ at $\infty$. If $\mu \neq 1,2 \ldots$, then the following function is the unique solution of the hypergeometric equation $\sim(-z)^{-a}=(-z)^{\frac{-1-\alpha-\beta+\mu}{2}}$ at $\infty$ :

$$
\begin{aligned}
& (-z)^{\frac{-1-\alpha-\beta+\mu}{2}} F_{-\mu, \beta,-\alpha}\left(z^{-1}\right) \\
& \quad=(-z)^{\frac{-1-\alpha+\beta+\mu}{2}}(1-z)^{-\beta} F_{-\mu,-\beta, \alpha}\left(z^{-1}\right) \\
& \quad=(1-z)^{\frac{-1-\alpha-\beta+\mu}{2}} F_{-\mu, \alpha,-\beta}\left((1-z)^{-1}\right) \\
& \quad=(-z)^{-\alpha}(1-z)^{\frac{-1+\alpha-\beta+\mu}{2}} F_{-\mu,-\alpha, \beta}\left((1-z)^{-1}\right) .
\end{aligned}
$$

Integral representations for $\operatorname{Re}(1-\mu)>|\operatorname{Re}(\alpha+\beta)|$ :

$$
\begin{aligned}
& \int_{z}^{\infty} t^{\frac{-1-\alpha+\beta+\mu}{2}}(t-1)^{\frac{-1+\alpha-\beta+\mu}{2}}(t-z)^{\frac{-1-\alpha-\beta-\mu}{2}} \mathrm{~d} t \\
& \left.\left.=z^{\frac{-1-\alpha-\beta-\mu}{2}} \mathbf{F}_{-\mu, \beta,-\alpha}^{\mathrm{I}}\left(z^{-1}\right), \quad z \notin\right]-\infty, 1\right] ;
\end{aligned}
$$




$$
\begin{aligned}
& \int_{-\infty}^{z}(-t)^{\frac{-1-\alpha+\beta+\mu}{2}}(1-t)^{\frac{-1+\alpha-\beta+\mu}{2}}(z-t)^{\frac{-1-\alpha-\beta-\mu}{2}} \mathrm{~d} t \\
& \left.\left.=(-z)^{\frac{-1-\alpha-\beta-\mu}{2}} \mathbf{F}_{-\mu, \beta,-\alpha}^{\mathrm{I}}\left(z^{-1}\right), \quad z \notin\right] 0, \infty\right] .
\end{aligned}
$$

3.7.6. Solution $\sim z^{-b}$ at $\infty$. If $\mu \neq-1,-2, \ldots$, then the following function is the unique solution of the hypergeometric equation $\sim(-z)^{-b}=(-z)^{\frac{-1-\alpha-\beta-\mu}{2}}$ at $\infty$ :

$$
\begin{aligned}
& (-z)^{\frac{-1-\alpha-\beta-\mu}{2}} F_{\mu, \beta, \alpha}\left(z^{-1}\right) \\
& \quad=(-z)^{\frac{-1-\alpha+\beta-\mu}{2}}(1-z)^{-\beta} F_{\mu,-\beta,-\alpha}\left(z^{-1}\right) \\
& \quad=(1-z)^{\frac{-1-\alpha-\beta-\mu}{2}} F_{\mu, \alpha, \beta}\left((1-z)^{-1}\right) \\
& \quad=(-z)^{-\alpha}(1-z)^{\frac{-1+\alpha-\beta-\mu}{2}} F_{\mu,-\alpha,-\beta}\left((1-z)^{-1}\right)
\end{aligned}
$$

Integral representations for $\operatorname{Re}(1+\mu)>|\operatorname{Re}(\alpha-\beta)|$ :

$$
\begin{gathered}
\int_{0}^{1} t^{\frac{-1-\alpha+\beta-\mu}{2}}(1-t)^{\frac{-1+\alpha-\beta+\mu}{2}}(t-z)^{\frac{-1-\alpha-\beta-\mu}{2}} \mathrm{~d} t \\
=(-z)^{\frac{-1-\alpha-\beta+\mu}{2}} \mathbf{F}_{\mu, \beta, \alpha}^{\mathrm{I}}\left(z^{-1}\right), \quad z \notin[0, \infty[; \\
\int_{0}^{1} t^{\frac{-1-\alpha+\beta-\mu}{2}}(1-t)^{\frac{-1+\alpha-\beta+\mu}{2}}(z-t)^{\frac{-1-\alpha-\beta-\mu}{2}} \mathrm{~d} t \\
=z^{\frac{-1-\alpha-\beta+\mu}{2}} \mathbf{F}_{\mu, \beta, \alpha}^{\mathrm{I}}\left(z^{-1}\right), \quad z \notin[-\infty, 1[.
\end{gathered}
$$

\subsection{Connection Formulas}

We use the solutions $\sim 1$ and $\sim z^{-\alpha}$ at 0 as the basis. We show how the other solutions decompose in this basis.

For the first pair of relations, we assume that $z \notin]-\infty, 0] \cup[1, \infty[$ :

$$
\begin{aligned}
\mathbf{F}_{\beta, \alpha, \mu}(1-z)= & \frac{\pi}{\sin \pi(-\alpha) \Gamma\left(\frac{1-\alpha+\beta-\mu}{2}\right) \Gamma\left(\frac{1-\alpha+\beta+\mu}{2}\right)} \mathbf{F}_{\alpha, \beta, \mu}(z) \\
& +\frac{\pi}{\sin \pi \alpha \Gamma\left(\frac{1+\alpha+\beta-\mu}{2}\right) \Gamma\left(\frac{1+\alpha+\beta+\mu}{2}\right)} z^{-\alpha} \mathbf{F}_{-\alpha, \beta,-\mu}(z), \\
(1-z)^{-\beta} \mathbf{F}_{-\beta, \alpha,-\mu}(1-z)= & \frac{\pi}{\sin \pi(-\alpha) \Gamma\left(\frac{1-\alpha-\beta+\mu}{2}\right) \Gamma\left(\frac{1-\alpha-\beta-\mu}{2}\right)} \mathbf{F}_{\alpha, \beta, \mu}(z) \\
& +\frac{\pi}{\sin \pi \alpha \Gamma\left(\frac{1+\alpha-\beta+\mu}{2}\right) \Gamma\left(\frac{1+\alpha-\beta-\mu}{2}\right)} z^{-\alpha} \mathbf{F}_{-\alpha, \beta,-\mu}(z) .
\end{aligned}
$$


For the second pair, we assume that $z \notin[0, \infty[$

$$
\begin{aligned}
& (-z) \frac{\pi-\alpha-\beta+\mu}{2} \mathbf{F}_{-\mu, \beta,-\alpha}\left(z^{-1}\right)=\frac{\pi}{\sin \pi(-\alpha) \Gamma\left(\frac{1-\alpha-\beta-\mu}{2}\right) \Gamma\left(\frac{1-\alpha+\beta-\mu}{2}\right)} \mathbf{F}_{\alpha, \beta, \mu}(z) \\
& +\frac{\pi}{\sin \pi \alpha \Gamma\left(\frac{1+\alpha+\beta-\mu}{2}\right) \Gamma\left(\frac{1+\alpha-\beta-\mu}{2}\right)}(-z)^{-\alpha} \mathbf{F}_{-\alpha, \beta,-\mu}(z), \\
& (-z) \frac{\pi}{\frac{-1-\alpha-\beta-\mu}{2}} \mathbf{F}_{\mu, \beta, \alpha}\left(z^{-1}\right)=\frac{\pi}{\sin \pi(-\alpha) \Gamma\left(\frac{1-\alpha-\beta+\mu}{2}\right) \Gamma\left(\frac{1-\alpha+\beta+\mu}{2}\right)} \mathbf{F}_{\alpha, \beta, \mu}(z) \\
& +\frac{\pi}{\sin \pi \alpha \Gamma\left(\frac{1+\alpha+\beta+\mu}{2}\right) \Gamma\left(\frac{1+\alpha-\beta+\mu}{2}\right)}(-z)^{-\alpha} \mathbf{F}_{-\alpha, \beta,-\mu}(z) .
\end{aligned}
$$

The connection formulas are easily derived from the integral representations by looking at the behavior around 0 .

\subsection{Recurrence Relations}

The following recurrence relations follow easily from the commutation relations of Sect. 3.4:

$$
\begin{aligned}
& \partial_{z} \mathbf{F}_{\alpha, \beta, \mu}^{\mathrm{I}}(z)=\frac{1+\alpha+\beta+\mu}{2} \mathbf{F}_{\alpha+1, \beta+1, \mu}^{\mathrm{I}}(z), \\
& \left(z(1-z) \partial_{z}+\alpha(1-z)-\beta z\right) \mathbf{F}_{\alpha, \beta, \mu}^{\mathrm{I}}(z)=\frac{-1+\alpha+\beta+\mu}{2} \mathbf{F}_{\alpha-1, \beta-1, \mu}^{\mathrm{I}}(z), \\
& \left((1-z) \partial_{z}-\beta\right) \mathbf{F}_{\alpha, \beta, \mu}^{\mathrm{I}}(z)=\frac{1+\alpha-\beta-\mu}{2} \mathbf{F}_{\alpha+1, \beta-1, \mu}^{\mathrm{I}}(z), \\
& \left(z \partial_{z}+\alpha\right) \mathbf{F}_{\alpha, \beta, \mu}^{\mathrm{I}}(z)=\frac{1+\alpha-\beta+\mu}{2} \mathbf{F}_{\alpha-1, \beta+1, \mu}^{\mathrm{I}}(z), \\
& \left(z \partial_{z}+\frac{1+\alpha+\beta+\mu}{2}\right) \mathbf{F}_{\alpha, \beta, \mu}^{\mathrm{I}}(z)=\frac{1+\alpha+\beta+\mu}{2} \mathbf{F}_{\alpha, \beta+1, \mu+1}^{\mathrm{I}}(z), \\
& \left(z(1-z) \partial_{z}-\beta+\frac{1+\alpha+\beta-\mu}{2}(1-z)\right) \mathbf{F}_{\alpha, \beta, \mu}^{\mathrm{I}}(z)=\frac{1+\alpha-\beta-\mu}{2} \mathbf{F}_{\alpha, \beta-1, \mu-1}^{\mathrm{I}}(z), \\
& \left(z \partial_{z}+\frac{1+\alpha+\beta-\mu}{2}\right) \mathbf{F}_{\alpha, \beta, \mu}^{\mathrm{I}}(z)=\frac{1+\alpha+\beta-\mu}{2} \mathbf{F}_{\alpha, \beta+1, \mu-1}^{\mathrm{I}}(z), \\
& \left(z(1-z) \partial_{z}-\beta+\frac{1+\alpha+\beta+\mu}{2}(1-z)\right) \mathbf{F}_{\alpha, \beta, \mu}^{\mathrm{I}}(z)=\frac{1+\alpha-\beta+\mu}{2} \mathbf{F}_{\alpha, \beta-1, \mu+1}^{\mathrm{I}}(z), \\
& \left((z-1) \partial_{z}+\frac{1+\alpha+\beta+\mu}{2}\right) \mathbf{F}_{\alpha, \beta, \mu}^{\mathrm{I}}(z)=\frac{1+\alpha+\beta+\mu}{2} \mathbf{F}_{\alpha+1, \beta, \mu+1}^{\mathrm{I}}(z), \\
& \left(z(1-z) \partial_{z}+\alpha-\frac{1+\alpha+\beta-\mu}{2} z\right) \mathbf{F}_{\alpha, \beta, \mu}^{\mathrm{I}}(z)=\frac{-1+\alpha-\beta+\mu}{2} \mathbf{F}_{\alpha-1, \beta, \mu-1}^{\mathrm{I}}(z),
\end{aligned}
$$




$$
\begin{aligned}
& \left((z-1) \partial_{z}+\frac{1+\alpha+\beta-\mu}{2}\right) \mathbf{F}_{\alpha, \beta, \mu}^{\mathrm{I}}(z)=\frac{1+\alpha-\beta-\mu}{2} \mathbf{F}_{\alpha+1, \beta, \mu-1}^{\mathrm{I}}(z) \\
& \left(z(1-z) \partial_{z}+\alpha-\frac{1+\alpha+\beta+\mu}{2} z\right) \mathbf{F}_{\alpha, \beta, \mu}^{\mathrm{I}}(z)=\frac{-1+\alpha+\beta-\mu}{2} \mathbf{F}_{\alpha-1, \beta, \mu+1}^{\mathrm{I}}(z) .
\end{aligned}
$$

\subsection{Additional Recurrence Relations}

There exist other, more complicated recurrence relations for hypergeometric functions, for example

$$
\begin{aligned}
& \left(\frac{(1+\alpha+\beta+\mu)(-1-\alpha+\beta-\mu)}{4}\right. \\
& \left.\quad+\frac{(1+\alpha+\beta+\mu)(\mu+1)}{2} z-(1+\mu) z(1-z) \partial_{z}\right) \mathbf{F}_{\alpha, \beta, \mu} \\
& \quad=\frac{(1+\alpha+\beta+\mu)(-1-\alpha+\beta-\mu)}{4} \mathbf{F}_{\alpha, \beta, \mu+2}(z) \\
& \left(\frac{(1+\alpha+\beta-\mu)(-1-\alpha+\beta+\mu)}{4}\right. \\
& \left.\quad+\frac{(1+\alpha+\beta-\mu)(-\mu+1)}{2} z-(1-\mu) z(1-z) \partial_{z}\right) \mathbf{F}_{\alpha, \beta, \mu} \\
& =\frac{(1+\alpha+\beta-\mu)(-1-\alpha+\beta+\mu)}{4} \mathbf{F}_{\alpha, \beta, \mu-2}(z) .
\end{aligned}
$$

Note that (3.9) follows from the 6th and 7th recurrence relation, and (3.10) follows from the 5th and 8th of Sect. 3.9.

\subsection{Degenerate Case}

$\alpha=m \in \mathbb{Z}$ is the degenerate case of the hypergeometric equation at 0 . We have then

$$
\mathbf{F}(a, b ; 1+m ; z)=\sum_{n=\max (0,-m)} \frac{(a)_{n}(b)_{n}}{n !(m+n) !} z^{n} .
$$

This easily implies the identity

$$
(a-m)_{m}(b-m)_{m} \mathbf{F}(a, b ; 1+m ; z)=z^{-m} \mathbf{F}(a-m, b-m ; 1-m ; z) .
$$

Thus, the two standard solutions determined by the behavior at zero are proportional to one another.

One can also see the degenerate case in the integral representation (3.3). If we go around $0, z$, the phase of the integrand changes by $\mathrm{e}^{\mathrm{i} 2 \pi c}=\mathrm{e}^{\mathrm{i} 2 \pi \alpha}$. Therefore, if $\alpha=m \in \mathbb{Z}$, then the loop around $0, z$ is closed on the Riemann surface of the integrand. 
We have an additional integral representation and a generating function:

$$
\begin{aligned}
\frac{1}{2 \pi \mathrm{i}} \int_{\left[(0, z)^{+}\right]}(1-t)^{-a}(1-z / t)^{-b} t^{-m-1} \mathrm{~d} t & =(a)_{m} \mathbf{F}_{m, a+b-1,-a+b-m}(z) \\
& =z^{-m}(b)_{-m} \mathbf{F}_{-m, a+b-1, a-b+m}(z), \\
(1-t)^{-a}(1-z / t)^{-b} & =\sum_{m \in \mathbb{Z}} t^{m}(a)_{m} \mathbf{F}_{m, a+b-1, b-a-m}(z) .
\end{aligned}
$$

To see the integral representation, we note that the integral on the 1.h.s. is annihilated by the hypergeometric operator. Then, we check that its value at zero equals

$$
\frac{1}{2 \pi \mathrm{i}} \int_{\left[0^{+}\right]}(1-t)^{-a} t^{-m-1} \mathrm{~d} t=\frac{(a)_{m}}{m !}
$$

see $(2.24)$.

The second identity follows from (3.11). Another way to see it is to make the substitution $t=\frac{z}{s}$. Note that $\left[(0, z)^{+}\right]$becomes $\left[(\infty, 1)^{+}\right]$, which coincides with $\left[(0, z)^{-}\right]$. Then, we change the sign in front of the integral and the orientation of the contour of integration, obtaining

$$
\frac{z^{-m}}{2 \pi \mathrm{i}} \int_{\left[(0, z)^{+}\right]}(1-s)^{-b}(1-z / s)^{-a} s^{-m-1} \mathrm{~d} s .
$$

Finally, we apply the first integral representation again.

The generating function follows from the integral representation.

\subsection{Jacobi Polynomials}

If $-a=n=0,1, \ldots$, then hypergeometric functions are polynomials. We will call them the Jacobi polynomials.

Following Sect. 1.6, the Jacobi polynomials are defined by the Rodriguez-type formula

$$
R_{n}^{\alpha, \beta}(z):=\frac{(-1)^{n}}{n !} z^{-\alpha}(z-1)^{-\beta} \partial_{z}^{n} z^{\alpha+n}(z-1)^{\beta+n} .
$$

Remark 3.3. In most of the literature, the Jacobi polynomials are slightly different:

$$
P_{n}^{\alpha, \beta}(z):=R_{n}^{\alpha, \beta}\left(\frac{1-z}{2}\right)=(-1)^{n} R_{n}^{\beta, \alpha}\left(\frac{1+z}{2}\right) .
$$

The equation:

$$
\begin{aligned}
0 & =\mathcal{F}\left(-n, 1+\alpha+\beta+n ; \beta+1 ; z, \partial_{z}\right) P_{n}^{\alpha, \beta}(z) \\
& =\left(z(1-z) \partial_{z}^{2}+((1+\alpha)(1-z)-(1+\beta) z) \partial_{z}+n(n+\alpha+\beta+1)\right) P_{n}^{\alpha, \beta}(z) .
\end{aligned}
$$


Generating functions:

$$
\begin{aligned}
(1+t(1-z))^{\alpha}(1-t z)^{\beta} & =\sum_{n=0}^{\infty} t^{n} R_{n}^{\alpha-n, \beta-n}(z), \\
(1+z t)^{-1-\alpha-\beta}(1+t)^{\alpha} & =\sum_{n=0}^{\infty} t^{n} R_{n}^{\alpha-n, \beta}(z), \\
(1+(z-1) t)^{-1-\alpha-\beta}(1-t)^{\beta} & =\sum_{n=0}^{\infty} t^{n} R_{n}^{\alpha, \beta-n}(z) .
\end{aligned}
$$

Integral representations:

$$
\begin{aligned}
R_{n}^{\alpha, \beta}(z) & =\frac{1}{2 \pi \mathrm{i}} \int_{\left[0^{+}\right]}(1+(1-z) t)^{\alpha+n}(1-z t)^{\beta+n} t^{-n-1} \mathrm{~d} t \\
& =\frac{1}{2 \pi \mathrm{i}} \int_{\left[0^{+}\right]}(1+z t)^{-\alpha-\beta-n-1}(1+t)^{\alpha+n} t^{-n-1} \mathrm{~d} t \\
& =\frac{1}{2 \pi \mathrm{i}} \int_{\left[0^{+}\right]}(1+(z-1) t)^{-\alpha-\beta-n-1}(1-t)^{\beta+n} t^{-n-1} \mathrm{~d} t .
\end{aligned}
$$

Discrete symmetries:

$$
\begin{aligned}
R_{n}^{\alpha, \beta}(z) & =(1-z)^{n} R_{n}^{\alpha,-1-\alpha-\beta-2 n}\left(\frac{z}{z-1}\right) \\
& =(-1)^{n} R_{n}^{\beta, \alpha}(1-z)=(-z)^{n} R_{n}^{\beta,-1-\alpha-\beta-2 n}\left(\frac{z-1}{z}\right) \\
& =z^{n} R_{n}^{-1-\alpha-\beta-2 n, \beta}\left(\frac{1}{z}\right)=(z-1)^{n} R_{n}^{-1-\alpha-\beta-2 n, \alpha}\left(\frac{1}{1-z}\right) .
\end{aligned}
$$

Recurrence relations:

$$
\begin{gathered}
\partial_{z} R_{n}^{\alpha, \beta}(z)=-(\alpha+\beta+n+1) R_{n-1}^{\alpha+1, \beta+1}(z) \\
\left(z(1-z) \partial_{z}-\alpha(z-1)-\beta z\right) R_{n}^{\alpha, \beta}(z)=(n+1) R_{n+1}^{\alpha-1, \beta-1}(z) \\
\left((1-z) \partial_{z}-\beta\right) R_{n}^{\alpha, \beta}(z)=-(\beta+n) R_{n}^{\alpha-1, \beta+1}(z) \\
\left(z \partial_{z}+\alpha\right) R_{n}^{\alpha, \beta}(z)=(\beta+n) R_{n}^{\alpha-1, \beta+1}(z) \\
\left(z \partial_{z}-n\right) R_{n}^{\alpha, \beta}(z)=-(\alpha+n) R_{n-1}^{\alpha, \beta+1}(z) \\
\left(z(1-z) \partial_{z}+1+\alpha+n-(1+\alpha+\beta+n) z\right) R_{n}^{\alpha, \beta}(z)=(n+1) R_{n+1}^{\alpha, \beta-1}(z), \\
\left(z \partial_{z}+1+\alpha+\beta+n\right) R_{n}^{\alpha, \beta}(z)=(1+\alpha+\beta+n) R_{n}^{\alpha, \beta+1}(z), \\
\left(z(1-z) \partial_{z}-n-\beta+n z\right) R_{n}^{\alpha, \beta}(z)=-(\beta+n) R_{n}^{\alpha, \beta-1}(z), \\
\left((z-1) \partial_{z}-n\right) R_{n}^{\alpha, \beta}(z)=(\beta+n) R_{n-1}^{\alpha+1, \beta}(z), \\
\left(z(1-z) \partial_{z}+\alpha-(1+\alpha+\beta+n) z\right) R_{n}^{\alpha, \beta}(z)=(n+1) R_{n+1}^{\alpha-1, \beta}(z)
\end{gathered}
$$




$$
\begin{gathered}
\left((z-1) \partial_{z}+1+n+\alpha+\beta\right) R_{n}^{\alpha, \beta}(z)=(1+n+\alpha+\beta) R_{n}^{\alpha+1, \beta}(z), \\
\left(z(1-z) \partial_{z}+\alpha+n z\right) R_{n}^{\alpha, \beta}(z)=(n+\alpha) R_{n}^{\alpha-1, \beta}(z) .
\end{gathered}
$$

The first, second, resp. third integral representation is easily seen to be equivalent to the first, second, resp. third generating function. The first follows immediately from the Rodriguez-type formula.

The symmetries can be interpreted as a subset of Kummer's table. The first line corresponds to the symmetries of the solution regular at 0 , see (3.7) (or Sect. 3.7.1). Note that from 4 expressions in (3.7) only the first and the third survive, since $n=-a$ should not change. The second line corresponds to the solution regular at 1 (Sect. 3.7.3), finally the third line to the solution $\sim z^{-a}=z^{n}$ (Sect. 3.7.5).

The differential equation, the Rodriguez-type formula, the first generating function, the first integral representation and the first pair of recurrence relations are special cases of the corresponding formulas of Sect. 1.6.

Note that Jacobi polynomials are regular at 0,1 , and behave as $z^{n}$ in infinity. Thus (up to coefficients), they coincide with the 3 standard solutions. They have the following values at 0,1 and the behavior at $\infty$ :

$$
\begin{aligned}
R_{n}^{\alpha, \beta}(0) & =\frac{(\alpha+1)_{n}}{n !}, \quad R_{n}^{\alpha, \beta}(1)=(-1)^{n} \frac{(\beta+1)_{n}}{n !}, \\
\lim _{z \rightarrow \infty} \frac{R_{n}^{\alpha, \beta}(z)}{z^{n}} & =(-1)^{n} \frac{(\alpha+\beta+n+1)_{n}}{n !} .
\end{aligned}
$$

We have several alternative expressions for Jacobi polynomials:

$$
\begin{aligned}
R_{n}^{\alpha, \beta}(z) & :=\lim _{\nu \rightarrow n}(-1)^{n}(\nu-n) \mathbf{F}_{\alpha, \beta, 2 \nu+\alpha+\beta+1}^{\mathrm{I}}(z)=\frac{(\alpha+1)_{n}}{n !} F_{\alpha, \beta, 2 n+\alpha+\beta+1}(z) \\
& =\frac{\Gamma(\alpha+1+n)}{\Gamma(\alpha+1) \Gamma(n+1)} F(-n, n+\alpha+\beta+1 ; \alpha+1 ; z) \\
& =\sum_{j=0}^{n} \frac{(1+\alpha+j)_{n-j}(1+\alpha+\beta+n)_{j}}{j !(n-j) !}(-z)^{j} .
\end{aligned}
$$

One way to derive the first of the above identities is to use integral representation (3.6). Using that $a$ is an integer we can replace the open curve $\left[1,(0, z)^{+}, 1\right]$ with a closed loop $\left[\infty^{-}\right]$:

$$
\begin{aligned}
\lim _{\nu \rightarrow n}(-1)^{n}(\nu-n) \mathbf{F}_{\alpha, \beta, 2 \nu+\alpha+\beta+1}^{\mathrm{I}}(z) \\
\quad=\lim _{\nu \rightarrow n} \frac{\sin \nu \pi}{\pi} \mathbf{F}_{\alpha, \beta, 2 \nu+\alpha+\beta+1}^{\mathrm{I}}(z) \\
\quad=\frac{1}{2 \pi \mathrm{i}} \int_{\left[\infty^{-}\right]}(-s)^{\beta+n}(1-s)^{\alpha+n}(z-s)^{-1-\alpha-\beta-n} \mathrm{~d} s .
\end{aligned}
$$


Then, making the substitutions $s=z-\frac{1}{t}, s=z t$, resp. $s=(z-1) t$, we obtain the 1st, 2nd, resp. 3rd integral representation.

Additional identities valid in the degenerate case:

$$
\begin{aligned}
& R_{n}^{\alpha, \beta}(z)=\frac{(n+1)_{\alpha}}{(\beta+n+1)_{\alpha}}(-z)^{-\alpha} R_{n+\alpha}^{-\alpha, \beta}(z), \quad \alpha \in \mathbb{Z} ; \\
& R_{n}^{\alpha, \beta}(z)=\frac{(n+1)_{\beta}}{(\alpha+n+1)_{\beta}}(1-z)^{-\beta} R_{n+\beta}^{\alpha,-\beta}(z), \quad \beta \in \mathbb{Z} \\
& R_{n}^{\alpha, \beta}(z)=(-z)^{-\alpha}(1-z)^{-\beta} R_{n+\alpha+\beta}^{-\alpha,-\beta}(z), \quad \alpha, \beta \in \mathbb{Z} .
\end{aligned}
$$

There is a region where Jacobi polynomials are zero. This happens iff $\alpha, \beta \in \mathbb{Z}$ and $\alpha, \beta$ are in the triangle

$$
\begin{aligned}
& 0 \leq \alpha+n \\
& 0 \leq \beta+n \\
& 0 \leq-\alpha-\beta-n-1
\end{aligned}
$$

In the analysis of symmetries of Jacobi polynomials, it is useful to go back to the Lie-algebraic parameters, more precisely, to set $\mu:=-\alpha-\beta-2 n-1$. Then (3.12) acquires a more symmetric form, since we can replace its last condition by

$$
0 \leq \mu+n
$$

One can distinguish three strips where Jacobi polynomials have special properties. Note that the intersection of the strips below is precisely the triangle described in (3.12).

1. $\mu \in \mathbb{Z}$ and $-n \leq \mu \leq-1$ or, equivalently, $\alpha+\beta \in \mathbb{Z}$ and $-2 n \leq \alpha+\beta \leq$ $-n-1$. Then $R_{n}^{\alpha, \beta}=0$ or

$$
\operatorname{deg} R_{n}^{\alpha, \beta}=\mu+n=-\alpha-\beta-n-1
$$

2. $\alpha \in \mathbb{Z}$ and $-n \leq \alpha \leq-1$. Then $R_{n}^{\alpha, \beta}=0$ or

$$
R_{n}^{\alpha, \beta}=z^{-\alpha} W, \quad W \text { not divisible by } z \text {. }
$$

3. $\beta \in \mathbb{Z}$ and $-n \leq \beta \leq-1$. Then $R_{n}^{\alpha, \beta}=0$ or

$$
R_{n}^{\alpha, \beta}=(z-1)^{-\beta} V, \quad V \text { not divisible by } z-1 \text {. }
$$

These regions are presented in the following picture: 


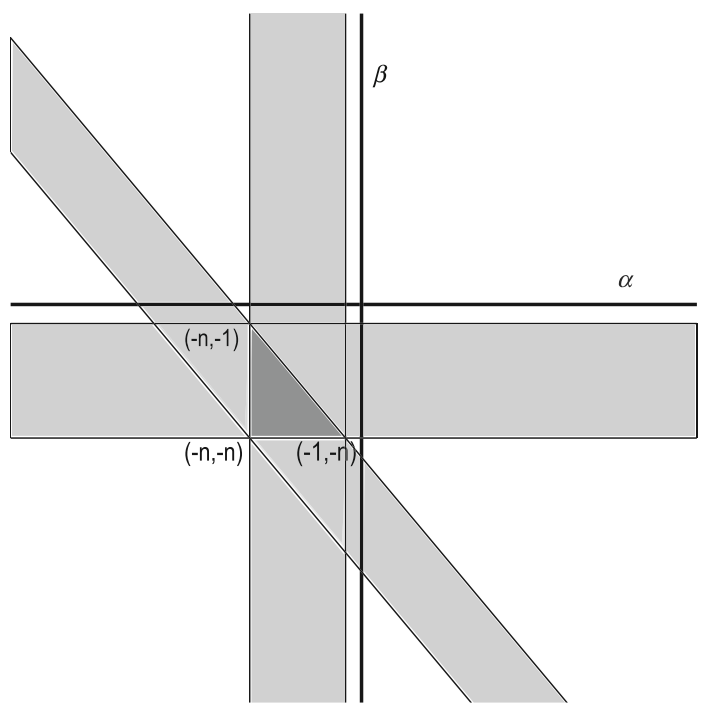

Finally, Jacobi polynomials satisfy some identities related to Sect. 3.10. An additional generating function:

$$
\begin{aligned}
& 2^{\alpha+\beta} r^{-1}(1-t+r)^{-\alpha}(1+t+r)^{-\beta}=\sum_{n=0}^{\infty} t^{n} R_{n}^{\alpha, \beta}(z), \\
& \text { where } r=\sqrt{(1-t)^{2}+4 z t} .
\end{aligned}
$$

Additional recurrence relations:

$$
\begin{aligned}
& ((n+\alpha+\beta+1)((n+\beta+1)-(2 n+\alpha+\beta+2) z) \\
& \left.+(2 n+\alpha+\beta+2) z(1-z) \partial_{z}\right) R_{n}^{\alpha, \beta}(z)=(n+\alpha+\beta+1)(n+1) R_{n+1}^{\alpha, \beta}(z), \\
& \quad\left(n((n+\alpha)-(2 n+\alpha+\beta) z)-(2 n+\alpha+\beta) z(1-z) \partial_{z}\right) R_{n}^{\alpha, \beta}(z) \\
& \quad=(n+\alpha)(n+\beta) R_{n-1}^{\alpha, \beta}(z) .
\end{aligned}
$$

\subsection{Special Cases}

Beside the polynomial and degenerate cases, the hypergeometric equation has a number of other special cases. In their description most of the time, we will use the Lie-algebraic parameters, which are here more convenient than the classical parameters.

3.13.1. Gegenbauer Equation Through an Affine Transformation. Consider a hypergeometric equation whose two parameters coincide up to a sign. After applying an appropriate symmetry, we can assume that they are at the first and second place, and that they are equal to one another. In other words, $\alpha=\beta$. A simple affine transformation (6.2) can be then applied to obtain a reflection invariant equation called the Gegenbauer equation. We study it separately in Sect. 6 . 
3.13.2. Gegenbauer Equation Through a Quadratic Transformation. Hypergeometric equations with one of the parameters equal to $\frac{1}{2}$ or $-\frac{1}{2}$ also enjoy special properties. After applying, if needed, one of the symmetries, we can assume that $\mu= \pm \frac{1}{2}$. Then identity (6.4) or (6.5) leads to the Gegenbauer equation.

3.13.3. Chebyshev Equation. Even more special properties have equations with a pair of parameters $\pm \frac{1}{2}$. After applying one of the symmetries, we can assume that $\alpha=\beta=\frac{1}{2}$. Thus, we are reduced to the Chebyshev equation of the first kind; see (6.15). Another option is to reduce it to the Chebyshev equation of the second kind, which corresponds to $\alpha=\beta=-\frac{1}{2}$; see (6.16).

3.13.4. Legendre Equation. Let $\mathcal{L}$ be the sublattice of $\mathbb{Z}^{3}$ consisting of points whose sum of coordinates is even. It is a sublattice of $\mathbb{Z}^{3}$ of degree 2 . Using recurrence relations of Sect. 3.9, we can pass from hypergeometric functions with given Lie-algebraic parameters $(\alpha, \beta, \mu)$ to parameters from $(\alpha, \beta, \mu)+\mathcal{L}$.

This is especially useful in the degenerate case, when some of the parameters are integers. In particular, if two of the parameters are integers, by applying recurrence relations we can make both of them zero. By applying an appropriate symmetry, we can assume that $\alpha=\beta=0$. Thus, we obtain the Legendre equation, see (6.14).

3.13.5. Elementary Solutions. One can easily check that

$$
F(a, b ; b ; ; z)=F_{b-1, a, b-a}(z)=(1-z)^{-a} .
$$

Therefore, using Kummer's table and recurrence relations, we see that if

$$
\epsilon_{1} \alpha+\epsilon_{2} \beta+\epsilon_{3} \mu \text { is an odd integer for some } \epsilon_{1}, \epsilon_{2}, \epsilon_{3} \in\{-1,1\}
$$

then $F_{\alpha, \beta, \mu}$ is an elementary function involving power functions, but not logarithms.

3.13.6. Fully Degenerate Case. An interesting situation arises if $\alpha, \beta, \mu \in \mathbb{Z}$, that is, we have the degenerate case at all singular points. We can distinguish two situations:

1. If $\alpha+\beta+\mu$ is even, by walking on the lattice $\mathcal{L}$ we can reduce ourselves to the equation for the complete elliptic integral, which corresponds to $\alpha=\beta=\mu=0$.

2. If $\alpha+\beta+\mu$ is odd, by walking on the lattice $\mathcal{L}$ we can reduce ourselves to the equation for the Legendre polynomial of degree 0 , which corresponds to $\alpha=\beta=0, \mu=1$. This equation is solved by

$$
\begin{gathered}
F_{0,0,1}(z)=F(0,1 ; 1 ; z)=1, \\
z^{-1} F_{1,0,0}\left(1-z^{-1}\right)=z^{-1} F\left(1,1 ; 2 ; 1-z^{-1}\right)=\log (z-1)-\log z,
\end{gathered}
$$

where we used Kummer's table and

$$
F(1,1 ; 2 ; w)=-w^{-1} \log (1-w) .
$$




\section{The ${ }_{1} F_{1}$ and ${ }_{2} F_{0}$ Equation}

\subsection{The ${ }_{1} F_{1}$ Equation}

Let $a, c \in \mathbb{C}$. The confluent or the ${ }_{1} F_{1}$ equation is given by the operator

$$
\mathcal{F}\left(a ; c ; z, \partial_{z}\right):=z \partial_{z}^{2}+(c-z) \partial_{z}-a .
$$

This equation is a limiting case of the hypergeometric equation:

$$
\lim _{b \rightarrow \infty} \frac{1}{b} \mathcal{F}\left(a, b ; c ; z / b, \partial_{z / b}\right)=\mathcal{F}\left(a ; c ; z, \partial_{z}\right) .
$$

\subsection{The ${ }_{2} F_{0}$ Equation}

Parallel to the ${ }_{1} F_{1}$ equation, we will consider the ${ }_{2} F_{0}$ equation, given by the operator

$$
\mathcal{F}\left(a, b ;-; z, \partial_{z}\right):=z^{2} \partial_{z}^{2}+(-1+(1+a+b) z) \partial_{z}+a b,
$$

where $a, b \in \mathbb{C}$. This equation is another limiting case of the hypergeometric equation:

$$
\lim _{c \rightarrow \infty} \mathcal{F}\left(a, b ; c ; c z, \partial_{(c z)}\right)=-\mathcal{F}\left(a, b ;-; z, \partial_{z}\right) .
$$

\subsection{Equivalence of the ${ }_{1} F_{1}$ and ${ }_{2} F_{0}$ Equation}

Note that

$$
\mathcal{F}\left(a, b ;-; z, \partial_{z}\right)=w^{2} \partial_{w}^{2}+\left(-w^{2}+(1-a-b) w\right) \partial_{w}+a b
$$

where $w=-z^{-1}, z=-w^{-1}$. Moreover,

$$
(-z)^{a+1} \mathcal{F}\left(a, b ;-; z, \partial_{z}\right)(-z)^{-a}=\mathcal{F}\left(a ; 1+a-b ; w, \partial_{w}\right) .
$$

Hence the ${ }_{2} F_{0}$ equation is equivalent to the ${ }_{1} F_{1}$ equation. We will treat the ${ }_{1} F_{1}$ equation as the principal one.

The relationship between the parameters is

$$
c=1+a-b, \quad b=1+a-c .
$$

\subsection{Lie-Algebraic Parameters}

Instead of the classical parameters, we usually prefer the Lie-algebraic parameters $\alpha, \theta$ :

$$
\begin{array}{ll}
\alpha:=c-1=a-b, & \theta:=-c+2 a=-1+a+b ; \\
a=\frac{1+\alpha+\theta}{2}, \quad b=\frac{1-\alpha+\theta}{2}, & c=1+\alpha .
\end{array}
$$

In these parameters, the ${ }_{1} F_{1}$ operator (4.1) becomes

$$
\mathcal{F}_{\theta, \alpha}\left(z, \partial_{z}\right)=z \partial_{z}^{2}+(1+\alpha-z) \partial_{z}-\frac{1}{2}(1+\theta+\alpha)
$$

and the ${ }_{2} F_{0}$ operator $(4.2)$ becomes

$$
\tilde{\mathcal{F}}_{\theta, \alpha}\left(z, \partial_{z}\right)=z^{2} \partial_{z}^{2}+(-1+(2+\theta) z) \partial_{z}+\frac{1}{4}(1+\theta)^{2}-\frac{1}{4} \alpha^{2} .
$$

The Lie-algebraic parameters have an interesting interpretation in terms of a natural basis of a "Cartan algebra" of the Lie algebra $\operatorname{sch}(2)$ [2]. 


\subsection{Integral Representations}

Two kinds of integral representations of solutions to the ${ }_{1} F_{1}$ equation are described below:

Theorem 4.1. 1. Let $[0,1] \ni t \mapsto \gamma(t)$ satisfy

$$
\left.t^{a-c+1} \mathrm{e}^{t}(t-z)^{-a-1}\right|_{\gamma(0)} ^{\gamma(1)}=0 .
$$

Then

$$
\mathcal{F}\left(a ; c ; z, \partial_{z}\right) \int_{\gamma} t^{a-c} \mathrm{e}^{t}(t-z)^{-a} \mathrm{~d} t=0 .
$$

2. Let $[0,1] \ni t \mapsto \gamma(t)$ satisfy

$$
\left.\mathrm{e}^{\frac{z}{t}} t^{-c}(1-t)^{c-a}\right|_{\gamma(0)} ^{\gamma(1)}=0 .
$$

Then

$$
\mathcal{F}\left(a ; c ; z, \partial_{z}\right) \int_{\gamma} \mathrm{e}^{\frac{z}{t}} t^{-c}(1-t)^{c-a-1} \mathrm{~d} t=0
$$

Proof. We check that for any contour $\gamma$ the l.h.s of (4.4) and (4.5) equal

$$
\begin{aligned}
& -a \int_{\gamma}\left(\partial_{t} t^{a-c+1} \mathrm{e}^{t}(t-z)^{-a-1}\right) \mathrm{d} t, \\
& -\int_{\gamma}\left(\partial_{t} \mathrm{e}^{\frac{z}{t}} t^{-c}(1-t)^{c-a}\right) \mathrm{d} t
\end{aligned}
$$

respectively.

For solutions of the ${ }_{2} F_{0}$ equation, we also have two kinds of integral representations:

Theorem 4.2. Let $[0,1] \ni t \mapsto \gamma(t)$ satisfy

$$
\left.\mathrm{e}^{-\frac{1}{t}} t^{b-a-1}(t-z)^{-b-1}\right|_{\gamma(0)} ^{\gamma(1)}=0
$$

Then

$$
\mathcal{F}\left(a, b ;-; z, \partial_{z}\right) \int_{\gamma} \mathrm{e}^{-\frac{1}{t}} t^{b-a-1}(t-z)^{-b} \mathrm{~d} t
$$

Proof. We check that for any contour $\gamma(4.6)$ equals

$$
-b \int_{\gamma}\left(\partial_{t} \mathrm{e}^{-\frac{1}{t}} t^{b-a-1}(t-z)^{-b-1}\right) \mathrm{d} t .
$$

The second integral representation is obtained if we interchange $a$ and $b$. 


\subsection{Symmetries}

The following operators equal $\mathcal{F}_{\theta, \alpha}\left(w, \partial_{w}\right)$ for the appropriate $w$ :

$$
\begin{array}{llll}
w=z: & & \\
& & \mathcal{F}_{\theta, \alpha}\left(z, \partial_{z}\right), & \\
& z^{-\alpha} & \mathcal{F}_{\theta,-\alpha}\left(z, \partial_{z}\right) & z^{\alpha}, \\
& & & \\
& -\mathrm{e}^{-z} & \mathcal{F}_{-\theta, \alpha}\left(z, \partial_{z}\right) & \mathrm{e}^{z}, \\
& -\mathrm{e}^{-z} z^{-\alpha} & \mathcal{F}_{-\theta,-\alpha}\left(z, \partial_{z}\right) & \mathrm{e}^{z} z^{\alpha} .
\end{array}
$$

The third symmetry is sometimes called the 1 st Kummer transformation.

Symmetries of the ${ }_{1} F_{1}$ operators can be interpreted as the "Weyl group" of the Lie algebra $\operatorname{sch}(2)$.

\subsection{Factorizations and Commutation Relations}

There are several ways of factorizing the ${ }_{1} F_{1}$ operator.

$$
\begin{aligned}
\mathcal{F}_{\theta, \alpha} & =\left(z \partial_{z}+1+\alpha-z\right) \partial_{z}-\frac{1}{2}(\theta+\alpha+1), \\
& =\partial_{z}\left(z \partial_{z}+\alpha-z\right)-\frac{1}{2}(\theta+\alpha-1), \\
& =\left(z \partial_{z}+1+\alpha\right)\left(\partial_{z}-1\right)+\frac{1}{2}(-\theta+\alpha+1), \\
& =\left(\partial_{z}-1\right)\left(z \partial_{z}+\alpha\right)+\frac{1}{2}(-\theta+\alpha-1) ; \\
z \mathcal{F}_{\theta, \alpha}= & \left(z \partial_{z}+\frac{1}{2}(\theta+\alpha-1)\right)\left(z \partial_{z}+\frac{1}{2}(-\theta+\alpha+1)-z\right) \\
& -\frac{1}{4}(-\theta+\alpha+1)(\theta+\alpha-1), \\
= & \left(z \partial_{z}+\frac{1}{2}(-\theta+\alpha-1)-z\right)\left(z \partial_{z}+\frac{1}{2}(\theta+\alpha+1)\right) \\
& -\frac{1}{4}(-\theta+\alpha-1)(\theta+\alpha+1) .
\end{aligned}
$$

One can use the factorizations to derive the following commutation relations:

$$
\begin{aligned}
\partial_{z} & \mathcal{F}_{\theta, \alpha} \\
=\mathcal{F}_{\theta+1, \alpha+1} & \partial_{z}, \\
\left(z \partial_{z}+\alpha-z\right) & \mathcal{F}_{\theta, \alpha} \\
=\mathcal{F}_{\theta-1, \alpha-1} & \left(z \partial_{z}+\alpha-z\right), \\
\left(z \partial_{z}+\alpha\right) & \mathcal{F}_{\theta, \alpha} \\
=\mathcal{F}_{\theta+1, \alpha-1} & \left(z \partial_{z}+\alpha\right), \\
\left(\partial_{z}-1\right) & \mathcal{F}_{\theta, \alpha} \\
=\mathcal{F}_{\theta-1, \alpha+1} & \left(\partial_{z}-1\right) \\
\left(z \partial_{z}+\frac{1}{2}(\theta+\alpha+1)\right) & z \mathcal{F}_{\theta, \alpha} \\
=z \mathcal{F}_{\theta+2, \alpha} & \left(z \partial_{z}+\frac{1}{2}(\theta+\alpha+1)\right) \\
\left(z \partial_{z}+\frac{1}{2}(-\theta+\alpha+1)-z\right) & z \mathcal{F}_{\theta, \alpha} \\
=z \mathcal{F}_{\theta-2, \alpha} & \left(z \partial_{z}+\frac{1}{2}(-\theta+\alpha+1)-z\right) .
\end{aligned}
$$


Each of these commutation relations can be associated with a "root" of the Lie algebra $\operatorname{sch}(2)$.

\subsection{Canonical Forms}

The natural weight of the ${ }_{1} F_{1}$ operator equals $z^{\alpha} \mathrm{e}^{-z}$, so that

$$
\mathcal{F}_{\theta, \alpha}=z^{-\alpha} \mathrm{e}^{z} \partial_{z} z^{\alpha+1} \mathrm{e}^{-z} \partial_{z}-\frac{1}{2}(1+\alpha+\theta)
$$

The balanced form of the ${ }_{1} F_{1}$ operator is

$$
z^{\frac{\alpha}{2}} \mathrm{e}^{-\frac{z}{2}} \mathcal{F}_{\theta, \alpha} z^{-\frac{\alpha}{2}} \mathrm{e}^{\frac{z}{2}}=\partial_{z} z \partial_{z}-\frac{z}{4}-\frac{\theta}{2}-\frac{\alpha^{2}}{4 z} .
$$

Remark 4.3. We have

$$
\begin{aligned}
2 z^{\frac{\alpha}{2}-1} \mathrm{e}^{-\frac{z}{2}} \mathcal{F}_{0, \alpha}\left(z, \partial_{z}\right) z^{-\frac{\alpha}{2}} \mathrm{e}^{\frac{z}{2}}=\partial_{w}^{2}+\frac{1}{w} \partial_{w}-1-\frac{\alpha^{2}}{w^{2}}, & z=2 w \\
2 \mathrm{i} z^{\frac{\alpha}{2}-1} \mathrm{e}^{-\frac{z}{2}} \mathcal{F}_{0, \alpha}\left(z, \partial_{z}\right) z^{-\frac{\alpha}{2}} \mathrm{e}^{\frac{z}{2}}=\partial_{u}^{2}+\frac{1}{u} \partial_{u}+1-\frac{\alpha^{2}}{u^{2}}, & z=2 \mathrm{i} u
\end{aligned}
$$

which are the operators for the modified Bessel and Bessel equations. Thus, both these equations essentially coincide with the balanced form of the ${ }_{1} F_{1}$ equation with $\theta=0$. We will discuss them further in Remark 5.3.

The Schrödinger form of the ${ }_{1} F_{1}$ equation is

$$
z^{\frac{\alpha}{2}-\frac{1}{2}} \mathrm{e}^{-\frac{z}{2}} \mathcal{F}_{\theta, \alpha} z^{-\frac{\alpha}{2}-\frac{1}{2}} \mathrm{e}^{\frac{z}{2}}=\partial_{z}^{2}-\frac{1}{4}-\frac{\theta}{2 z}+\left(\frac{1}{4}-\frac{\alpha^{2}}{4}\right) \frac{1}{z^{2}} .
$$

Remark 4.4. In the literature, the equation given by (4.7) is often called the Whittaker equation. Its standard form is

$$
\partial_{z}^{2}-\frac{1}{4}+\frac{\kappa}{z}+\left(\frac{1}{4}-\mu^{2}\right) \frac{1}{z^{2}}
$$

Thus, $\kappa, \mu$ correspond to $-\frac{\theta}{2}, \frac{\alpha}{2}$.

The natural weight of the ${ }_{2} F_{0}$ operator equals $z^{\theta} \mathrm{e}^{\frac{1}{z}}$, so that

$$
\tilde{\mathcal{F}}_{\theta, \alpha}=z^{-\theta} \mathrm{e}^{-\frac{1}{z}} \partial_{z} z^{\theta+2} \mathrm{e}^{\frac{1}{z}} \partial_{z}+\frac{(1+\theta)^{2}}{4}-\frac{\alpha^{2}}{4} .
$$

The balanced form of the ${ }_{2} F_{0}$ operator is

$$
z^{\frac{\theta}{2}} \mathrm{e}^{\frac{1}{2 z}} \tilde{\mathcal{F}}_{\theta, \alpha} z^{-\frac{\theta}{2}} \mathrm{e}^{-\frac{1}{2 z}}=\partial_{z} z^{2} \partial_{z}-\frac{1}{4 z^{2}}+\frac{\theta}{2 z}+\frac{1-\alpha^{2}}{4} .
$$

The symmetries $\alpha \mapsto-\alpha$, as well as $(z, \theta) \mapsto(-z,-\theta)$, are obvious in both balanced forms and in the Whittaker equation. 


\subsection{The ${ }_{1} F_{1}$ Function}

Equation (4.1) has a regular singular point at 0 . Its indices at 0 are equal to $0,1-c$. For $c \neq 0,-1,-2, \ldots$, the unique solution of the confluent equation analytic at 0 and equal to 1 at 0 is called the ${ }_{1} F_{1}$ hypergeometric function or the confluent function. It is equal to

$$
F(a ; c ; z):=\sum_{n=0}^{\infty} \frac{(a)_{n}}{(c)_{n}} \frac{z^{n}}{n !} .
$$

It is defined for $c \neq 0,-1,-2, \ldots$ Sometimes, it is more convenient to consider the function

$$
\mathbf{F}(a ; c ; z):=\frac{F(a ; c ; z)}{\Gamma(c)}=\sum_{n=0}^{\infty} \frac{(a)_{n}}{\Gamma(c+n)} \frac{z^{n}}{n !} .
$$

Another useful function proportional to ${ }_{1} F_{1}$ is

$$
\mathbf{F}^{\mathrm{I}}(a ; c ; z):=\frac{\Gamma(a) \Gamma(c-a)}{\Gamma(c)} F(a ; c ; z) .
$$

The confluent function can be obtained as the limit of the hypergeometric function:

$$
F(a ; c ; z)=\lim _{b \rightarrow \infty} F(a, b ; c ; z / b) .
$$

It satisfies the so-called Kummer's identity:

$$
F(a ; c ; z)=\mathrm{e}^{z} F(c-a ; c ;-z) .
$$

Integral representations for all parameters

$$
\frac{1}{2 \pi \mathrm{i}} \int_{]-\infty,(0, z)^{+},-\infty[} t^{a-c} \mathrm{e}^{t}(t-z)^{-a} \mathrm{~d} t=\mathbf{F}(a ; c ; z),
$$

for $\operatorname{Re} a>0, \operatorname{Re}(c-a)>0$

$$
\int_{[1,+\infty[} \mathrm{e}^{\frac{z}{t}} t^{-c}(t-1)^{c-a-1} \mathrm{~d} t=\mathbf{F}^{\mathrm{I}}(a ; c ; z),
$$

and for $\operatorname{Re}(c-a)>0$

$$
\frac{1}{2 \pi \mathrm{i}} \int_{\left[1,0^{+}, 1\right]} \mathrm{e}^{\frac{z}{t}}(-t)^{-c}(-t+1)^{c-a-1} \mathrm{~d} t=\frac{\sin \pi a}{\pi} \mathbf{F}^{\mathrm{I}}(a ; c ; z) .
$$

In the Lie-algebraic parameters:

$$
\begin{aligned}
F_{\theta, \alpha}(z) & :=F\left(\frac{1+\alpha+\theta}{2} ; 1+\alpha ; z\right), \\
\mathbf{F}_{\theta, \alpha}(z) & :=\mathbf{F}\left(\frac{1+\alpha+\theta}{2} ; 1+\alpha ; z\right) \\
& =\frac{1}{\Gamma(\alpha+1)} F_{\theta, \alpha}(z),
\end{aligned}
$$




$$
\begin{aligned}
\mathbf{F}_{\theta, \alpha}^{\mathrm{I}}(z) & :=\mathbf{F}^{\mathrm{I}}\left(\frac{1+\alpha+\theta}{2} ; 1+\alpha ; z\right) \\
& =\frac{\Gamma\left(\frac{1+\alpha+\theta}{2}\right) \Gamma\left(\frac{1+\alpha-\theta}{2}\right)}{\Gamma(\alpha+1)} F_{\theta, \alpha}(z) .
\end{aligned}
$$

Remark 4.5. In the literature, the ${ }_{1} F_{1}$ function is often called Kummer's function and denoted

$$
M(a, c, z):=F(a ; c ; z) .
$$

One also uses the Whittaker function of the 1st kind

$$
M_{\kappa, \mu}(z):=\exp (-z / 2) z^{\mu+1 / 2} M\left(\mu-\kappa+\frac{1}{2}, 1+2 \mu, z\right),
$$

which solves the Whittaker equation.

\subsection{The ${ }_{2} F_{0}$ Function}

We define, for $z \in \mathbb{C} \backslash[0,+\infty[$,

$$
F(a, b ;-; z):=\lim _{c \rightarrow \infty} F(a, b ; c ; c z),
$$

where $|\arg c-\pi|<\pi-\epsilon, \epsilon>0$. It extends to an analytic function on the universal cover of $\mathbb{C} \backslash\{0\}$ with a branch point of an infinite order at 0 . It has the following asymptotic expansion:

$$
F(a, b ;-; z) \sim \sum_{n=0}^{\infty} \frac{(a)_{n}(b)_{n}}{n !} z^{n}, \quad|\arg z-\pi|<\pi-\epsilon .
$$

Sometimes instead of ${ }_{2} F_{0}$, it is useful to consider the function

$$
\mathbf{F}^{\mathrm{I}}(a, b ;-; z):=\Gamma(a) F(a, b ;-; z) .
$$

We have an integral representation for $\operatorname{Re} a>0$

$$
\int_{0}^{\infty} \mathrm{e}^{-\frac{1}{t}} t^{b-a-1}(t-z)^{-b} \mathrm{~d} t=\mathbf{F}^{\mathrm{I}}(a, b ;-; z), \quad z \notin[0, \infty[,
$$

and without a restriction on parameters

$$
\frac{1}{2 \pi \mathrm{i}} \int_{\left[0, z^{+}, 0\right]} \mathrm{e}^{-\frac{1}{t}} t^{b-a-1}(t-z)^{-b} \mathrm{~d} t=\frac{\sin \pi a}{\pi} \mathbf{F}^{\mathrm{I}}(a, b ;-; z), \quad z \notin[0, \infty[.
$$

When we use the Lie-algebraic parameters, we denote the ${ }_{2} F_{0}$ function by $\tilde{F}$ and $\tilde{\mathbf{F}}$. The tilde is needed to avoid the confusion with the ${ }_{1} F_{1}$ function:

$$
\begin{aligned}
\tilde{F}_{\theta, \alpha}(z) & :=F\left(\frac{1+\alpha+\theta}{2}, \frac{1-\alpha+\theta}{2} ;-; z\right), \\
\tilde{\mathbf{F}}_{\theta, \alpha}^{\mathrm{I}}(z) & :=\mathbf{F}^{\mathrm{I}}\left(\frac{1+\alpha+\theta}{2}, \frac{1-\alpha+\theta}{2} ;-; z\right) \\
& =\Gamma\left(\frac{1-\alpha+\theta}{2}\right) \tilde{F}_{\theta, \alpha}(z) .
\end{aligned}
$$


Remark 4.6. In the literature, the ${ }_{2} F_{0}$ function is seldom used. Instead one uses Tricomi's function

$$
U(a, c, z):=z^{-a} F\left(a ; a-b-1 ;-; z^{-1}\right) .
$$

It is one of solutions of the ${ }_{1} F_{1}$ equation, which we will discuss in Sect. 4.11.3. One also uses the Whittaker function of the 2nd kind

$$
W_{\kappa, \mu}(z):=\exp (-z / 2) z^{\mu+1 / 2} U\left(\mu-\kappa+\frac{1}{2} ; 1+2 \mu ; z\right),
$$

which solves the Whittaker equation.

\subsection{Standard Solutions}

The ${ }_{1} F_{1}$ equation has two singular points. 0 is a regular singular point and with each of its two indices, we can associate the corresponding solution. $\infty$ is not a regular singular point. However, we can define two solutions with a simple behavior around $\infty$. Altogether we obtain 4 standard solutions, which we will describe in this subsection.

It follows by Theorem 4.1 that, for appropriate contours $\gamma_{1}, \gamma_{2}$, the integrals

$$
\begin{aligned}
& \int_{\gamma_{1}} t^{\frac{-1+\theta-\alpha}{2}} \mathrm{e}^{t}(t-z)^{\frac{-1-\theta-\alpha}{2}} \mathrm{~d} t, \\
& \int_{\gamma_{2}} \mathrm{e}^{\frac{z}{t}} t^{-1-\alpha}(t-1)^{\frac{-1-\theta+\alpha}{2}} \mathrm{~d} t
\end{aligned}
$$

solve the ${ }_{1} F_{1}$ equation.

In the first integral, the natural candidates for the endpoints of the intervals of integration are $\{-\infty, 0, z\}$. We will see that all 4 standard solutions can be obtained as such integrals.

In the second integral the natural candidates for endpoints are $\{1,0-$ $0, \infty\}$. (Recall from Sect. 2.3 that $0-0$ denotes 0 approached from the left). The 4 standard solutions can be obtained also from the integrals with these endpoints.

4.11.1. Solution $\sim \mathbf{1}$ at $\mathbf{0}$. For $\alpha \neq-1,-2, \ldots$, the only solution $\sim 1$ around 0 is

$$
F_{\theta, \alpha}(z)=\mathrm{e}^{z} F_{-\theta, \alpha}(-z) .
$$

The first integral representation is valid for all parameters:

$$
\frac{1}{2 \pi \mathrm{i}} \int_{]-\infty,(0, z)^{+-\infty}} t^{\frac{-1+\theta-\alpha}{2}} \mathrm{e}^{t}(t-z)^{\frac{-1-\theta-\alpha}{2}} \mathrm{~d} t=\mathbf{F}_{\theta, \alpha}(z) .
$$

The second is valid for $\operatorname{Re}(1+\alpha)>|\operatorname{Re} \theta|$ :

$$
\int_{[1,+\infty[} \mathrm{e}^{\frac{z}{t}} t^{-1-\alpha}(t-1)^{\frac{-1-\theta+\alpha}{2}} \mathrm{~d} t=\mathbf{F}_{\theta, \alpha}^{\mathrm{I}}(z) .
$$


4.11.2. Solution $\sim \boldsymbol{z}^{-\boldsymbol{\alpha}}$ at $\mathbf{0}$. If $\alpha \neq 1,2, \ldots$, then the only solution of the confluent equation behaving as $z^{-\alpha}$ at 0 is equal to

$$
z^{-\alpha} F_{\theta,-\alpha}(z)=z^{-\alpha} \mathrm{e}^{z} F_{-\theta,-\alpha}(-z) .
$$

Integral representation for $\operatorname{Re}(1-\alpha)>|\operatorname{Re} \theta|$ :

$$
\begin{gathered}
\left.\left.\int_{0}^{z} t^{\frac{-1+\theta-\alpha}{2}} \mathrm{e}^{t}(z-t)^{\frac{-1-\theta-\alpha}{2}} \mathrm{~d} t=z^{-\alpha} \mathbf{F}_{\theta,-\alpha}^{\mathrm{I}}(z), \quad z \notin\right]-\infty, 0\right] \\
\int_{z}^{0}(-t)^{\frac{-1+\theta-\alpha}{2}} \mathrm{e}^{t}(t-z)^{\frac{-1-\theta-\alpha}{2}} \mathrm{~d} t=(-z)^{-\alpha} \mathbf{F}_{\theta,-\alpha}^{\mathrm{I}}(z), \quad z \notin[0, \infty[;
\end{gathered}
$$

and without a restriction on parameters:

$$
\frac{1}{2 \pi \mathrm{i}} \int_{(0-0)^{+}} \mathrm{e}^{\frac{z}{t}} t^{-1-\alpha}(1-t)^{\frac{-1-\theta+\alpha}{2}} \mathrm{~d} t=z^{-\alpha} \mathbf{F}_{\theta,-\alpha}(z), \quad \operatorname{Re} z>0 .
$$

4.11.3. Solution $\sim z^{-a}$ at $+\infty$. The following solution to the confluent equation behaves as $\sim z^{-a}=z^{-\frac{1+\theta+\alpha}{2}}$ at $+\infty$ for $|\arg z|<\pi-\epsilon$ :

$$
z^{\frac{-1-\theta-\alpha}{2}} \tilde{F}_{\theta, \pm \alpha}\left(-z^{-1}\right) \text {. }
$$

Integral representations for $\operatorname{Re}(1+\theta-\alpha)>0$ :

$$
\left.\left.\int_{-\infty}^{0}(-t)^{\frac{-1+\theta-\alpha}{2}} \mathrm{e}^{t}(z-t)^{\frac{-1-\theta-\alpha}{2}} \mathrm{~d} t=z^{\frac{-1-\theta-\alpha}{2}} \tilde{\mathbf{F}}_{\theta, \alpha}^{\mathrm{I}}\left(-z^{-1}\right), \quad z \notin\right]-\infty, 0\right]
$$

and, for $\operatorname{Re}(1+\theta+\alpha)>0$ :

$$
\int_{-\infty}^{0} \mathrm{e}^{\frac{z}{t}}(-t)^{-1-\alpha}(1-t)^{\frac{-1+\theta+\alpha}{2}} \mathrm{~d} t=z^{\frac{-1-\theta-\alpha}{2}} \tilde{\mathbf{F}}_{\theta,-\alpha}^{\mathrm{I}}\left(-z^{-1}\right), \quad \operatorname{Re} z>0 .
$$

4.11.4. Solution $\sim(-z)^{-b} \mathrm{e}^{z}$ at $-\infty$. The following solution to the confluent equation behaves as $\sim(-z)^{-b} \mathrm{e}^{z}=(-z)^{\frac{1+\theta-\alpha}{2}} \mathrm{e}^{z}$ at $\infty$ for $|\arg z-\pi|<\pi-\epsilon$ :

$$
\mathrm{e}^{z}(-z)^{\frac{-1-\theta-\alpha}{2}} \tilde{F}_{-\theta, \pm \alpha}\left(z^{-1}\right) \text {. }
$$

Integral representation for $\operatorname{Re}(1+\theta+\alpha)>0$ :

$$
\int_{-\infty}^{z}(-t)^{\frac{-1+\theta-\alpha}{2}} \mathrm{e}^{t}(z-t)^{\frac{1-\theta-\alpha}{2}} \mathrm{~d} t=\mathrm{e}^{z}(-z)^{\frac{-1-\theta-\alpha}{2}} \tilde{\mathbf{F}}_{-\theta,-\alpha}^{\mathrm{I}}\left(z^{-1}\right), \quad z \notin[0, \infty[
$$

and for $\operatorname{Re}(1+\theta-\alpha)>0$ :

$$
\int_{0}^{1} \mathrm{e}^{\frac{z}{t}} t^{-1-\alpha}(1-t)^{\frac{-1+\theta+\alpha}{2}} \mathrm{~d} t=\mathrm{e}^{z}(-z)^{\frac{-1-\theta-\alpha}{2}} \tilde{\mathbf{F}}_{-\theta, \alpha}^{\mathrm{I}}\left(z^{-1}\right), \quad \operatorname{Re} z<0 .
$$




\subsection{Connection Formulas}

We decompose standard solutions in pair of solutions with a simple behavior around zero.

$$
\begin{aligned}
z^{\frac{-1-\theta-\alpha}{2}} \tilde{F}_{\theta, \pm \alpha}\left(-z^{-1}\right)= & \frac{\pi}{\sin \pi(-\alpha) \Gamma\left(\frac{1+\theta-\alpha}{2}\right)} \mathbf{F}_{\theta, \alpha}(z) \\
& +\frac{\pi}{\sin \pi \alpha \Gamma\left(\frac{1+\theta+\alpha}{2}\right)} z^{-\alpha} \mathbf{F}_{\theta,-\alpha}(z), \\
\mathrm{e}^{z}(-z)^{\frac{-1-\theta-\alpha}{2}} \tilde{F}_{-\theta, \pm \alpha}\left(z^{-1}\right)= & \frac{\pi}{\sin \pi(-\alpha) \Gamma\left(\frac{1-\theta-\alpha}{2}\right)} \mathbf{F}_{\theta, \alpha}(z) \\
& +\frac{\pi}{\sin \pi \alpha \Gamma\left(\frac{1-\theta+\alpha}{2}\right)}(-z)^{-\alpha} \mathbf{F}_{\theta,-\alpha}(z) .
\end{aligned}
$$

\subsection{Recurrence Relations}

The following recurrence relations follow easily from the commutation relations of Sect. 4.7:

$$
\begin{aligned}
\partial_{z} \mathbf{F}_{\theta, \alpha}(z) & =\frac{1+\theta+\alpha}{2} \mathbf{F}_{\theta+1, \alpha+1}(z), \\
\left(z \partial_{z}+\alpha-z\right) \mathbf{F}_{\theta, \alpha}(z) & =\mathbf{F}_{\theta-1, \alpha-1}(z) \\
\left(z \partial_{z}+\alpha\right) \mathbf{F}_{\theta, \alpha}(z) & =\mathbf{F}_{\theta+1, \alpha-1}(z), \\
\left(\partial_{z}-1\right) \mathbf{F}_{\theta, \alpha}(z) & =\frac{-1+\theta-\alpha}{2} \mathbf{F}_{\theta-1, \alpha+1}(z), \\
\left(z \partial_{z}+\frac{1+\theta+\alpha}{2}\right) \mathbf{F}_{\theta, \alpha}(z) & =\frac{1+\theta+\alpha}{2} \mathbf{F}_{\theta+2, \alpha}(z), \\
\left(z \partial_{z}+\frac{1-\theta+\alpha}{2}-z\right) \mathbf{F}_{\theta, \alpha}(z) & =\frac{1-\theta+\alpha}{2} \mathbf{F}_{\theta-2, \alpha}(z) .
\end{aligned}
$$

The recurrence relations for the ${ }_{2} F_{0}$ functions are similar:

$$
\begin{aligned}
\left(z \partial_{z}+\frac{1+\theta+\alpha}{2}\right) \tilde{\mathbf{F}}_{\theta, \alpha}^{\mathrm{I}}(z) & =\frac{1+\theta+\alpha}{2} \tilde{\mathbf{F}}_{\theta+1, \alpha+1}^{\mathrm{I}}(z), \\
\left(z^{2} \partial_{z}-1+\frac{1+\theta-\alpha}{2} z\right) \tilde{\mathbf{F}}_{\theta, \alpha}^{\mathrm{I}}(z) & =-\tilde{\mathbf{F}}_{\theta-1, \alpha-1}^{\mathrm{I}}(z), \\
\left(z \partial_{z}+\frac{1+\theta-\alpha}{2}\right) \tilde{\mathbf{F}}_{\theta, \alpha}^{\mathrm{I}}(z) & =\tilde{\mathbf{F}}_{\theta+1, \alpha-1}^{\mathrm{I}}(z), \\
\left(z^{2} \partial_{z}-1+\frac{1+\theta+\alpha}{2} z\right) \tilde{\mathbf{F}}_{\theta, \alpha}^{\mathrm{I}}(z) & =\frac{1-\theta+\alpha}{2} \tilde{\mathbf{F}}_{\theta-1, \alpha+1}^{\mathrm{I}}(z), \\
\partial_{z} \tilde{\mathbf{F}}_{\theta, \alpha}^{\mathrm{I}}(z) & =\frac{1+\theta+\alpha}{2} \tilde{\mathbf{F}}_{\theta+2, \alpha}^{\mathrm{I}}(z), \\
\left(z^{2} \partial_{z}-1-\theta z\right) \tilde{\mathbf{F}}_{\theta, \alpha}^{\mathrm{I}}(z) & =\frac{1-\theta+\alpha}{2} \tilde{\mathbf{F}}_{\theta-2, \alpha}^{\mathrm{I}}(z) .
\end{aligned}
$$




\subsection{Additional Recurrence Relations}

There exists an additional pair of recurrence relations:

$$
\begin{aligned}
& \left((1-\alpha) z^{2} \partial_{z}+\frac{(1-\alpha)(1-\alpha+\theta)}{2} z+\frac{-1-\theta+\alpha}{2}\right) \tilde{F}_{\theta, \alpha}(z)=\frac{-1-\theta+\alpha}{2} \tilde{F}_{\theta, \alpha-2}(z), \\
& \left((1+\alpha) z^{2} \partial_{z}+\frac{(1+\alpha)(1+\alpha+\theta)}{2} z+\frac{-1-\theta-\alpha}{2}\right) \tilde{F}_{\theta, \alpha}(z)=\frac{-1-\theta-\alpha}{2} \tilde{F}_{\theta, \alpha+2}(z) .
\end{aligned}
$$

\subsection{Degenerate Case}

$\alpha=m \in \mathbb{Z}$ is the degenerate case of the confluent equation at 0 . We have then

$$
\mathbf{F}(a ; 1+m ; z)=\sum_{n=\max (0,-m)} \frac{(a)_{n}}{n !(m+n) !} z^{n} .
$$

This easily implies the identity

$$
(a-m)_{m} \mathbf{F}(a ; 1+m ; z)=z^{-m} \mathbf{F}(a-m ; 1-m ; z) .
$$

Thus, the two standard solutions determined by the behavior at zero are proportional to one another.

One can also see the degenerate case in the integral representations:

$$
\begin{aligned}
\frac{1}{2 \pi \mathrm{i}} \int_{\left[(z, 0)^{+}\right]} \mathrm{e}^{t}(1-z / t)^{-a} t^{-m-1} \mathrm{~d} t & =\mathbf{F}_{-1+2 a-m, m}(z) \\
& =(a)_{-m} z^{-m} \mathbf{F}_{-1+2 a-m,-m}(z) \\
\frac{1}{2 \pi \mathrm{i}} \int_{\left[(0,1)^{+}\right]} \mathrm{e}^{z / t}(1-t)^{-a} t^{-m-1} \mathrm{~d} t & =(a)_{m} \mathbf{F}_{-1+2 a+m, m}(z) \\
& =z^{-m} \mathbf{F}_{-1+2 a+m,-m}(z) .
\end{aligned}
$$

The corresponding generating functions are

$$
\begin{aligned}
& \mathrm{e}^{t}(1-z / t)^{-a}=\sum_{m \in \mathbb{Z}} t^{m} \mathbf{F}_{-1+2 a-m, m}(z), \\
& \mathrm{e}^{z / t}(1-t)^{-a}=\sum_{m \in \mathbb{Z}} t^{m}(a)_{m} \mathbf{F}_{-1+2 a+m, m}(z) .
\end{aligned}
$$

\subsection{Laguerre Polynomials}

${ }_{1} F_{1}$ functions for $-a=n=0,1,2, \ldots$ are polynomials. They are known as Laguerre polynomials.

Following Sect. 1.6, they can be defined by the following version of the Rodriguez-type formula:

$$
L_{n}^{\alpha}(z):=\frac{1}{n !} \mathrm{e}^{z} z^{-\alpha} \partial_{z}^{n} \mathrm{e}^{-z} z^{n+\alpha} .
$$


The differential equation:

$$
\mathcal{F}\left(-n ; \alpha+1 ; z, \partial_{z}\right) L_{n}^{\alpha}(z)=\left(z \partial_{z}^{2}+(1+\alpha-z) \partial_{z}+n\right) L_{n}^{\alpha}(z)=0 .
$$

Generating functions:

$$
\begin{aligned}
\mathrm{e}^{-t z}(1+t)^{\alpha} & =\sum_{n=0}^{\infty} t^{n} L_{n}^{\alpha-n}(z), \\
(1-t)^{-\alpha-1} \exp \frac{t z}{t-1} & =\sum_{n=0}^{\infty} t^{n} L_{n}^{\alpha}(z) .
\end{aligned}
$$

Integral representations:

$$
\begin{aligned}
L_{n}^{\alpha}(z) & =\frac{1}{2 \pi \mathrm{i}} \int_{\left[0^{+}\right]} \mathrm{e}^{-t z}(1+t)^{\alpha+n} t^{-n-1} \mathrm{~d} t \\
& =\frac{1}{2 \pi \mathrm{i}} \int_{\left[0^{+}\right]}(1-t)^{-\alpha-1} \exp \left(\frac{t z}{t-1}\right) t^{-n-1} \mathrm{~d} t .
\end{aligned}
$$

Expression in terms of the Bessel polynomials (to be defined in the next subsection):

$$
L_{n}^{\alpha}(z)=z^{n} B_{n}^{-2 n-\alpha-1}\left(-z^{-1}\right)
$$

Recurrence relations:

$$
\begin{aligned}
\partial_{z} L_{n}^{\alpha}(z) & =-L_{n-1}^{\alpha+1}(z), \\
\left(z \partial_{z}+\alpha-z\right) L_{n}^{\alpha}(z) & =(n+1) L_{n+1}^{\alpha-1}(z), \\
\left(z \partial_{z}+\alpha\right) L_{n}^{\alpha}(z) & =(\alpha+n) L_{n}^{\alpha-1}(z), \\
\left(\partial_{z}-1\right) L_{n}^{\alpha}(z) & =-L_{n}^{\alpha+1}(z), \\
\left(z \partial_{z}-n\right) L_{n}^{\alpha}(z) & =-(n+\alpha) L_{n-1}^{\alpha}(z), \\
\left(z \partial_{z}+n+\alpha+1-z\right) L_{n}^{\alpha}(z) & =(n+1) L_{n+1}^{\alpha}(z) .
\end{aligned}
$$

The first, resp. second integral representation is easily seen to be equivalent to the first, resp. second generating function.

The differential equation, the Rodriguez-type formula, the first generating function, the first integral representation and the first pair of recurrence relations are special cases of the corresponding formulas of Sect. 1.6.

We have several alternative expressions for Laguerre polynomials:

$$
\begin{aligned}
L_{n}^{\alpha}(z) & =\lim _{\nu \rightarrow n}(-1)^{n}(\nu-n) \mathbf{F}_{1+\alpha-2 \nu, \alpha}^{\mathrm{I}}(z)=\frac{(1+\alpha)_{n}}{n !} F(-n ; 1+\alpha ; z) \\
& =z^{n} \lim _{\nu \rightarrow n}(\nu-n) \tilde{\mathbf{F}}_{1+\alpha-2 \nu, \alpha}^{\mathrm{I}}(z)=\frac{1}{n !}(-z)^{n} F\left(-n,-\alpha-n ;-;-z^{-1}\right) \\
& =\sum_{j=0}^{n} \frac{(1+\alpha+j)_{n-j}}{j !(n-j) !}(-z)^{j} .
\end{aligned}
$$


Let us derive the above identity using the integral representation (4.10). Using that $a$ is an integer, we can replace the open curve $\left[1,0^{+}, 1\right]$ with a closed loop $\left[\infty^{-}\right]$:

$$
\begin{aligned}
\lim _{\nu \rightarrow n}(-1)^{n}(\nu-n) \mathbf{F}_{1+\alpha-2 \nu, \alpha}^{\mathrm{I}}(z) \\
\quad=\lim _{\nu \rightarrow n} \frac{\sin \nu \pi}{\pi} \mathbf{F}_{1+\alpha-2 \nu, \alpha}^{\mathrm{I}}(z) \\
\quad=\frac{1}{2 \pi \mathrm{i}} \int_{\left[\infty^{-}\right]} \mathrm{e}^{\frac{z}{s}}(-s)^{-1-\alpha}(1-s)^{\alpha+n} \mathrm{~d} s .
\end{aligned}
$$

Then, we set $s=-\frac{1}{t}$, resp. $s=1-\frac{1}{t}$ to obtain the integral representations.

The value at 0 and behavior at $\infty$ :

$$
L_{n}^{\alpha}(0)=\frac{(\alpha+1)_{n}}{n !}, \quad \lim _{z \rightarrow \infty} \frac{L_{n}^{\alpha}(z)}{z^{n}}=\frac{(-1)^{n}}{n !} .
$$

An additional identity valid in the degenerate case:

$$
L_{n}^{\alpha}(z)=(n+1)_{\alpha}(-z)^{-\alpha} L_{n+\alpha}^{-\alpha}(z), \quad \alpha \in \mathbb{Z} .
$$

\subsection{Bessel Polynomials}

The ${ }_{2} F_{0}$ functions for $-a=n=0,1,2, \ldots$ are polynomials. Appropriately normalized they are called Bessel polynomials. They are seldom used in the literature, because they do not form an orthonormal basis in any weighted space and they are easily expressed in terms of Laguerre polynomials.

Following Sect. 1.6, they can be defined by the following version of the Rodriguez-type formula:

$$
B_{n}^{\theta}(z):=\frac{1}{n !} z^{-\theta} \mathrm{e}^{z^{-1}} \partial_{z}^{n} \mathrm{e}^{-z^{-1}} z^{\theta+2 n} .
$$

Differential equation:

$$
\begin{aligned}
\mathcal{F} & \left(-n, n+\theta+1 ;-; \partial_{z}, z\right) B_{n}^{\theta}(z) \\
& =\left(z^{2} \partial_{z}^{2}+(-1+(2+\theta) z) \partial_{z}-\frac{1}{2} n(1+\theta-\alpha)\right) B_{n}^{\theta}(z)=0 .
\end{aligned}
$$

Generating functions:

$$
\begin{aligned}
& \mathrm{e}^{-t}(1-t z)^{-\theta-1}=\sum_{n=0}^{\infty} t^{n} B_{n}^{\theta-n}(z), \\
& (1+t z)^{\theta} \exp \left(\frac{-t}{1+t z}\right)=\sum_{n=0}^{\infty} t^{n} B_{n}^{\theta-2 n}(z) .
\end{aligned}
$$

Integral representations:

$$
\begin{aligned}
B_{n}^{\theta}(z) & =\frac{1}{2 \pi \mathrm{i}} \int_{\left[0^{+}\right]} \mathrm{e}^{t}(1-t z)^{-\theta-n-1} t^{-n-1} \mathrm{~d} t \\
& =\frac{1}{2 \pi \mathrm{i}} \int_{\left[0^{+}\right]}(1+t z)^{\theta+2 n} \exp \left(\frac{-t}{1+t z}\right) t^{-n-1} \mathrm{~d} t .
\end{aligned}
$$


Expression in terms of the Laguerre polynomials:

$$
B_{n}^{\theta}(z)=(-z)^{n} L_{n}^{-\theta-2 n-1}\left(-z^{-1}\right) .
$$

Recurrence relations:

$$
\begin{aligned}
\left(z \partial_{z}+n+\theta+1\right) B_{n}^{\theta}(z) & =(n+\theta+1) B_{n}^{\theta+1}(z), \\
\left(z^{2} \partial_{z}-1-n z\right) B_{n}^{\theta}(z) & =-B_{n}^{\theta-1}(z), \\
\left(z \partial_{z}-n\right) B_{n}^{\theta}(z) & =-B_{n-1}^{\theta+1}(z), \\
\left(z^{2} \partial_{z}-1+(n+\theta+1) z\right) B_{n}^{\theta}(z) & =-(n+1) B_{n+1}^{\theta-1}(z), \\
\partial_{z} B_{n}^{\theta}(z) & =-(n+\theta+1) B_{n-1}^{\theta+2}(z), \\
\left(z^{2} \partial_{z}-1-\theta z\right) B_{n}^{\theta}(z) & =-(n+1) B_{n+1}^{\theta-2}(z) .
\end{aligned}
$$

Most of the above identities can be directly obtained from the corresponding identities about Laguerre polynomials.

The differential equation, the Rodriguez-type formula, the second generating function, the second integral representation and the last pair of recurrence relations are special cases of the corresponding formulas of Sect. 1.6.

We have several alternative expressions for Bessel polynomials:

$$
\begin{aligned}
B_{n}^{\theta}(z) & =\lim _{\nu \rightarrow n}(-1)^{n}(\nu-n) \tilde{\mathbf{F}}_{\theta,-1-\theta-2 n}^{\mathrm{I}}(z)=\frac{1}{n !} F(-n, n+\theta+1 ;-; z) \\
& =z^{n} \lim _{\nu \rightarrow n}(\nu-n) \mathbf{F}_{\theta,-1-\theta-2 \nu}^{\mathrm{I}}\left(-z^{-1}\right) \\
& =\frac{(1+\theta+n)_{n}}{n !}(-z)^{n} F\left(-n ;-\theta-2 n ;-z^{-1}\right) .
\end{aligned}
$$

The value at zero and behavior at $\infty$ :

$$
B_{n}^{\theta}(0)=\frac{1}{n !}, \quad \lim _{z \rightarrow \infty} \frac{B_{n}^{\theta}(z)}{z^{n}}=\frac{(-1)^{n}(n+\theta+1)_{n}}{n !} .
$$

Both for Laguerre and Bessel polynomials, there exist additional recurrence relations and a generating function. Below, we give a pair of such recurrence relations for Bessel polynomials.

$$
\begin{gathered}
\left((2+2 n+\theta) z^{2} \partial_{z}+(2+2 n+\theta)(n+\theta+1) z\right. \\
-(n+\theta+1)) B_{n}^{\theta}(z)=-(n+1)(n+\theta+1) B_{n+1}^{\theta}(z), \\
\left(-(2 n+\theta) z^{2} \partial_{z}+(2 n+\theta) n z+n\right) B_{n}^{\theta}(z)=B_{n-1}^{\theta}(z) .
\end{gathered}
$$

They correspond to an additional generating function

$$
\begin{aligned}
2^{\theta} r^{-1}(1+r)^{-\theta} \exp \left(\frac{2 t}{1+r}\right) & =\sum_{\substack{n=0 \\
\text { where }}}^{\infty} t^{n} B_{n}^{\theta}(z),=\sqrt{1+4 z t}
\end{aligned}
$$

\subsection{Special Cases}

Apart from the polynomial case and the degenerate case, the confluent equation has some other cases with special properties. 
4.18.1. Bessel Equation. If $\theta=0$, the confluent equation is equivalent to the (modified) Bessel equation, which we already remarked in Remark 4.3. By a square root substitution, it is also equivalent to the ${ }_{0} F_{1}$ equation; see (5.2).

4.18.2. Hermite Equation. If $\alpha= \pm \frac{1}{2}$, the confluent equation is equivalent to the Hermite equation by the quadratic substitutions (7.3) and (7.2).

\section{The ${ }_{0} F_{1}$ Equation}

\subsection{Introduction}

Let $c \in \mathbb{C}$. In this section, we will consider the ${ }_{0} F_{1}$ equation given by the operator

$$
\mathcal{F}\left(c ; z, \partial_{z}\right):=z \partial_{z}^{2}+c \partial_{z}-1 .
$$

It is a limiting case of the ${ }_{1} F_{1}$ and ${ }_{2} F_{1}$ operator:

$$
\lim _{a, b \rightarrow \infty} \frac{1}{a b} \mathcal{F}\left(a, b ; c ; z / a b, \partial_{(z / a b)}\right)=\lim _{a \rightarrow \infty} \frac{1}{a} \mathcal{F}\left(a ; c ; z / a, \partial_{(z / a)}\right)=\mathcal{F}\left(c ; z, \partial_{z}\right) .
$$

Instead of $c$, it is often more natural to use its Lie-algebraic parameter

$$
\alpha:=c-1, \quad c=\alpha+1 .
$$

Thus, we obtain the operator

$$
\mathcal{F}_{\alpha}\left(z, \partial_{z}\right):=z \partial_{z}^{2}+(\alpha+1) \partial_{z}-1
$$

The Lie-algebraic parameter has well-known interpretation in terms of the "Cartan element" of the Lie algebra aso(2), [2,15,16].

\subsection{Equivalence with a Subclass of the Confluent Equation}

The ${ }_{0} F_{1}$ equation can be reduced to a special class of the confluent equation by the so-called Kummer's 2nd transformation:

$$
\mathcal{F}\left(c ; z, \partial_{z}\right)=\frac{4}{w} \mathrm{e}^{-w / 2} \mathcal{F}\left(c-\frac{1}{2} ; 2 c-1 ; w, \partial_{w}\right) \mathrm{e}^{w / 2},
$$

where $w= \pm 4 \sqrt{z}, z=\frac{1}{16} w^{2}$. Using the Lie-algebraic parameters, this can be rewritten as

$$
\mathcal{F}_{\alpha}\left(z, \partial_{z}\right)=\frac{4}{w} \mathrm{e}^{-w / 2} \mathcal{F}_{0,2 \alpha}\left(w, \partial_{w}\right) \mathrm{e}^{w / 2}
$$

\subsection{Integral Representations}

There are two kinds of integral representations of solutions to the ${ }_{0} F_{1}$ equation. Theorem 5.1 describes representations of the first kind, which will be called Bessel-Schläfli representations. They will be treated as the main ones.

Theorem 5.1. Suppose that $[0,1] \ni t \mapsto \gamma(t)$ satisfies

$$
\left.\mathrm{e}^{t} \mathrm{e}^{\frac{z}{t}} t^{-c}\right|_{\gamma(0)} ^{\gamma(1)}=0
$$


Then

$$
\mathcal{F}\left(c ; z, \partial_{z}\right) \int_{\gamma} \mathrm{e}^{t} \mathrm{e}^{\frac{z}{t}} t^{-c} \mathrm{~d} t=0 .
$$

Proof. We check that for any contour $\gamma(5.4)$ equals

$$
-\int_{\gamma}\left(\partial_{t} \mathrm{e}^{t} \mathrm{e}^{\frac{z}{t}} t^{-c}\right) \mathrm{d} t
$$

Integral representations that can be derived from the representations for the confluent equation by 2nd Kummer's identity will be called Poisson-type representations. They will be treated as secondary ones. They are described in the following theorem.

Theorem 5.2. $\quad$ 1. Let the contour $\gamma$ satisfy

$$
\left.\left(t^{2}-z\right)^{-c+3 / 2} \mathrm{e}^{2 t}\right|_{\gamma(0)} ^{\gamma(1)}=0 .
$$

Then

$$
\mathcal{F}\left(c ; z, \partial_{z}\right) \int_{\gamma}\left(t^{2}-z\right)^{-c+1 / 2} \mathrm{e}^{2 t} \mathrm{~d} t=0 .
$$

2. Let the contour $\gamma$ satisfy

$$
\left.\left(t^{2}-1\right)^{c-1 / 2} \mathrm{e}^{2 t \sqrt{z}}\right|_{\gamma(0)} ^{\gamma(1)}=0 .
$$

Then

$$
\mathcal{F}\left(c ; z, \partial_{z}\right) \int_{\gamma}\left(t^{2}-1\right)^{c-3 / 2} \mathrm{e}^{2 t \sqrt{z}} \mathrm{~d} t=0 .
$$

Proof. By (5.2) and (4.4), for appropriate contours $\gamma$ and $\gamma^{\prime}$,

$$
\begin{aligned}
& \mathrm{e}^{-2 \sqrt{z}} \int_{\gamma} \mathrm{e}^{s} s^{-c+\frac{1}{2}}(s-4 \sqrt{z})^{-c+\frac{1}{2}} \mathrm{~d} s \\
& =2^{-2 c+2} \int_{\gamma^{\prime}} \mathrm{e}^{2 t}\left(t^{2}-z\right)^{-c+\frac{1}{2}} \mathrm{~d} t
\end{aligned}
$$

is annihilated by $\mathcal{F}(c)$, where we set $t=\frac{s}{2}-\sqrt{z}$. This proves 1 .

By (5.2) and (4.5), for appropriate contours $\gamma$ and $\gamma^{\prime}$,

$$
\begin{aligned}
\mathrm{e}^{-2 \sqrt{z}} & \int_{\gamma} \mathrm{e}^{\frac{4 \sqrt{z}}{s}} s^{-2 c+1}(1-s)^{c-\frac{3}{2}} \mathrm{~d} s \\
& =-2^{-2 c+2} \int_{\gamma^{\prime}} \mathrm{e}^{2 t \sqrt{z}}\left(1-t^{2}\right)^{c-\frac{3}{2}} \mathrm{~d} t
\end{aligned}
$$

is annihilated by $\mathcal{F}(c)$, where we set $t=\frac{2}{s}-1$. This proves 2 . 


\subsection{Symmetries}

The only nontrivial symmetry is

$$
z^{-\alpha} \mathcal{F}_{-\alpha} z^{\alpha}=\mathcal{F}_{\alpha}
$$

It can be interpreted as a "Weyl symmetry" of aso(2).

\subsection{Factorizations and Commutation Relations}

There are two ways to factorize the ${ }_{0} F_{1}$ operator:

$$
\begin{aligned}
\mathcal{F}_{\alpha} & =\left(z \partial_{z}+\alpha+1\right) \partial_{z}-1 \\
& =\partial_{z}\left(z \partial_{z}+\alpha\right)-1
\end{aligned}
$$

The factorizations can be used to derive the following commutation relations:

$$
\begin{aligned}
\partial_{z} & \mathcal{F}_{\alpha} \\
=\mathcal{F}_{\alpha+1} & \partial_{z}, \\
\left(z \partial_{z}+\alpha\right) & \mathcal{F}_{\alpha} \\
=\mathcal{F}_{\alpha-1} & \left(z \partial_{z}+\alpha\right) .
\end{aligned}
$$

Each commutation relation can be associated with a "root" of the Lie algebra $\operatorname{aso}(2)$.

\subsection{Canonical Forms}

The natural weight of the ${ }_{0} F_{1}$ operator is $z^{\alpha}$, so that

$$
\mathcal{F}_{\alpha}=z^{-\alpha} \partial_{z} z^{\alpha+1} \partial_{z}-1 \text {. }
$$

The balanced form of the ${ }_{0} F_{1}$ operator is

$$
z^{\frac{\alpha}{2}} \mathcal{F}_{\alpha} z^{-\frac{\alpha}{2}}=\partial_{z} z \partial_{z}-1-\frac{\alpha^{2}}{4 z} .
$$

The symmetry $\alpha \rightarrow-\alpha$ is obvious in the balanced form.

Remark 5.3. In the literature, the ${ }_{0} F_{1}$ equation is seldom used. Much more frequent is the modified Bessel equation, which is equivalent to the ${ }_{0} F_{1}$ equation:

$$
z^{\frac{\alpha}{2}} \mathcal{F}_{\alpha}\left(z, \partial_{z}\right) z^{-\frac{\alpha}{2}}=\partial_{w}^{2}+\frac{1}{w} \partial_{w}-1-\frac{\alpha^{2}}{w^{2}},
$$

where $z=\frac{w^{2}}{4}, w= \pm 2 \sqrt{z}$.

Even more frequent is the Bessel equation:

$$
-z^{\frac{\alpha}{2}} \mathcal{F}_{\alpha}\left(z, \partial_{z}\right) z^{-\frac{\alpha}{2}}=\partial_{u}^{2}+\frac{1}{u} \partial_{u}+1-\frac{\alpha^{2}}{u^{2}},
$$

where $z=-\frac{u^{2}}{4}, u= \pm 2 \mathrm{i} \sqrt{z}$. Clearly, we can pass from the modified Bessel to the Bessel equation by $w= \pm \mathrm{i} u$. 


\subsection{The ${ }_{0} F_{1}$ Function}

The ${ }_{0} F_{1}$ equation has a regular singular point at 0 . Its indices at 0 are equal to $0,1-c$.

If $c \neq 0,-1,-2, \ldots$, then the only solution of the ${ }_{0} F_{1}$ equation $\sim 1$ at 0 is called the ${ }_{0} F_{1}$ hypergeometric function. It is

$$
F(c ; z):=\sum_{j=0}^{\infty} \frac{1}{(c)_{j}} \frac{z^{j}}{j !} .
$$

It is defined for $c \neq 0,-1,-2, \ldots$ Sometimes, it is more convenient to consider the function

$$
\mathbf{F}(c ; z):=\frac{F(c ; z)}{\Gamma(c)}=\sum_{j=0}^{\infty} \frac{1}{\Gamma(c+j)} \frac{z^{j}}{j !}
$$

defined for all $c$.

We can express the ${ }_{0} F_{1}$ function in terms of the confluent function

$$
\begin{aligned}
F(c ; z) & =\mathrm{e}^{-2 \sqrt{z}} F\left(\frac{2 c-1}{2} ; 2 c-1 ; 4 \sqrt{z}\right) \\
& =\mathrm{e}^{2 \sqrt{z}} F\left(\frac{2 c-1}{2} ; 2 c-1 ;-4 \sqrt{z}\right) .
\end{aligned}
$$

It is also a limit of the confluent function.

$$
F(c ; z)=\lim _{a \rightarrow \infty} F(a ; c ; z / a) .
$$

For all parameters, we have an integral representation called the Schläfli formula:

$$
\frac{1}{2 \pi \mathrm{i}} \int_{]-\infty, 0^{+},-\infty[} \mathrm{e}^{t} \mathrm{e}^{\frac{z}{t}} t^{-c} \mathrm{~d} t=\mathbf{F}(c, z), \quad \operatorname{Re} z>0 .
$$

For $\operatorname{Re} c>\frac{1}{2}$, we have a representation called the Poisson formula:

$$
\int_{-1}^{1}\left(1-t^{2}\right)^{c-\frac{3}{2}} \mathrm{e}^{2 t \sqrt{z}}=\Gamma\left(c-\frac{1}{2}\right) \sqrt{\pi} \mathbf{F}(c, z) .
$$

We will usually prefer to use the Lie-algebraic parameters:

$$
\begin{aligned}
& F_{\alpha}(z):=F(\alpha+1 ; z), \\
& \mathbf{F}_{\alpha}(z):=\mathbf{F}(\alpha+1 ; z) .
\end{aligned}
$$

Remark 5.4. In the literature, the ${ }_{0} F_{1}$ function is seldom used. Instead, one uses the modified Bessel function and, even more frequently, the Bessel function:

$$
\begin{aligned}
& I_{\alpha}(w)=\left(\frac{w}{2}\right)^{\alpha} \mathbf{F}_{\alpha}\left(\frac{w^{2}}{4}\right), \\
& J_{\alpha}(w)=\left(\frac{w}{2}\right)^{\alpha} \mathbf{F}_{\alpha}\left(-\frac{w^{2}}{4}\right) .
\end{aligned}
$$

They solve the modified Bessel, resp. the Bessel equation. 


\subsection{Standard Solutions}

$z=0$ is a regular singular point. We have two standard solutions corresponding to its two indices. Besides, we have an additional solution with a special behavior at $\infty$.

We know from Theorem 5.1 that for appropriate contours $\gamma$ the integrals

$$
\int_{\gamma} \mathrm{e}^{t} \mathrm{e}^{\frac{z}{t}} t^{-\alpha-1} \mathrm{~d} t
$$

solve the ${ }_{0} F_{1}$ equation. The integrand goes to zero as $t \rightarrow-\infty$ and $t \rightarrow$ $0-0$ (the latter for $\operatorname{Re} z>0$ ). Therefore, contours ending at these points yield solutions. We will see that in this way, we can obtain all three standard solutions.

Besides, we can use Theorem 5.2 to obtain other integral representations, which are essentially special cases of representations for the ${ }_{1} F_{1}$ and ${ }_{2} F_{0}$ functions.

5.8.1. Solution $\sim \mathbf{1}$ at $\mathbf{0}$. If $\alpha \neq-1,-2, \ldots$, then the only solution of the ${ }_{0} F_{1}$ equation $\sim 1$ at 0 is

$$
\begin{aligned}
F_{\alpha}(z) & =\mathrm{e}^{-2 \sqrt{z}} F_{0,2 \alpha}(4 \sqrt{z}) \\
& =\mathrm{e}^{2 \sqrt{z}} F_{0,2 \alpha}(-4 \sqrt{z}) .
\end{aligned}
$$

For all parameters, we have an integral representation

$$
\frac{1}{2 \pi \mathrm{i}} \int_{]-\infty, 0^{+},-\infty[} \mathrm{e}^{t} \mathrm{e}^{\frac{z}{t}} t^{-\alpha-1} \mathrm{~d} t=\mathbf{F}_{\alpha}(z), \quad \operatorname{Re} z>0 ;
$$

and for $\operatorname{Re} \alpha>-\frac{1}{2}$, we have another integral representation

$$
\left.\left.\int_{-1}^{1}\left(1-t^{2}\right)^{\alpha-\frac{1}{2}} \mathrm{e}^{2 t \sqrt{z}} \mathrm{~d} t=\Gamma\left(\alpha+\frac{1}{2}\right) \sqrt{\pi} \mathbf{F}_{\alpha}(z), \quad z \notin\right]-\infty, 0\right] .
$$

5.8.2. Solution $\sim \boldsymbol{z}^{-\boldsymbol{\alpha}}$ at 0 . If $\alpha \neq 1,2, \ldots$, then the only solution to the ${ }_{0} F_{1}$ equation $\sim z^{-\alpha}$ at 0 is

$$
\begin{aligned}
z^{-\alpha} F_{-\alpha}(z) & =z^{-\alpha} \mathrm{e}^{-2 \sqrt{z}} F_{0,-2 \alpha}(4 \sqrt{z}) \\
& =z^{-\alpha} \mathrm{e}^{2 \sqrt{z}} F_{0,-2 \alpha}(-4 \sqrt{z}) .
\end{aligned}
$$

For all parameters, we have

$$
\frac{1}{2 \pi \mathrm{i}} \int_{\left[(0-0)^{+}\right]} \mathrm{e}^{t} \mathrm{e}^{\frac{z}{t}} t^{-\alpha-1} \mathrm{~d} t=z^{-\alpha} \mathbf{F}_{-\alpha}(z), \quad \operatorname{Re} z>0
$$

and for $\frac{1}{2}>\alpha$, we have

$$
\left.\left.\int_{-\sqrt{z}}^{\sqrt{z}}\left(z-t^{2}\right)^{-\alpha-\frac{1}{2}} \mathrm{e}^{2 t} \mathrm{~d} t=\Gamma\left(-\alpha+\frac{1}{2}\right) \sqrt{\pi} z^{-\alpha} \mathbf{F}_{-\alpha}(z), \quad z \notin\right]-\infty, 0\right] .
$$


5.8.3. Solution $\sim \exp \left(-2 z^{\frac{1}{2}}\right) z^{-\frac{\alpha}{2}-\frac{1}{4}}$ for $z \rightarrow+\infty$. The following function is also a solution of the ${ }_{0} F_{1}$ equation:

$$
\tilde{F}_{\alpha}(z):=\mathrm{e}^{-2 \sqrt{z}} z^{-\frac{\alpha}{2}-\frac{1}{4}} \tilde{F}_{0,2 \alpha}\left(-\frac{1}{4 \sqrt{z}}\right) .
$$

We have the identity

$$
\tilde{F}_{\alpha}(z)=z^{-\alpha} \tilde{F}_{-\alpha}(z) .
$$

Integral representations for all parameters:

$$
\int_{-\infty}^{0} \mathrm{e}^{t} \mathrm{e}^{\frac{z}{t}}(-t)^{-\alpha-1} \mathrm{~d} t=\pi^{\frac{1}{2}} \tilde{F}_{\alpha}(z), \quad \operatorname{Re} z>0
$$

for $\operatorname{Re} \alpha>-\frac{1}{2}$ :

$$
\left.\left.\int_{-\infty}^{-1}\left(t^{2}-1\right)^{\alpha-\frac{1}{2}} \mathrm{e}^{2 t \sqrt{z}} \mathrm{~d} t=\frac{1}{2} \Gamma\left(\alpha+\frac{1}{2}\right) \tilde{F}_{\alpha}(z), \quad z \notin\right]-\infty, 0\right] ;
$$

for $\operatorname{Re} \alpha<\frac{1}{2}$ :

$$
\left.\left.\int_{-\infty}^{-\sqrt{z}}\left(t^{2}-z\right)^{-\alpha-\frac{1}{2}} \mathrm{e}^{2 t} \mathrm{~d} t=\frac{1}{2} \Gamma\left(-\alpha+\frac{1}{2}\right) \tilde{F}_{\alpha}(z), \quad z \notin\right]-\infty, 0\right] .
$$

As $|z| \rightarrow \infty$ and $|\arg z|<\pi / 2-\epsilon$, we have

$$
\tilde{F}_{\alpha}(z) \sim \exp \left(-2 z^{\frac{1}{2}}\right) z^{-\frac{\alpha}{2}-\frac{1}{4}} .
$$

$F_{\alpha}$ is a unique solution with this property.

To prove (5.5), we can use the saddle point method. We write the left hand side as

$$
\int_{0}^{\infty} \mathrm{e}^{\phi(s)} s^{-\alpha-1} \mathrm{~d} s
$$

with $\phi(s)=-s-\frac{z}{s}$. We compute:

$$
\phi^{\prime}(s)=-1+\frac{z}{s^{2}}, \quad \phi^{\prime \prime}(s)=-2 \frac{z}{s^{3}} .
$$

We find the stationary point at $s_{0}=\sqrt{z}$ with $\phi^{\prime \prime}\left(s_{0}\right)=-2 z^{-\frac{1}{2}}$ and $\phi\left(s_{0}\right)=$ $-2 \sqrt{z}$. Hence, the left hand side of $(5.5)$ can be approximated by

$$
\int_{-\infty}^{\infty} \mathrm{e}^{\phi\left(s_{0}\right)+\frac{1}{2}\left(s-s_{0}\right)^{2} \phi^{\prime \prime}\left(s_{0}\right)} s_{0}^{-\alpha-1} \mathrm{~d} s=\pi^{\frac{1}{2}} z^{-\frac{\alpha}{2}-\frac{1}{4}} \mathrm{e}^{-2 \sqrt{z}} .
$$

Remark 5.5. In the literature, instead of the $\tilde{F}$ function one uses the MacDonald function, solving the modified Bessel equation:

$$
K_{\alpha}(w)=\sqrt{\pi}\left(\frac{w}{2}\right)^{\alpha} \tilde{F}_{\alpha}\left(\frac{w^{2}}{4}\right)
$$


and the Hankel functions of the 1st and 2nd kind, solving the Bessel equation:

$$
\begin{aligned}
& H_{\alpha}^{(1)}(w)=\frac{\mathrm{i}}{\sqrt{\pi}}\left(\frac{\mathrm{e}^{-\mathrm{i} \pi / 2} w}{2}\right)^{\alpha} \tilde{F}_{\alpha}\left(\mathrm{e}^{-\mathrm{i} \pi} \frac{w^{2}}{4}\right), \\
& H_{\alpha}^{(2)}(w)=-\frac{\mathrm{i}}{\sqrt{\pi}}\left(\frac{\mathrm{e}^{\mathrm{i} \pi / 2} w}{2}\right)^{\alpha} \tilde{F}_{\alpha}\left(\mathrm{e}^{\mathrm{i} \pi} \frac{w^{2}}{4}\right) .
\end{aligned}
$$

\subsection{Connection Formulas}

We can use the solutions with a simple behavior at zero as the basis:

$$
\tilde{F}_{\alpha}(z)=\frac{\sqrt{\pi}}{\sin \pi(-\alpha)} \mathbf{F}_{\alpha}(z)+\frac{\sqrt{\pi}}{\sin \pi \alpha} z^{-\alpha} \mathbf{F}_{-\alpha}(z) .
$$

Alternatively, we can use the $\tilde{F}$ function and its analytic continuation around 0 in the clockwise or anti-clockwise direction as the basis:

$$
\begin{aligned}
\mathbf{F}_{\alpha}(z) & =\frac{1}{2 \pi^{\frac{3}{2}}}\left(\mathrm{e}^{-\mathrm{i} \pi\left(\alpha-\frac{1}{2}\right)} \tilde{F}_{\alpha}(z)-\mathrm{e}^{\mathrm{i} \pi\left(\alpha-\frac{1}{2}\right)} \tilde{F}_{\alpha}\left(\mathrm{e}^{-\mathrm{i} 2 \pi} z\right)\right) \\
& =\frac{1}{2 \pi^{\frac{3}{2}}}\left(\mathrm{e}^{-\mathrm{i} \pi\left(\alpha-\frac{1}{2}\right)} \tilde{F}_{\alpha}\left(\mathrm{e}^{\mathrm{i} 2 \pi} z\right)-\mathrm{e}^{\mathrm{i} \pi\left(\alpha-\frac{1}{2}\right)} \tilde{F}_{\alpha}(z)\right), \\
z^{-\alpha} \mathbf{F}_{-\alpha}(z) & =\frac{1}{2 \pi^{\frac{3}{2}}}\left(\mathrm{e}^{\mathrm{i} \pi\left(\alpha+\frac{1}{2}\right)} \tilde{F}_{\alpha}(z)-\mathrm{e}^{-\mathrm{i} \pi\left(\alpha+\frac{1}{2}\right)} \tilde{F}_{\alpha}\left(\mathrm{e}^{-\mathrm{i} 2 \pi} z\right)\right) \\
& =\frac{1}{2 \pi^{\frac{3}{2}}}\left(\mathrm{e}^{\mathrm{i} \pi\left(\alpha+\frac{1}{2}\right)} \tilde{F}_{\alpha}\left(\mathrm{e}^{\mathrm{i} 2 \pi} z\right)-\mathrm{e}^{-\mathrm{i} \pi\left(\alpha+\frac{1}{2}\right)} \tilde{F}_{\alpha}(z)\right) .
\end{aligned}
$$

\subsection{Recurrence Relations}

The following recurrence relations easily follow from the commutation relations of Sect. 5.5:

$$
\begin{aligned}
\partial_{z} \mathbf{F}_{\alpha}(z) & =\mathbf{F}_{\alpha+1}(z), \\
\left(z \partial_{z}+\alpha\right) \mathbf{F}_{\alpha}(z) & =\mathbf{F}_{\alpha-1}(z) .
\end{aligned}
$$

\subsection{Degenerate Case}

$\alpha=m \in \mathbb{Z}$ is the degenerate case of the ${ }_{0} F_{1}$ equation at 0 . We have then

$$
\mathbf{F}(1+m ; z)=\sum_{n=\max (0,-m)} \frac{1}{n !(m+n) !} z^{n} .
$$

This easily implies the identity

$$
\mathbf{F}(1+m ; z)=z^{-m} \mathbf{F}(1-m ; z) .
$$

Thus, the two standard solutions determined by the behavior at zero are proportional to one another.

We have an integral representation, called the Bessel formula, and a generating function:

$$
\begin{aligned}
\frac{1}{2 \pi i} \int_{\left[0^{+}\right]} \mathrm{e}^{t+z / t} t^{-m-1} \mathrm{~d} t & =\mathbf{F}_{m}(z)=z^{-m} \mathbf{F}_{-m}(z), \\
\mathrm{e}^{t} \mathrm{e}^{z / t} & =\sum_{m \in \mathbb{Z}} t^{m} \mathbf{F}_{m}(z) .
\end{aligned}
$$




\subsection{Special Cases}

If $\alpha= \pm \frac{1}{2}$, then the ${ }_{0} F_{1}$ equation can be reduced to an equation easily solvable in terms of elementary functions:

$$
\begin{aligned}
\mathcal{F}_{-\frac{1}{2}}\left(z, \partial_{z}\right) & =\partial_{u}^{2}-1, \\
\mathcal{F}_{\frac{1}{2}}\left(z, \partial_{z}\right) & =u^{-1}\left(\partial_{u}^{2}-1\right) u,
\end{aligned}
$$

where $u=2 \sqrt{z}$. They have solutions

$$
\begin{array}{cl}
F_{-\frac{1}{2}}(z)=\cosh 2 \sqrt{z}, & \tilde{F}_{-\frac{1}{2}}(z)=\exp (-2 \sqrt{z}), \\
F_{\frac{1}{2}}(z)=\frac{\sinh 2 \sqrt{z}}{2 \sqrt{z}}, & \tilde{F}_{\frac{1}{2}}(z)=\frac{\exp (-2 \sqrt{z})}{\sqrt{z}} .
\end{array}
$$

\section{The Gegenbauer Equation}

\subsection{Introduction}

The hypergeometric equation can be moved by an affine transformation so that its finite singular points are placed at -1 and 1 . If in addition the equation is reflection invariant, then it will be called the Gegenbauer equation.

Because of the reflection invariance, the third classical parameter can be obtained from the first two: $c=\frac{a+b+1}{2}$. Therefore, we will use only $a, b \in \mathbb{C}$ as the (classical) parameters of the Gegenbauer equation. It will be given by the operator

$$
\mathcal{S}\left(a, b ; z, \partial_{z}\right):=\left(1-z^{2}\right) \partial_{z}^{2}-(a+b+1) z \partial_{z}-a b .
$$

To describe the symmetries of the Gegenbauer operator, it is convenient to use its Lie-algebraic parameters

$$
\begin{aligned}
\alpha:=\frac{a+b-1}{2}, & \lambda:=\frac{b-a}{2}, \\
a=\frac{1}{2}+\alpha-\lambda, & b=\frac{1}{2}+\alpha+\lambda .
\end{aligned}
$$

Thus, (6.1) becomes

$$
\mathcal{S}_{\alpha, \lambda}\left(z, \partial_{z}\right):=\left(1-z^{2}\right) \partial_{z}^{2}-2(1+\alpha) z \partial_{z}+\lambda^{2}-\left(\alpha+\frac{1}{2}\right)^{2} .
$$

The Lie-algebraic parameters have an interesting interpretation in terms of the natural basis of the Cartan algebra of the Lie algebra so(5) [2].

\subsection{Equivalence with the Hypergeometric Equation}

The Gegenbauer equation is equivalent to certain subclasses of the hypergeometric equation by a number of different substitutions.

First of all, we can reduce the Gegenbauer equation to the hypergeometric equation by two affine transformations. They move the singular points from $-1,1$ to 0,1 or 1,0 :

$$
\mathcal{S}\left(a, b ; z, \partial_{z}\right)=\mathcal{F}\left(a, b ; \frac{a+b+1}{2} ; u, \partial_{u}\right),
$$


where

$$
\begin{aligned}
u & =\frac{1-z}{2}, \quad z=1-2 u, \\
\text { or } \quad u & =\frac{1+z}{2}, \quad z=-1+2 u .
\end{aligned}
$$

In the Lie-algebraic parameters

$$
\mathcal{S}_{\alpha, \lambda}\left(z, \partial_{z}\right)=\mathcal{F}_{\alpha, \alpha, 2 \lambda}\left(u, \partial_{u}\right) .
$$

Another pair of substitutions is a consequence of the reflection invariance of the Gegenbauer equation (see Sect. 2.4):

$$
\begin{aligned}
\mathcal{S}\left(a, b ; z, \partial_{z}\right) & =4 \mathcal{F}\left(\frac{a}{2}, \frac{b}{2} ; \frac{1}{2} ; w, \partial_{w}\right), \\
z^{-1} \mathcal{S}\left(a, b ; z, \partial_{z}\right) z & =4 \mathcal{F}\left(\frac{a+1}{2}, \frac{b+1}{2} ; \frac{3}{2} ; w, \partial_{w}\right),
\end{aligned}
$$

where

$$
w=z^{2}, \quad z=\sqrt{w}
$$

In the Lie-algebraic parameters

$$
\begin{aligned}
\mathcal{S}_{\alpha, \lambda}\left(z, \partial_{z}\right) & =\mathcal{F}_{-\frac{1}{2}, \alpha, \lambda}\left(w, \partial_{w}\right) \\
z^{-1} \mathcal{S}_{\alpha, \lambda}\left(z, \partial_{z}\right) z & =\mathcal{F}_{\frac{1}{2}, \alpha, \lambda}\left(w, \partial_{w}\right)
\end{aligned}
$$

\subsection{Symmetries}

All the operators below equal $\mathcal{S}_{\alpha, \lambda}\left(w, \partial_{w}\right)$ for an appropriate $w$ :

$$
\begin{aligned}
& w= \pm z: \\
& w= \pm z: \\
& \mathcal{S}_{\alpha, \pm \lambda} \\
& \left(z^{2}-1\right)^{-\alpha} \mathcal{S}_{-\alpha, \pm \lambda}\left(z^{2}-1\right)^{\alpha} \\
& w=\frac{ \pm z}{\left(z^{2}-1\right)^{\frac{1}{2}}}: \\
& w=\frac{ \pm z}{\left(z^{2}-1\right)^{\frac{1}{2}}}: \\
& \left(z^{2}-1\right)^{\frac{1}{2}\left(\alpha+\lambda+\frac{5}{2}\right)} \quad \mathcal{S}_{\lambda, \pm \alpha} \quad\left(z^{2}-1\right)^{\frac{1}{2}\left(-\alpha-\lambda-\frac{1}{2}\right)}, \\
& \left(z^{2}-1\right)^{\frac{1}{2}\left(\alpha-\lambda+\frac{5}{2}\right)} \quad \mathcal{S}_{-\lambda, \pm \alpha} \quad\left(z^{2}-1\right)^{\frac{1}{2}\left(-\alpha+\lambda-\frac{1}{2}\right)} .
\end{aligned}
$$

The symmetries of the Gegenbauer operator have an interpretation in terms of the Weyl group of the Lie algebra so(5).

Note that the first two symmetries from the above table are inherited from the hypergeometric equation through the substitution (6.2).

The symmetries involving $w=\frac{ \pm z}{\left(z^{2}-1\right)^{\frac{1}{2}}}$ go under the name of the Whipple transformation. To obtain them, we first use the substitution (6.3) $z \rightarrow z^{2}$, then $z^{2} \rightarrow \frac{z^{2}}{1-z^{2}}$, which is one of the symmetries from the Kummer's table, finally the substitution (6.3) in the opposite direction $\frac{z^{2}}{1-z^{2}} \rightarrow \sqrt{\frac{z^{2}}{1-z^{2}}}$. We will continue our discussion of the Whipple transformation in Sect. 6.5. 


\subsection{Factorizations and Commutation Relations}

There are several ways of factorizing the Gegenbauer operator:

$$
\begin{aligned}
& \mathcal{S}_{\alpha, \lambda}=\left(\left(1-z^{2}\right) \partial_{z}-2(1+\alpha) z\right) \partial_{z} \\
& +\left(\alpha+\lambda+\frac{1}{2}\right)\left(-\alpha+\lambda-\frac{1}{2}\right) \\
& =\partial_{z}\left(\left(1-z^{2}\right) \partial_{z}-2 \alpha z\right) \\
& +\left(\alpha+\lambda-\frac{1}{2}\right)\left(-\alpha+\lambda+\frac{1}{2}\right), \\
& \left(1-z^{2}\right) \mathcal{S}_{\alpha, \lambda}=\left(\left(1-z^{2}\right) \partial_{z}+\left(\alpha-\lambda+\frac{3}{2}\right) z\right)\left(\left(1-z^{2}\right) \partial_{z}+\left(\alpha+\lambda+\frac{1}{2}\right) z\right) \\
& -\left(\alpha+\lambda+\frac{1}{2}\right)\left(\alpha-\lambda+\frac{3}{2}\right) \\
& =\left(\left(1-z^{2}\right) \partial_{z}+\left(\alpha+\lambda+\frac{3}{2}\right) z\right)\left(\left(1-z^{2}\right) \partial_{z}+\left(\alpha-\lambda+\frac{1}{2}\right) z\right) \\
& -\left(\alpha-\lambda+\frac{1}{2}\right)\left(\alpha+\lambda+\frac{3}{2}\right) \text {; } \\
& z^{2} \mathcal{S}_{\alpha, \lambda}=\left(z\left(1-z^{2}\right) \partial_{z}-\alpha-\lambda-\frac{3}{2}+\left(-\alpha+\lambda-\frac{1}{2}\right) z^{2}\right)\left(z \partial_{z}+\alpha+\lambda+\frac{1}{2}\right) \\
& +\left(\alpha+\lambda+\frac{1}{2}\right)\left(\alpha+\lambda+\frac{3}{2}\right) \\
& =\left(z \partial_{z}+\alpha+\lambda-\frac{3}{2}\right)\left(z\left(1-z^{2}\right) \partial_{z}-\alpha-\lambda+\frac{1}{2}+\left(-\alpha+\lambda-\frac{1}{2}\right) z^{2}\right) \\
& +\left(\alpha+\lambda-\frac{1}{2}\right)\left(\alpha+\lambda-\frac{3}{2}\right) \\
& =\left(z\left(1-z^{2}\right) \partial_{z}-\alpha+\lambda-\frac{3}{2}+\left(-\alpha-\lambda-\frac{1}{2}\right) z^{2}\right)\left(z \partial_{z}+\alpha-\lambda+\frac{1}{2}\right) \\
& +\left(\alpha-\lambda+\frac{1}{2}\right)\left(\alpha-\lambda+\frac{3}{2}\right) \\
& =\left(z \partial_{z}+\alpha-\lambda-\frac{3}{2}\right)\left(z\left(1-z^{2}\right) \partial_{z}-\alpha+\lambda+\frac{1}{2}+\left(-\alpha-\lambda-\frac{1}{2}\right) z^{2}\right) \\
& +\left(\alpha-\lambda-\frac{1}{2}\right)\left(\alpha-\lambda-\frac{3}{2}\right) \text {. }
\end{aligned}
$$

The following commutation relations can be derived from the factorizations:

$$
\begin{aligned}
\partial_{z} & \mathcal{S}_{\alpha, \lambda} \\
=\mathcal{S}_{\alpha+1, \lambda} & \partial_{z} \\
=\partial_{\alpha}-1, \lambda & \mathcal{S}_{\alpha, \lambda} \\
\left(\left(1-z^{2}\right)\right. & \left.\left(1-z^{2}\right) \partial_{z}-2 \alpha z\right) \\
\left(\left(1-z^{2}\right) \partial_{z}-\left(\alpha+\lambda+\frac{1}{2}\right) z\right) & \left(1-z^{2}\right) \mathcal{S}_{\alpha, \lambda} \\
=\left(1-z^{2}\right) \mathcal{S}_{\alpha, \lambda+1} & \left(\left(1-z^{2}\right) \partial_{z}-\left(\alpha+\lambda+\frac{1}{2}\right) z\right) \\
\left(\left(1-z^{2}\right) \partial_{z}-\left(\alpha-\lambda+\frac{1}{2}\right) z\right) & \left(1-z^{2}\right) \mathcal{S}_{\alpha, \lambda} \\
=\left(1-z^{2}\right) \mathcal{S}_{\alpha, \lambda-1} & \left(\left(1-z^{2}\right) \partial_{z}-\left(\alpha-\lambda+\frac{1}{2}\right) z\right) \\
\left(z \partial_{z}+\alpha-\lambda+\frac{1}{2}\right) & z^{2} \mathcal{S}_{\alpha, \lambda} \\
=z^{2} \mathcal{S}_{\alpha+1, \lambda-1} & \left(z \partial_{z}+\alpha-\lambda+\frac{1}{2}\right)
\end{aligned}
$$




$$
\begin{aligned}
\left(z\left(1-z^{2}\right) \partial_{z}-\alpha+\lambda+\frac{1}{2}-\left(\alpha+\lambda+\frac{1}{2}\right) z^{2}\right) & z^{2} \mathcal{S}_{\alpha, \lambda} \\
=z^{2} \mathcal{S}_{\alpha-1, \lambda+1} & \left(z\left(1-z^{2}\right) \partial_{z}-\alpha+\lambda+\frac{1}{2}-\left(\alpha+\lambda+\frac{1}{2}\right) z^{2}\right), \\
\left(z \partial_{z}+\alpha+\lambda+\frac{1}{2}\right) & z^{2} \mathcal{S}_{\alpha, \lambda} \\
=z^{2} \mathcal{S}_{\alpha+1, \lambda+1} & \left(z \partial_{z}+\alpha+\lambda+\frac{1}{2}\right) \\
\left(z\left(1-z^{2}\right) \partial_{z}-\alpha-\lambda+\frac{1}{2}-\left(\alpha-\lambda+\frac{1}{2}\right) z^{2}\right) & z^{2} \mathcal{S}_{\alpha, \lambda} \\
=z^{2} \mathcal{S}_{\alpha-1, \lambda-1} & \left(z\left(1-z^{2}\right) \partial_{z}-\alpha-\lambda+\frac{1}{2}-\left(\alpha-\lambda+\frac{1}{2}\right) z^{2}\right) .
\end{aligned}
$$

Each of these commutation relations is associated with a root of the Lie algebra so(5).

Note that only the first pair of commutation relations is directly inherited from the basic commutation relations of the hypergeometric equation of Sect. 3.4. The next pair comes from what we called additional commutation relations (see Sect. 3.10), which in the reflection invariant case simplify, so that they can be counted as basic commutation relations (see a discussion in Sect. 1.2). Note that the Whipple transformation transforms the first pair of the commutation relations into the second, and the other way around.

The last four commutation relations form a separate class - they can be obtained by applying consecutively an appropriate pair from the first four commutation relations.

\subsection{The Riemann Surface of the Gegenbauer Equation}

Let us analyze more closely the Whipple symmetry.

First let us precise the meaning of the holomorphic function involved in this symmetry. If $z \in \Omega_{+}:=\mathbb{C} \backslash[-1,1]$, then $\left.\left.1-z^{-2} \in \mathbb{C} \backslash\right]-\infty, 0\right]$. Therefore,

$$
\frac{z}{\left(z^{2}-1\right)^{\frac{1}{2}}}:=\frac{1}{\left(1-z^{-2}\right)^{\frac{1}{2}}}
$$

defines a unique analytic function on $z \in \Omega_{+}$(where on the right we have the principal branch of the square root). Note that, for $z \rightarrow \infty,(6.6)$ converges to 1 .

Consider a second copy of $\Omega_{+}$, denoted $\Omega_{-}$. Glue them together along ] $-1,1\left[\right.$, so that crossing ] $-1,1\left[\right.$ we go from $\Omega_{ \pm}$to $\Omega_{\mp}$. The resulting complex manifold will be called $\Omega$. The elements of $\Omega_{ \pm}$corresponding to $\left.z \in \mathbb{C} \backslash\right]-1,1[$ will be denoted $z_{ \pm} . \Omega$ is biholomorphic to the sphere with 4 punctures, which correspond to the points $-1,1, \infty_{+}, \infty_{-}$.

It is easy to see that $\Omega$ is the Riemann surface of the maximal holomorphic function extending (6.6). On $\Omega_{-}$it equals $-\frac{z}{\left(z^{2}-1\right)^{\frac{1}{2}}}$.

It is useful to reinterpret this holomorphic function as a biholomorphic function from $\Omega$ into itself:

$$
\begin{gathered}
\tau\left(z_{+}\right):= \begin{cases}\left(\frac{z}{\sqrt{z^{2}-1}}\right)_{+}, & \operatorname{Re} z>0, \\
\left(\frac{z}{\sqrt{z^{2}-1}}\right)_{-}, & \operatorname{Re} z<0,\end{cases} \\
\tau\left(z_{-}\right):= \begin{cases}\left(-\frac{z}{\sqrt{z^{2}-1}}\right)_{-}, & \operatorname{Re} z>0, \\
\left(-\frac{z}{\sqrt{z^{2}-1}}\right)_{+}, & \operatorname{Re} z<0 .\end{cases}
\end{gathered}
$$


We also introduce

$$
\begin{aligned}
\epsilon\left(z_{ \pm}\right) & :=z_{\mp}, \\
(-1) z_{ \pm} & :=(-z)_{ \pm} .
\end{aligned}
$$

Note that $\tau^{2}=\mathrm{id}, \epsilon^{2}=\mathrm{id},(-1)^{2}=\mathrm{id}, \tau \epsilon=(-1) \epsilon \tau . \tau$ and $\epsilon$ generate a group isomorphic to the group of the symmetries of the square. The vertices of this square can be identified with $\left(1, \infty_{+},-1, \infty_{-}\right)$. They are permuted by these transformations as follows:

$$
\begin{aligned}
\epsilon\left(1, \infty_{+},-1, \infty_{-}\right) & =\left(1, \infty_{-},-1, \infty_{+}\right), \\
(-1)\left(1, \infty_{+},-1, \infty_{-}\right) & =\left(-1, \infty_{+}, 1, \infty_{-}\right), \\
\tau\left(1, \infty_{+},-1, \infty_{-}\right) & =\left(\infty_{+}, 1, \infty_{-},-1\right) .
\end{aligned}
$$

It is useful to view the Gegenbauer equation as defined on $\Omega$.

\subsection{Integral Representations}

Theorem 6.1. 1. Let $[0,1] \ni t \mapsto \gamma(t)$ satisfy

$$
\left.\left(t^{2}-1\right)^{\frac{b-a+1}{2}}(t-z)^{-b-1}\right|_{\gamma(0)} ^{\gamma(1)}=0
$$

Then

$$
\mathcal{S}\left(a, b ; z, \partial_{z}\right) \int_{\gamma}\left(t^{2}-1\right)^{\frac{b-a-1}{2}}(t-z)^{-b} \mathrm{~d} t=0 .
$$

2. Let $[0,1] \ni t \mapsto \gamma(t)$ satisfy

$$
\left.\left(t^{2}+2 t z+1\right)^{\frac{-b-a}{2}+1} t^{b-2}\right|_{\gamma(0)} ^{\gamma(1)}=0 .
$$

Then

$$
\mathcal{S}\left(a, b ; z, \partial_{z}\right) \int_{\gamma}\left(t^{2}+2 t z+1\right)^{\frac{-b-a}{2}} t^{b-1} \mathrm{~d} t=0 .
$$

Proof. We compute that (6.7) and (6.8) equal

$$
\begin{gathered}
a \int_{\gamma}\left(\partial_{t}\left(t^{2}-1\right)^{\frac{b-a+1}{2}}(t-z)^{-b-1}\right) \mathrm{d} t, \\
\int_{\gamma}\left(\partial_{t}\left(t^{2}+2 t z+1\right)^{\frac{-b-a}{2}+1} t^{b-2}\right) \mathrm{d} t
\end{gathered}
$$

respectively.

Note that (6.7) is essentially a special case of Theorem 3.1.

(6.8) can be derived from (6.7). In fact, using the Whipple symmetry we see that, for an appropriate contour $\tilde{\gamma}$,

$$
\left(z^{2}-1\right)^{-\frac{a}{2}} \int_{\tilde{\gamma}}\left(s^{2}-1\right)^{\frac{-b-a}{2}}\left(s-\frac{z}{\sqrt{z^{2}-1}}\right)^{b-1} \mathrm{~d} s
$$


solves the Gegenbauer equation. Then, we change the variables

$$
t=s \sqrt{z^{2}-1}-z, \quad s=\frac{t+z}{\sqrt{z^{2}-1}},
$$

and we obtain that (6.9) equals

$$
\int_{\gamma}\left(t^{2}+2 t z+1\right)^{\frac{-b-a}{2}} t^{b-1} \mathrm{~d} t
$$

with an appropriate contour $\gamma$.

Note that in the above theorem, we can interchange $a$ and $b$. Thus, we obtain four kinds of integral representations.

\subsection{Canonical Forms}

The natural weight of the Gegenbauer operator equals $\left(z^{2}-1\right)^{\alpha}$, so that

$$
\mathcal{S}_{\alpha, \lambda}=-\left(z^{2}-1\right)^{-\alpha} \partial_{z}\left(z^{2}-1\right)^{\alpha+1} \partial_{z}+\lambda^{2}-\left(\alpha+\frac{1}{2}\right)^{2} .
$$

The balanced form of the Gegenbauer operator is

$$
\begin{aligned}
& \left(z^{2}-1\right)^{\frac{\alpha}{2}} \mathcal{S}_{\alpha, \lambda}\left(z^{2}-1\right)^{-\frac{\alpha}{2}} \\
& \quad=\partial_{z}\left(1-z^{2}\right) \partial_{z}-\frac{\alpha^{2}}{1-z^{2}}+\lambda^{2}-\frac{1}{4} .
\end{aligned}
$$

Note that the symmetries $\alpha \rightarrow-\alpha$ and $\lambda \rightarrow-\lambda$ are obvious in the balanced form.

Remark 6.2. In the literature, the Gegenbauer equation is used mostly in the context of Gegenbauer polynomials, that is for $-a=0,1,2, \ldots$ In the general case, instead of the Gegenauer equation one usually considers the socalled associated Legendre equation. It coincides with the balanced form of the Gegenbauer equation, except that one of its parameters is shifted by $\frac{1}{2}$. In the standard form it is

$$
\left(1-z^{2}\right) \partial_{z}^{2}-2 z \partial_{z}-\frac{m^{2}}{1-z^{2}}+l(l+1),
$$

so that $m, l$ correspond to $\alpha, \lambda-\frac{1}{2}$ according to our convention.

\subsection{Even Solution}

Inserting a power series into equation, we see that the Gegenbauer equation possesses an even solution equal to

$$
\begin{aligned}
S_{\alpha, \lambda}^{+}(z) & :=\sum_{j=0}^{\infty} \frac{\left(\frac{a}{2}\right)_{j}\left(\frac{b}{2}\right)_{j}}{(2 j) !}(2 z)^{2 j} \\
& =F\left(\frac{a}{2}, \frac{b}{2} ; \frac{1}{2} ; z^{2}\right)=F_{-\frac{1}{2}, \alpha, \lambda}\left(z^{2}\right)
\end{aligned}
$$

It is the unique solution of the Gegenbauer equation satisfying

$$
S_{\alpha, \lambda}^{+}(0)=1, \quad \frac{\mathrm{d}}{\mathrm{d} z} S_{\alpha, \lambda}^{+}(0)=0 .
$$


One way to derive the expression in terms of the hypergeometric function is to use the transformation (6.3).

We have the identities

$$
\begin{aligned}
S_{\alpha, \lambda}^{+}(z) & =\left(1-z^{2}\right)^{\frac{-1-2 \alpha \pm 2 \lambda}{4}} S_{\mp \lambda, \alpha}^{+}\left(\frac{\mathrm{i} z}{\sqrt{1-z^{2}}}\right) \\
& =\left(1-z^{2}\right)^{-\alpha} S_{-\alpha, \lambda}^{+}(z),
\end{aligned}
$$

beside the obvious ones

$$
S_{\alpha, \lambda}^{+}(z)=S_{\alpha,-\lambda}^{+}(z)=S_{\alpha, \lambda}^{+}(-z)=S_{\alpha,-\lambda}^{+}(-z),
$$

\subsection{Odd Solution}

Similarly, the Gegenbauer equation possesses an odd solution equal to

$$
\begin{aligned}
S_{\alpha, \lambda}^{-}(z) & :=\sum_{j=0}^{\infty} \frac{\left(\frac{a+1}{2}\right)_{j}\left(\frac{b+1}{2}\right)_{j}}{(2 j+1) !}(2 z)^{2 j+1} \\
& =2 z F\left(\frac{a+1}{2}, \frac{b+1}{2} ; \frac{3}{2} ; z^{2}\right)=2 z F_{\frac{1}{2}, \alpha, \lambda}\left(z^{2}\right) .
\end{aligned}
$$

It is the unique solution of the Gegenbauer equation satisfying

$$
S_{\alpha, \lambda}^{-}(0)=0, \quad \frac{\mathrm{d}}{\mathrm{d} z} S_{\alpha, \lambda}^{-}(0)=2 .
$$

We have the identities

$$
\begin{aligned}
S_{\alpha, \lambda}^{-}(z) & =-\mathrm{i}\left(1-z^{2}\right)^{\frac{-1-2 \alpha \pm 2 \lambda}{4}} S_{\mp \lambda, \alpha}^{-}\left(\frac{\mathrm{i} z}{\sqrt{1-z^{2}}}\right) \\
& =\left(1-z^{2}\right)^{-\alpha} S_{-\alpha, \lambda}^{-}(z),
\end{aligned}
$$

beside the obvious ones:

$$
S_{\alpha, \lambda}^{+}(z)=S_{\alpha,-\lambda}^{+}(z)=-S_{\alpha, \lambda}^{+}(-z)=-S_{\alpha,-\lambda}^{+}(-z),
$$

\subsection{Standard Solutions}

As usual, by standard solutions, we mean solutions with a simple behavior around singular points. The singular points of the Gegenbauer equation are at $\{1,-1, \infty\}$. The discussion of the point -1 can be easily reduced to that of 1 . Therefore, it is enough to discuss $2 \times 2$ solutions corresponding to two indices at 1 and $\infty$.

By Theorem 6.1, for appropriate $\gamma_{1}, \gamma_{2}$ the integrals

$$
\begin{array}{r}
\int_{\gamma_{1}}\left(t^{2}-1\right)^{-\frac{1}{2}+\lambda}(t-z)^{-\frac{1}{2}-\alpha-\lambda} \mathrm{d} t, \\
\int_{\gamma_{2}}\left(t^{2}+2 t z+1\right)^{-\alpha-\frac{1}{2}}(-t)^{-\frac{1}{2}+\alpha+\lambda} \mathrm{d} t
\end{array}
$$

are solutions.

The natural endpoints of $\gamma_{1}$ are $-1,1, z, \infty$. We will see that all standard solutions can be obtained from such integrals. 
The natural endpoints of $\gamma_{2}$ are $z+\sqrt{z^{2}-1}, z-\sqrt{z^{2}-1}, 0, \infty$. Similarly, all standard solutions can be obtained from the integrals over contours with these endpoints.

It is interesting to note that in some aspects the theory of the Gegenbauer equation is more complicated than that of the hypergeometric equation. One of its manifestations is a relatively big number of natural normalizations of solutions. Indeed, let us consider, e.g., integral representations of the type (6.12). The natural endpoints fall into two categories: $\{1,-1\}$ and $\{0, \infty\}$. Therefore, we have three kinds of contours joining two of these endpoint: $[-1,1],[0, \infty[$ and the contours joining two distinct categories. This corresponds two three distinct natural normalizations, which we describe in what follows.

6.10.1. Solution $\sim 1$ at 1 . If $\alpha \neq-1,-2, \ldots$, then the unique solution of the Gegenbauer equation equal to 1 at 1 is the following function:

$$
\begin{aligned}
S_{\alpha, \lambda}(z) & :=F_{\alpha, \alpha, 2 \lambda}\left(\frac{1-z}{2}\right)=F\left(a, b ; \frac{a+b+1}{2} ; \frac{1-z}{2}\right) \\
& =F_{\alpha,-\frac{1}{2}, \lambda}\left(1-z^{2}\right)=F\left(\frac{a}{2}, \frac{b}{2} ; \frac{a+b+1}{2} ; 1-z^{2}\right) .
\end{aligned}
$$

We will also introduce several alternatively normalized functions:

$$
\begin{aligned}
\mathbf{S}_{\alpha, \lambda}(z) & :=\frac{1}{\Gamma(\alpha+1)} S_{\alpha, \lambda}(z) \\
& =\frac{1}{\Gamma\left(\frac{a+b+1}{2}\right)} F\left(a, b ; \frac{a+b+1}{2} ; \frac{1-z}{2}\right)=\mathbf{F}_{\alpha, \alpha, 2 \lambda}\left(\frac{1-z}{2}\right), \\
\mathbf{S}_{\alpha, \lambda}^{\mathrm{I}}(z) & :=2^{-\frac{1}{2}-\alpha+\lambda} \frac{\Gamma\left(\frac{1+2 \alpha-2 \lambda}{2}\right) \Gamma\left(\frac{1+2 \lambda}{2}\right)}{\Gamma(\alpha+1)} S_{\alpha, \lambda}(z) \\
& =2^{-a} \frac{\Gamma(a) \Gamma\left(\frac{-a+b+1}{2}\right)}{\Gamma\left(\frac{a+b+1}{2}\right)} F\left(a, b ; \frac{a+b+1}{2} ; \frac{1-z}{2}\right) \\
& =2^{-\frac{1}{2}-\alpha+\lambda} \mathbf{F}_{\alpha, \alpha, 2 \lambda}^{\mathrm{I}}\left(\frac{1-z}{2}\right), \\
\mathbf{S}_{\alpha, \lambda}^{\mathrm{II}}(z) & :=\frac{\Gamma\left(\frac{1+2 \alpha-2 \lambda}{2}\right) \Gamma\left(\frac{1+2 \alpha+2 \lambda}{2}\right)}{\Gamma(2 \alpha+1)} S_{\alpha, \lambda}(z) \\
& =\frac{\Gamma(a) \Gamma(b)}{\Gamma(a+b)} F\left(a, b ; \frac{a+b+1}{2} ; \frac{1-z}{2}\right), \\
\mathbf{S}_{\alpha, \lambda}^{0}(z) & :=2^{2 \alpha} \frac{\Gamma\left(\frac{1+2 \alpha}{2}\right)^{2}}{\Gamma(2 \alpha+1)} S_{\alpha, \lambda}(z)=\sqrt{\pi} \frac{\Gamma\left(\frac{1+2 \alpha}{2}\right)}{\Gamma(\alpha+1)} S_{\alpha, \lambda}(z) \\
& =2^{a+b-1} \frac{\Gamma\left(\frac{a+b}{2}\right)^{2}}{\Gamma(a+b)} F\left(a, b ; \frac{a+b+1}{2} ; \frac{1-z}{2}\right) .
\end{aligned}
$$

Assuming that $z \notin]-\infty,-1$ ], we have the following integral representations: for $\operatorname{Re} \alpha+\frac{1}{2}>\operatorname{Re} \lambda>-\frac{1}{2}$

$$
\int_{-\infty}^{-1}\left(t^{2}-1\right)^{-\frac{1}{2}+\lambda}(z-t)^{-\frac{1}{2}-\alpha-\lambda} \mathrm{d} t=\mathbf{S}_{\alpha, \lambda}^{\mathrm{I}}(z),
$$


and for $\operatorname{Re} \alpha+\frac{1}{2}>|\operatorname{Re} \lambda|$

$$
\int_{0}^{\infty}\left(t^{2}+2 t z+1\right)^{-\alpha-\frac{1}{2}} t^{-\frac{1}{2}+\alpha+\lambda} \mathrm{d} t=\mathbf{S}_{\alpha, \lambda}^{\mathrm{II}}(z)
$$

6.10.2. Solution $\sim \mathbf{2}^{-\alpha}(\mathbf{1}-\boldsymbol{z})^{-\boldsymbol{\alpha}}$ at $\mathbf{1}$. If $\alpha \neq 1,2, \ldots$, then the unique solution of the Gegenbauer equation behaving as $(1-z)^{-\alpha}$ at 1 is the following function:

$$
\begin{aligned}
\left(1-z^{2}\right)^{-\alpha} S_{-\alpha,-\lambda}(z) & =2^{-\alpha}(1-z)^{-\alpha} F_{-\alpha, \alpha,-2 \lambda}\left(\frac{1-z}{2}\right) \\
& =\left(1-z^{2}\right)^{-\alpha} F_{-\alpha,-\frac{1}{2},-\lambda}\left(1-z^{2}\right) .
\end{aligned}
$$

Assuming that $z \notin]-\infty,-1] \cup[1, \infty[$, we have the following integral representations: for $-\operatorname{Re} \alpha+\frac{1}{2}>\operatorname{Re} \lambda>-\frac{1}{2}$

$$
\int_{-1}^{z}\left(1-t^{2}\right)^{-\frac{1}{2}+\lambda}(z-t)^{-\frac{1}{2}-\alpha-\lambda} \mathrm{d} t=\left(1-z^{2}\right)^{-\alpha} \mathbf{S}_{-\alpha, \lambda}^{\mathrm{I}}(z)
$$

and for $\frac{1}{2}>\operatorname{Re} \alpha$

$$
\int_{-\mathrm{i} \sqrt{1-z^{2}}-z}^{\mathrm{i} \sqrt{1-z^{2}}-z}\left(t^{2}+2 t z+1\right)^{-\alpha-\frac{1}{2}}(-t)^{-\frac{1}{2}+\alpha+\lambda} \mathrm{d} t=\left(1-z^{2}\right)^{-\alpha} \mathbf{S}_{\alpha, \lambda}^{0}(z) .
$$

6.10.3. Solution $\sim z^{-a}$ at $\infty$. If $2 \lambda \neq-1,-2, \ldots$, then the unique solution of the Gegenbauer equation behaving as $z^{-a}=z^{-\frac{1}{2}-\alpha+\lambda}$ at $\infty$ is the following function:

$$
\begin{aligned}
\left(z^{2}-1\right)^{\frac{-1-2 \alpha+2 \lambda}{4}} S_{-\lambda,-\alpha}\left(\frac{z}{\sqrt{z^{2}-1}}\right) & =(1+z)^{-\frac{1}{2}-\alpha+\lambda} F_{-2 \lambda, \alpha,-\alpha}\left(\frac{2}{1+z}\right) \\
& =z^{-\frac{1}{2}-\alpha+\lambda} F_{-\lambda, \alpha, \frac{1}{2}}\left(z^{-2}\right) .
\end{aligned}
$$

Assuming that $z \notin]-\infty, 1]$, we have the following integral representations: for $\frac{1}{2}>\operatorname{Re} \lambda$

$$
\int_{-1}^{1}\left(t^{2}-1\right)^{-\frac{1}{2}-\lambda}(z-t)^{-\frac{1}{2}-\alpha+\lambda} \mathrm{d} t=\left(z^{2}-1\right)^{\frac{-1-2 \alpha+2 \lambda}{4}} \mathbf{S}_{-\lambda, \alpha}^{0}\left(\frac{z}{\sqrt{z^{2}-1}}\right),
$$

and for $-\lambda+\frac{1}{2}>-\operatorname{Re} \alpha>-\frac{1}{2}$

$\int_{\sqrt{z^{2}-1}-z}^{0}\left(t^{2}+2 t z+1\right)^{-\alpha-\frac{1}{2}}(-t)^{-\frac{1}{2}+\alpha-\lambda} \mathrm{d} t=\left(z^{2}-1\right)^{\frac{-1-2 \alpha+2 \lambda}{4}} \mathbf{S}_{-\lambda, \alpha}^{\mathrm{I}}\left(\frac{z}{\sqrt{z^{2}-1}}\right)$. 
6.10.4. Solution $\sim \boldsymbol{z}^{-\boldsymbol{b}}$ at $\boldsymbol{\infty}$. If $2 \lambda \neq 1,2, \ldots$, then the unique solution of the Gegenbauer equation behaving as $z^{-b}=z^{-\frac{1}{2}-\alpha-\lambda}$ at $\infty$ is the following function:

$$
\begin{aligned}
\left(z^{2}-1\right)^{\frac{-1-2 \alpha-2 \lambda}{4}} S_{\lambda, \alpha}\left(\frac{z}{\sqrt{z^{2}-1}}\right) & =(1+z)^{-\frac{1}{2}-\alpha-\lambda} F_{2 \lambda, \alpha, \alpha}\left(\frac{2}{1+z}\right) \\
& =z^{-\frac{1}{2}-\alpha-\lambda} F_{\lambda, \alpha, \frac{1}{2}}\left(z^{-2}\right) .
\end{aligned}
$$

Assuming that $z \notin]-\infty, 1]$, we have the following integral representations: for $\operatorname{Re} \lambda+\frac{1}{2}>|\operatorname{Re} \alpha|$

$$
\int_{z}^{\infty}\left(t^{2}-1\right)^{-\frac{1}{2}-\lambda}(t-z)^{-\frac{1}{2}-\alpha+\lambda} \mathrm{d} t=\left(z^{2}-1\right)^{\frac{-1-2 \alpha-2 \lambda}{4}} \mathbf{S}_{\lambda, \alpha}^{\mathrm{II}}\left(\frac{z}{\sqrt{z^{2}-1}}\right),
$$

and for $\lambda+\frac{1}{2}>-\operatorname{Re} \alpha>-\frac{1}{2}$

$$
\int_{\infty}^{-\sqrt{z^{2}-1}-z}\left(t^{2}+2 t z+1\right)^{-\alpha-\frac{1}{2}} t^{-\frac{1}{2}+\alpha-\lambda} \mathrm{d} t=\left(z^{2}-1\right)^{-\frac{1}{4}-\frac{\alpha}{2}-\frac{\lambda}{2}} \mathbf{S}_{\lambda, \alpha}^{\mathrm{I}}\left(\frac{z}{\sqrt{z^{2}-1}}\right) .
$$

Remark 6.3. As mentioned in Remark 6.2, in the literature instead of the Gegenbauer equation the associated Legendre equation usually appears. One class of its standard solutions is the associated Legendre function of the 1st kind

$$
\begin{aligned}
\mathbf{P}_{l}^{m}(z) & =\left(\frac{z+1}{z-1}\right)^{\frac{m}{2}} \mathbf{F}\left(-l, l+1 ; 1-m ; \frac{1-z}{2}\right) \\
& =\frac{2^{m}}{\left(z^{2}-1\right)^{\frac{m}{2}}} \mathbf{F}\left(1-m+l,-m-l ; 1-m ; \frac{1-z}{2}\right) \\
& =\frac{2^{m}}{\left(z^{2}-1\right)^{\frac{m}{2}}} \mathbf{S}_{-m, l+\frac{1}{2}}(z),
\end{aligned}
$$

which up to a constant are $\left(z^{2}-1\right)^{\frac{m}{2}}$ times the solutions of Sect. 6.10.2. Another class of solutions is the associated Legendre function of the 2nd kind

$$
\begin{aligned}
\mathbf{Q}_{l}^{m}(z) & =\frac{\left(z^{2}-1\right)^{\frac{m}{2}}}{2^{l+1} z^{l+m+1}} \mathbf{F}\left(\frac{l+m+2}{2}, \frac{l+m+1}{2} ; l+\frac{3}{2} ; z^{-2}\right) \\
& =2^{-l-1}\left(z^{2}-1\right)^{-\frac{l+1}{2}} \mathbf{S}_{l+\frac{1}{2}, m}\left(\frac{z}{\sqrt{z^{2}-1}}\right),
\end{aligned}
$$

which up to a constant are $\left(z^{2}-1\right)^{\frac{m}{2}}$ times the solutions of Sect. 6.10.4. (In the literature, one can find a couple of other varieties of associated Legendre functions of the 1 st and 2 nd kind, differing by their normalization, see e.g. [12]).

\subsection{Connection Formulas}

We can express the standard solutions in terms of the even and odd solutions

$$
\begin{aligned}
\mathbf{S}_{\alpha, \lambda}(z)= & \frac{\sqrt{\pi}}{\Gamma\left(\frac{3}{4}+\frac{\alpha}{2}-\frac{\lambda}{2}\right) \Gamma\left(\frac{3}{4}+\frac{\alpha}{2}+\frac{\lambda}{2}\right)} S_{\alpha, \lambda}^{+}(z) \\
& +\frac{\sqrt{\pi}}{\Gamma\left(\frac{1}{4}+\frac{\alpha}{2}-\frac{\lambda}{2}\right) \Gamma\left(\frac{1}{2}+\frac{\alpha}{2}+\frac{\lambda}{2}\right)} S_{\alpha, \lambda}^{-}(z),
\end{aligned}
$$




$$
\begin{aligned}
\left(1-z^{2}\right)^{-\alpha} \mathbf{S}_{-\alpha,-\lambda}(z)= & \frac{\sqrt{\pi}}{\Gamma\left(\frac{3}{4}-\frac{\alpha}{2}+\frac{\lambda}{2}\right) \Gamma\left(\frac{3}{4}-\frac{\alpha}{2}-\frac{\lambda}{2}\right)} S_{\alpha, \lambda}^{+}(z) \\
& +\frac{\sqrt{\pi}}{\Gamma\left(\frac{1}{4}-\frac{\alpha}{2}+\frac{\lambda}{2}\right) \Gamma\left(\frac{1}{4}-\frac{\alpha}{2}-\frac{\lambda}{2}\right)} S_{\alpha, \lambda}^{-}(z), \\
\left(1-z^{2}\right)^{-\frac{1}{4}-\frac{\alpha}{2}+\frac{\lambda}{2}} \mathbf{S}_{-\lambda,-\alpha}\left(\frac{z}{\sqrt{z^{2}-1}}\right)= & \frac{\sqrt{\pi}}{\Gamma\left(\frac{3}{4}-\frac{\alpha}{2}-\frac{\lambda}{2}\right) \Gamma\left(\frac{3}{4}+\frac{\alpha}{2}-\frac{\lambda}{2}\right)} S_{\alpha, \lambda}^{+}(z) \\
& +\frac{\mathrm{i} \sqrt{\pi}}{\Gamma\left(\frac{1}{4}-\frac{\alpha}{2}-\frac{\lambda}{2}\right) \Gamma\left(\frac{1}{4}+\frac{\alpha}{2}-\frac{\lambda}{2}\right)} S_{\alpha, \lambda}^{-}(z), \\
\left(1-z^{2}\right)^{-\frac{1}{4}-\frac{\alpha}{2}-\frac{\lambda}{2}} \mathbf{S}_{\lambda, \alpha}\left(\frac{z}{\sqrt{z^{2}-1}}\right)= & \frac{\sqrt{\pi}}{\Gamma\left(\frac{3}{4}-\frac{\alpha}{2}+\frac{\lambda}{2}\right) \Gamma\left(\frac{3}{4}+\frac{\alpha}{2}+\frac{\lambda}{2}\right)} S_{\alpha, \lambda}^{+}(z) \\
& +\frac{\mathrm{i} \sqrt{\pi}}{\Gamma\left(\frac{1}{4}-\frac{\alpha}{2}+\frac{\lambda}{2}\right) \Gamma\left(\frac{1}{4}+\frac{\alpha}{2}+\frac{\lambda}{2}\right)} S_{\alpha, \lambda}^{-}(z) .
\end{aligned}
$$

\subsection{Recurrence Relations}

The following recurrence relations can be easily derived from the commutation properties of Sect. 6.4

$$
\begin{gathered}
\partial_{z} \mathbf{S}_{\alpha, \lambda}(z)=-\frac{1}{2}\left(\frac{1}{2}+\alpha-\lambda\right)\left(\frac{1}{2}+\alpha+\lambda\right) \mathbf{S}_{\alpha+1, \lambda}(z) \\
\left(\left(1-z^{2}\right) \partial_{z}-2 \alpha z\right) \mathbf{S}_{\alpha, \lambda}(z)=-2 \mathbf{S}_{\alpha-1, \lambda}(z) \\
\left(\left(1-z^{2}\right) \partial_{z}-\left(\frac{1}{2}+\alpha+\lambda\right) z\right) \mathbf{S}_{\alpha, \lambda}(z)=-\left(\frac{1}{2}+\alpha+\lambda\right) \mathbf{S}_{\alpha, \lambda+1}(z) \\
\left(\left(1-z^{2}\right) \partial_{z}-\left(\frac{1}{2}+\alpha-\lambda\right) z\right) \mathbf{S}_{\alpha, \lambda}(z)=-\left(\frac{1}{2}+\alpha-\lambda\right) \mathbf{S}_{\alpha, \lambda-1}(z) \\
\left(z \partial_{z}+\frac{1}{2}+\alpha-\lambda\right) \mathbf{S}_{\alpha, \lambda}(z)=\frac{1}{2}\left(\frac{1}{2}+\alpha-\lambda\right)\left(\frac{3}{2}+\alpha-\lambda\right) \mathbf{S}_{\alpha+1, \lambda-1}(z) \\
\left(z\left(1-z^{2}\right) \partial_{z}+\left(\frac{1}{2}-\alpha+\lambda\right)\left(1-z^{2}\right)-2 \alpha z^{2}\right) \mathbf{S}_{\alpha, \lambda}(z)=-2 \mathbf{S}_{\alpha-1, \lambda+1}(z) \\
\left(z \partial_{z}+\frac{1}{2}+\alpha+\lambda\right) \mathbf{S}_{\alpha, \lambda}(z)=\left(\frac{1}{2}+\alpha+\lambda\right)(\alpha+1) \mathbf{S}_{\alpha+1, \lambda+1}(z) \\
\left(z\left(1-z^{2}\right) \partial_{z}+\left(\frac{1}{2}-\alpha-\lambda\right)\left(1-z^{2}\right)-2 \alpha z^{2}\right) \mathbf{S}_{\alpha, \lambda}(z)=-2 \mathbf{S}_{\alpha-1, \lambda-1}(z)
\end{gathered}
$$

\subsection{Gegenbauer Polynomials}

If $-a=n=0,1,2, \ldots$, then Gegenbauer functions are polynomials.

We will use two distinct normalizations of these polynomials. The $C_{n}^{\text {I }}$ polynomials have a natural Rodriguez-type definition:

$$
C_{n}^{\mathrm{I}, \alpha}(z):=\frac{1}{2^{n} n !}\left(z^{2}-1\right)^{-\alpha} \partial_{z}^{n}\left(z^{2}-1\right)^{n+\alpha} .
$$

The $C_{n}^{\text {II }}$ polynomials are defined as

$$
C_{n}^{\mathrm{II}, \alpha}(z):=\frac{(2 \alpha+1)_{n}}{(\alpha+1)_{n}} C_{n}^{\mathrm{I}, \alpha}(z) .
$$


Remark 6.4. The first kind polynomials is just the special case of the conventional Jacobi polynomials (see Remark 3.3) with $\alpha=\beta$ :

$$
C_{n}^{\mathrm{I}, \alpha}(z)=P_{n}^{\alpha, \alpha}(z)
$$

The second kind of polynomials is called in the literature the Gegenbauer polynomials. In the standard notation, its parameter is shifted by $\frac{1}{2}$ :

$$
C_{n}^{\mathrm{II}, \alpha}(z)=C_{n}^{\alpha+\frac{1}{2}}(z) .
$$

When describing the properties of Gegenbauer polynomials, we can choose between $C_{n}^{\mathrm{I}}$ and $C_{n}^{\text {II }}$. We either give properties of both kinds of polynomials or choose those that give simpler formulas.

Both kinds of polynomials solve the Gegenbauer equation:

$$
\begin{aligned}
& \left(\left(1-z^{2}\right) \partial_{z}^{2}-2(1+\alpha) z \partial_{z}+n(n+2 \alpha+1)\right) C_{n}^{\mathrm{I} / \mathrm{II}}(z) \\
& \quad=\mathcal{S}\left(-n, n+2 \alpha+1 ; z, \partial_{z}\right) C_{n}^{\mathrm{I} / \mathrm{II}}(z)=0 .
\end{aligned}
$$

Generating functions:

$$
\begin{aligned}
\left(1-2 t z+t^{2}\left(z^{2}-1\right)\right)^{-\alpha} & =\sum_{n=0}^{\infty}(2 t)^{n} C_{n}^{\mathrm{I},-\alpha-n}(z), \\
\left(1-2 z t+t^{2}\right)^{-\alpha-\frac{1}{2}} & =\sum_{n=0}^{\infty} C_{n}^{\mathrm{II}, \alpha}(z) t^{n} .
\end{aligned}
$$

Integral representations:

$$
\begin{aligned}
C_{n}^{\mathrm{I}, \alpha}(z) & =\frac{1}{2 \pi \mathrm{i}} \int_{\left[0^{+}\right]}\left(1-2 t z+t^{2}\left(z^{2}-1\right)\right)^{\alpha+n} t^{-n-1} \mathrm{~d} t \\
C_{n}^{\mathrm{II}, \alpha}(z) & =\frac{1}{2 \pi \mathrm{i}} \int_{\left[0^{+}\right]}\left(1-2 z t+t^{2}\right)^{-\alpha-\frac{1}{2}} t^{-n-1} \mathrm{~d} t .
\end{aligned}
$$

We give symmetries for both kinds of polynomials:

$$
\begin{aligned}
C_{n}^{\mathrm{I}, \alpha}(z) & =(-1)^{n} C_{n}^{\mathrm{I}, \alpha}(-z) \\
& =\frac{(2 \alpha+1+n)_{n}}{(\mp 2)^{n}\left(\alpha+\frac{1}{2}\right)_{n}}\left(z^{2}-1\right)^{\frac{n}{2}} C_{n}^{\mathrm{I},-\frac{1}{2}-\alpha-n}\left(\frac{ \pm z}{\sqrt{z^{2}-1}}\right) . \\
C_{n}^{\mathrm{II}, \alpha}(z) & =(-1)^{n} C_{n}^{\mathrm{II}, \alpha}(-z) \\
& =\frac{(\mp 2)^{n}\left(\alpha+\frac{1}{2}\right)_{n}}{(2 \alpha+1+n)_{n}}\left(z^{2}-1\right)^{\frac{n}{2}} C_{n}^{\mathrm{II},-\frac{1}{2}-\alpha-n}\left(\frac{ \pm z}{\sqrt{z^{2}-1}}\right) .
\end{aligned}
$$

We give recurrence relations only for $C_{n}^{\mathrm{II}, \alpha}$, those for $C_{n}^{\mathrm{I}, \alpha}$ differ by coefficients on the right, but have a comparable level of complexity:

$$
\begin{gathered}
\partial_{z} C_{n}^{\mathrm{II}, \alpha}(z)=(2 \alpha+1) C_{n-1}^{\mathrm{II}, \alpha+1}(z) \\
\left(\left(1-z^{2}\right) \partial_{z}-2 \alpha z\right) C_{n}^{\mathrm{II}, \alpha}(z)=\frac{-(n+1)(n+2 \alpha)}{2 \alpha} C_{n+1}^{\mathrm{II}, \alpha-1}(z),
\end{gathered}
$$




$$
\begin{gathered}
\left(\left(1-z^{2}\right) \partial_{z}-(n+2 \alpha+1) z\right) C_{n}^{\mathrm{II}, \alpha}(z)=-(n+1) C_{n+1}^{\mathrm{II}, \alpha}(z), \\
\left(\left(1-z^{2}\right) \partial_{z}+n z\right) C_{n}^{\mathrm{II}, \alpha}(z)=(n+2 \alpha) C_{n-1}^{\mathrm{II}, \alpha}(z), \\
\left(z \partial_{z}-n\right) C_{n}^{\mathrm{II}, \alpha}(z)=(2 \alpha+1) C_{n-2}^{\mathrm{II}, \alpha+1}(z), \\
\left(z\left(1-z^{2}\right) \partial_{z}+1+n-(n+2 \alpha+1) z^{2}\right) C_{n}^{\mathrm{II}, \alpha}(z)=-\frac{(n+1)(n+2)}{2 \alpha-1} C_{n+2}^{\mathrm{II}, \alpha-1}(z), \\
\left(z \partial_{z}+n+2 \alpha+1\right) C_{n}^{\mathrm{II}, \alpha}(z)=(2 \alpha+1) C_{n}^{\mathrm{II}, \alpha+1}(z), \\
\left(z\left(1-z^{2}\right) \partial_{z}-n-2 \alpha+n z^{2}\right) C_{n}^{\mathrm{II}, \alpha}(z)=-\frac{(2 \alpha+n-1)(2 \alpha+n)}{2 \alpha-1} C_{n}^{\mathrm{II}, \alpha-1}(z) .
\end{gathered}
$$

The differential equation, the Rodriguez-type formula, the first generating function and the first integral representation are special cases of the corresponding formulas of Sect. 1.6. Thus, the polynomials $C^{\mathrm{I}}$ belong to the scheme of Sect. 1.6. $C^{\text {II }}$ do not have a natural Rodriguez-type formula, and do not belong to the scheme of Sect. 1.6.

The $C^{\mathrm{I}}$ polynomials have simple expressions in terms of the Jacobi polynomials:

$$
\begin{aligned}
C_{n}^{\mathrm{I}, \alpha}(z) & =( \pm 1)^{n} R_{n}^{\alpha, \alpha}\left(\frac{1 \mp z}{2}\right) \\
& =\left(\frac{ \pm 1-z}{2}\right)^{n} R_{n}^{\alpha,-2 \alpha-2 n-1}\left(\frac{2}{1 \mp z}\right) \\
& =\left(\frac{z \mp 1}{2}\right)^{n} R_{n}^{-2 \alpha-2 n-1, \alpha}\left(\frac{ \pm 1+z}{\mp 1+z}\right) .
\end{aligned}
$$

We have several alternative expressions for $C^{\mathrm{I}}$ and $C^{\mathrm{II}}$ polynomials:

$$
\begin{aligned}
C_{n}^{\mathrm{I}, \alpha}(z) & :=\lim _{\nu \rightarrow n}(-1)^{n}(\nu-n) \mathbf{S}_{\alpha, \nu+\alpha+\frac{1}{2}}^{\mathrm{I}}(z)=\lim _{\nu \rightarrow n}(\nu-n) \mathbf{F}_{\alpha, \alpha, 2 \nu+2 \alpha+1}^{\mathrm{I}}\left(\frac{1 \mp z}{2}\right) \\
& =( \pm 1)^{n} \frac{(\alpha+1)_{n}}{n !} F\left(-n, n+2 \alpha+1 ; \alpha+1 ; \frac{1 \mp z}{2}\right), \\
C_{n}^{\mathrm{II}, \alpha}(z) & :=\lim _{\nu \rightarrow n}(-1)^{n}(\nu-n) \mathbf{S}_{\alpha, \nu+\alpha+\frac{1}{2}}^{\mathrm{II}}(z) \\
& =( \pm 1)^{n} \frac{(2 \alpha+1)_{n}}{n !} F\left(-n, n+2 \alpha+1 ; \alpha+1 ; \frac{1 \mp z}{2}\right) \\
& =\sum_{k=0}^{\left[\frac{n}{2}\right]} \frac{(-1)^{k}\left(\alpha+\frac{1}{2}\right)_{n-k}}{k !(n-2 k) !}(2 z)^{n-2 k} .
\end{aligned}
$$

Values at \pm 1 , for behavior at infinity, we give for both kinds of polynomials:

$$
\begin{aligned}
C_{n}^{\mathrm{I}, \alpha}( \pm 1) & =( \pm 1)^{n} \frac{(\alpha+1)_{n}}{n !}, & \lim _{z \rightarrow \infty} \frac{C_{n}^{\mathrm{I}, \alpha}(z)}{z^{n}}=\frac{2^{-n}(2 \alpha+n+1)_{n}}{n !}, \\
C_{n}^{\mathrm{II}, \alpha}( \pm 1) & =( \pm 1)^{n} \frac{(2 \alpha+1)_{n}}{n !}, & \lim _{z \rightarrow \infty} \frac{C_{n}^{\mathrm{II}, \alpha}(z)}{z^{n}}=\frac{2^{n}\left(\alpha+\frac{1}{2}\right)_{n}}{n !} .
\end{aligned}
$$


The degenerate case has a simple expression in terms of $C^{\mathrm{I}}$ polynomials:

$$
C_{n}^{\mathrm{I}, \alpha}=\left(\frac{2}{z^{2}-1}\right)^{\alpha} C_{n+2 \alpha}^{\mathrm{I},-\alpha}(z), \quad \alpha \in \mathbb{Z} .
$$

The initial conditions at 0 and the identities for the even and odd case are given only for $C_{n}^{\mathrm{II}, \alpha}$, since those for $C_{n}^{\mathrm{I}, \alpha}$ are more complicated:

$$
\begin{aligned}
C_{2 m}^{\mathrm{II}, \alpha}(0)= & \frac{(-1)^{m}\left(\alpha+\frac{1}{2}\right)_{m}}{m !}, \quad \partial_{z} C_{2 m}^{\mathrm{II}, \alpha}(0)=0 ; \\
C_{2 m+1}^{\mathrm{II}, \alpha}(0)= & , \quad \partial_{z} C_{2 m+1}^{\mathrm{II}, \alpha}(0)=\frac{(-1)^{m} 2\left(\alpha+\frac{1}{2}\right)_{m}}{m !} . \\
C_{2 m}^{\mathrm{II}, \alpha}(z) & =(-1)^{m} \frac{\left(\alpha+\frac{1}{2}\right)_{m}}{(\alpha+1)_{m}} R_{m}^{\alpha,-\frac{1}{2}}\left(z^{2}\right) \\
& =(-1)^{m} \frac{\left(\alpha+\frac{1}{2}\right)_{m}}{m !} S_{\alpha, 2 m+\frac{1}{2}+\alpha}^{+}(z) \\
& =(-1)^{m} \frac{\left(\alpha+\frac{1}{2}\right)_{m}}{m !} F\left(-m, m+\frac{1}{2}+\alpha ; \frac{1}{2} ; z^{2}\right) \\
C_{2 m+1}^{\mathrm{II}, \alpha}(z) & =(-1)^{m} \frac{\left(\alpha+\frac{1}{2}\right)_{m}}{(\alpha+1)_{m}} 2 z R_{m}^{\alpha, \frac{1}{2}}\left(z^{2}\right) \\
& =(-1)^{m} \frac{\left(\alpha+\frac{1}{2}\right)_{m}}{m !} S_{\alpha, 2 m+\frac{3}{2}+\alpha}^{-}(z) \\
& =(-1)^{m} \frac{\left(\alpha+\frac{1}{2}\right)_{m}}{m !} 2 z F\left(-m, m+\frac{3}{2}+\alpha ; \frac{3}{2} ; z^{2}\right) .
\end{aligned}
$$

We have the following special cases:

1. If $\alpha \in \mathbb{Z},-n \leq \alpha \leq \frac{-n-1}{2}$, then $C_{n}^{\mathrm{I}, \alpha}=0$.

2. If $\alpha \in \mathbb{Z}+\frac{1}{2}, \frac{-n-1}{2} \leq \alpha \leq-\frac{1}{2}$, then $C_{n}^{\mathrm{II}, \alpha}=0$.

3. If $\alpha \in \mathbb{Z}, \frac{-n+1}{2} \leq \alpha \leq-1$, then $C_{n}^{\mathrm{I} / \mathrm{II}, \alpha}=\left(1-z^{2}\right)^{-\alpha} W$, where $W$ is a polynomial not divisible by $1-z^{2}$.

\subsection{Special Cases}

When describing special cases of the Gegenbauer equation, we will primarily use the Lie-algebraic parameters.

6.14.1. The Legendre Equation. Suppose that one of the parameters is an integer. Using, if necessary, recurrence relations, we can assume that it is zero. After applying an appropriate symmetry, we can assume that $\alpha=0$. We obtain then the Legendre operator:

$$
\mathcal{S}_{0, \lambda}\left(z, \partial_{z}\right)=\left(1-z^{2}\right) \partial_{z}^{2}-2 z \partial_{z}+\lambda^{2}-\frac{1}{4} .
$$

For the particular case $\lambda=0$, its solutions can be expressed by the so-called complete elliptic functions.

The Legendre operator for polynomials of degree $n$ has the form

$$
\left(1-z^{2}\right) \partial_{z}^{2}-2 z \partial_{z}+n(n+1) .
$$


The Legendre polynomials are special cases of both $C^{\mathrm{I}}$ and $C^{\mathrm{II}}$ :

$$
\begin{aligned}
P_{n}(z) & =C_{n}^{\mathrm{I}, 0}(z)=C_{n}^{\mathrm{II}, 0}(z) \\
& =\frac{1}{2^{n} n !} \partial_{z}^{n}\left(z^{2}-1\right)^{n} .
\end{aligned}
$$

Their generating function is a special case of the generating function for $C^{\mathrm{II}}$ :

$$
\left(1-2 z t+t^{2}\right)^{-\frac{1}{2}}=\sum_{n=0}^{\infty} P_{n}(z) t^{n} .
$$

6.14.2. Chebyshev Equation of the 1st Kind. Suppose that one of the parameters belongs to $\mathbb{Z}+\frac{1}{2}$. Using, if necessary, recurrence relation, we can assume that it equals $-\frac{1}{2}$. After applying an appropriate symmetry, we can assume that $\alpha=-\frac{1}{2}$. We obtain then the Chebyshev operator of the 1 st kind:

$$
\mathcal{S}_{0, \lambda}\left(z, \partial_{z}\right)=\left(1-z^{2}\right) \partial_{z}^{2}-2 z \partial_{z}+\lambda^{2} .
$$

After substitution $z=\cos \phi$, it becomes

$$
\partial_{\phi}^{2}+\lambda^{2}
$$

Thus, the corresponding equation can be solved in terms of elementary functions.

To obtain an operator that annihilates a polynomial of degree $n$, we simply set $\lambda=n$ :

$$
\left(1-z^{2}\right) \partial_{z}^{2}-2 z \partial_{z}+n^{2}
$$

The Chebyshev polynomials of the 1st kind are

$$
\begin{aligned}
T_{n}(z) & =\frac{n !}{(1 / 2)_{n}} C_{n}^{\mathrm{I},-\frac{1}{2}}(z)=\frac{\mathrm{d}}{\mathrm{d} \alpha} C_{n}^{\mathrm{II},-\frac{1}{2}}(z) \\
& =\frac{1}{2}\left(\left(z+\mathrm{i} \sqrt{1-z^{2}}\right)^{n}+\left(z-\mathrm{i} \sqrt{1-z^{2}}\right)^{n}\right) .
\end{aligned}
$$

Note that $C_{n}^{\mathrm{II},-\frac{1}{2}}=0$, therefore, the usual generating function for $C^{\mathrm{II}}$ cannot be applied for the Chebyshev polynomials of the 1st kind. Instead, we have generating functions

$$
\begin{aligned}
-\log \left(1-2 z t+t^{2}\right) & =\sum_{n=0}^{\infty} T_{n}(z) \frac{t^{n}}{n}, \\
\frac{1-z t}{1-2 z t+t^{2}} & =\sum_{n=0}^{\infty} T_{n}(z) t^{n} .
\end{aligned}
$$

6.14.3. Chebyshev Equation of the 2nd Kind. If one of the parameters belongs to $\mathbb{Z}+\frac{1}{2}$, instead of $\alpha=-\frac{1}{2}$ we can reduce ourselves to the case $\alpha=\frac{1}{2}$. We obtain then the Chebyshev operator of the 2nd kind:

$$
\mathcal{S}_{0, \lambda}\left(z, \partial_{z}\right)=\left(1-z^{2}\right) \partial_{z}^{2}-2 z \partial_{z}+\lambda^{2}-1 .
$$

After substitution $z=\cos \phi$ it becomes

$$
\sin \phi\left(\partial_{\phi}^{2}+\lambda^{2}\right)(\sin \phi)^{-1}
$$


Clearly, the corresponding equation can also be solved in elementary functions.

To obtain an operator that annihilates a polynomial of degree $n$, we set $\lambda=n+1$ :

$$
\left(1-z^{2}\right) \partial_{z}^{2}-2 z \partial_{z}+n(n+2)
$$

The Chebyshev polynomials of the 2nd kind are

$$
\begin{aligned}
U_{n}(z) & =\frac{n !}{(3 / 2)_{n}} C_{n}^{\mathrm{I}, \frac{1}{2}}(z)=C_{n}^{\mathrm{II}, \frac{1}{2}}(z) \\
& =\frac{\left(z+\mathrm{i} \sqrt{1-z^{2}}\right)^{n+1}-\left(z-\mathrm{i} \sqrt{1-z^{2}}\right)^{n+1}}{2 \mathrm{i} \sqrt{1-z^{2}}} .
\end{aligned}
$$

Their generating function is a special case of the generating function for $C^{\mathrm{II}}$ :

$$
\left(1-2 z t+t^{2}\right)^{-1}=\sum_{n=0}^{\infty} U_{n}(z) t^{n} .
$$

\section{The Hermite Equation}

\subsection{Introduction}

Let $a \in \mathbb{C}$. In this section, we study the Hermite equation, which is given by the operator

$$
\mathcal{S}\left(a, z, \partial_{z}\right):=\partial_{z}^{2}-2 z \partial_{z}-2 a
$$

The choice of the parameter $a$ is dictated by the analogy with the parameters of the Gegenbauer. It will be called a classical parameter, even though it is not the usual one in the literature.

The Hermite operator can be obtained as the limit of the Gegenbauer operator:

$$
\lim _{b \rightarrow \infty} \frac{2}{b} \mathcal{S}\left(a, b ; z \sqrt{2 / b}, \partial_{(z \sqrt{2 / b})}\right)=\mathcal{S}(a ; z, \partial z) .
$$

To describe the symmetries it is convenient to use its Lie-algebraic parameter:

$$
\lambda=a-\frac{1}{2}, \quad a=\lambda+\frac{1}{2} .
$$

In the new parameter the Hermite operator equals

$$
\mathcal{S}_{\lambda}\left(z, \partial_{z}\right)=\partial_{z}^{2}-2 z \partial_{z}-2 \lambda-1
$$

The Lie-algebraic parameter has an interesting interpretation in terms of a "Cartan element" of the Lie algebra sch(1) [2]. 


\subsection{Equivalence with a Subclass of the Confluent Equation}

The Hermite equation is reflection invariant. Using the quadratic transformation, we can reduce it to a special case of the confluent equation:

$$
\begin{aligned}
\mathcal{S}\left(a ; z, \partial_{z}\right) & =4 \mathcal{F}\left(\frac{a}{2} ; \frac{1}{2} ; w, \partial_{w}\right), \\
z^{-1} \mathcal{S}\left(a ; z, \partial_{z}\right) z & =4 \mathcal{F}\left(\frac{a+1}{2} ; \frac{3}{2} ; w, \partial_{w}\right),
\end{aligned}
$$

where

$$
w=z^{2}, \quad z=\sqrt{w} .
$$

In the Lie-algebraic parameters

$$
\begin{aligned}
\mathcal{S}_{\lambda}\left(z, \partial_{z}\right) & =4 \mathcal{F}_{\lambda,-\frac{1}{2}}\left(w, \partial_{w}\right) \\
z^{-1} \mathcal{S}_{\lambda}\left(z, \partial_{z}\right) z & =4 \mathcal{F}_{\lambda, \frac{1}{2}}\left(w, \partial_{w}\right) .
\end{aligned}
$$

\subsection{Symmetries}

The following operators equal $\mathcal{S}_{\lambda}\left(w, \partial_{w}\right)$ for an appropriate $w$ :

$$
\begin{aligned}
& w= \pm z: \\
& w= \pm \mathrm{i} z: \quad \mathcal{S}_{\lambda}\left(z, \partial_{z}\right), \\
& -\exp \left(-z^{2}\right) \quad \mathcal{S}_{-\lambda}\left(z, \partial_{z}\right) \quad \exp \left(z^{2}\right) .
\end{aligned}
$$

The group of symmetries of the Hermite equation is isomorphic to $\mathbb{Z}_{4}$ and can be interpreted as the "Weyl group" of $s c h(1)$.

\subsection{Factorizations and Commutation Properties}

There are several ways to factorize the Hermite operator:

$$
\begin{aligned}
\mathcal{S}_{\lambda}= & \left(\partial_{z}-2 z\right) \partial_{z}-2 \lambda-1 \\
= & \partial_{z}\left(\partial_{z}-2 z\right)-2 \lambda+1, \\
z^{2} \mathcal{S}_{\lambda}= & \left(z \partial_{z}+\lambda-\frac{3}{2}\right)\left(z \partial_{z}-\lambda+\frac{1}{2}-2 z^{2}\right) \\
& +\left(\lambda-\frac{3}{2}\right)\left(\lambda-\frac{1}{2}\right) \\
= & \left(z \partial_{z}-\lambda-\frac{3}{2}-2 z^{2}\right)\left(z \partial_{z}+\lambda+\frac{1}{2}\right) \\
& +\left(\lambda+\frac{3}{2}\right)\left(\lambda+\frac{1}{2}\right) .
\end{aligned}
$$


The factorizations can be used to derive the following commutation relations:

$$
\begin{aligned}
\partial_{z} & \mathcal{S}_{\lambda} \\
=\mathcal{S}_{\lambda+1} & \partial_{z} \\
\left(\partial_{z}-2 z\right) & \mathcal{S}_{\lambda} \\
=\mathcal{S}_{\lambda-1} & \left(\partial_{z}-2 z\right) \\
\left(z \partial_{z}+\lambda+\frac{1}{2}\right) & z^{2} \mathcal{S}_{\lambda} \\
=z^{2} \mathcal{S}_{\lambda+2} & \left(z \partial_{z}+\lambda+\frac{1}{2}\right) \\
\left(z \partial_{z}-\lambda+\frac{1}{2}-2 z^{2}\right) & z^{2} \mathcal{S}_{\lambda} \\
=z^{2} \mathcal{S}_{\lambda-2} & \left(z \partial_{z}-\lambda+\frac{1}{2}-2 z^{2}\right) .
\end{aligned}
$$

Each of these commutations relations is associated with a "root" of the Lie algebra $\operatorname{sch}(1)$.

\subsection{Convergence of the Gegenbauer Equation to the Hermite Equation}

It is interesting to describe the transition from the symmetries of the Gegenbauer equation to the symmetries of the Hermite equation. We consider the limit (7.1). We also consider the surface $\Omega$ described in Sect. 6.5.

Let us look only at the part of $\Omega$ given by the union of $\Omega_{+} \cap\{\operatorname{Im} z>0\}_{+}$ and $\Omega_{-} \cap\{\operatorname{Im} z>0\}_{-}$glued along $]-1,1[$. The scaling involved in the limit (7.1) transforms this part of $\Omega$ into $\mathbb{C}$.

$\tau\left(\Omega_{+} \cap\{\operatorname{Im} z>0\}\right)$ is equal to the union of $\Omega_{-} \cap\{\operatorname{Im} z>0, \operatorname{Re} z>0\}$ and $\Omega_{-} \cap\{\operatorname{Im} z<0, \operatorname{Re} z>0\}$ glued along ]0,1[. Thus, the limit of $\tau$ on $\Omega_{+} \cap\{\operatorname{Im} z>0\}$ equals the multiplication by $-\mathrm{i}$.

$-\tau\left(\Omega_{-} \cap\{\operatorname{Im} z<0\}\right)$ is equal to the union of $\Omega_{+} \cap\{\operatorname{Im} z>0, \operatorname{Re} z<0\}$ and $\Omega_{-} \cap\{\operatorname{Im} z<0, \operatorname{Re} z<0\}$ glued along $]-1,0[$. Thus, the limit of $-\tau$ on $\Omega_{-} \cap\{\operatorname{Im} z>0\}$ also equals the multiplication by $-\mathrm{i}$.

Thus, the multiplication by $-\mathrm{i}$ is not the limit of a single element of the group of the symmetries of the Gegenbauer equation, but a combination of the limits of two symmetries.

\subsection{Integral Representations}

Below, we describe two kinds of integral representations of the Hermite equation.

Theorem 7.1. 1. Let $[0,1] \ni t \mapsto \gamma(t)$ satisfy

$$
\left.\mathrm{e}^{t^{2}}(t-z)^{-a-1}\right|_{\gamma(0)} ^{\gamma(1)}=0 \text {. }
$$

Then

$$
\mathcal{S}\left(a ; z, \partial_{z}\right) \int_{\gamma} \mathrm{e}^{t^{2}}(t-z)^{-a} \mathrm{~d} t .
$$

2. Let $[0,1] \ni t \mapsto \gamma(t)$ satisfy

$$
\left.\mathrm{e}^{-t^{2}-2 z t} t^{a}\right|_{\gamma(0)} ^{\gamma(1)}=0 .
$$


Then

$$
\mathcal{S}\left(a ; z, \partial_{z}\right) \int_{\gamma} \mathrm{e}^{-t^{2}-2 z t} t^{a-1} \mathrm{~d} t=0 .
$$

Proof. We check that for any contour $\gamma,(7.4)$ and (7.5) equal

$$
\begin{aligned}
& -a \int_{\gamma}\left(\partial_{t} \mathrm{e}^{t^{2}}(t-z)^{-a-1}\right) \mathrm{d} t \\
& -2 \int_{\gamma}\left(\partial_{t} \mathrm{e}^{-t^{2}-2 z t} t^{a}\right) \mathrm{d} t
\end{aligned}
$$

respectively.

We can also deduce the second representation from the first by the symmetry involving the multiplication by $\mathrm{e}^{z^{2}}$ and the change of variables $z \mapsto \mathrm{i} z$.

\subsection{Canonical Forms}

The natural weight of the Hermite operator equals $\mathrm{e}^{-z^{2}}$, so that

$$
\mathcal{S}_{\lambda}=\mathrm{e}^{z^{2}} \partial_{z} \mathrm{e}^{-z^{2}} \partial_{z}-2 \lambda-1 \text {. }
$$

The balanced (as well as Schrödinger-type) form of the Hermite operator is

$$
\mathrm{e}^{-\frac{z^{2}}{2}} \mathcal{S}_{\lambda} \mathrm{e}^{\frac{z^{2}}{2}}=\partial_{z}^{2}-z^{2}-2 \lambda .
$$

Note that the symmetry $(z, \lambda) \mapsto(\mathrm{i} z,-\lambda)$ is obvious in the balanced form.

Remark 7.2. The balanced form of the Hermite equation is known in the literature as the Weber or parabolic cylinder equation. It is usually written in one of two forms

$$
\partial_{z}^{2}-\frac{1}{4} z^{2}-k, \quad \partial_{z}^{2}+\frac{1}{4} z^{2}-k
$$

\subsection{Even Solution}

Inserting a power series in the equation, we see that the Hermite equation has an even solution

$$
\begin{aligned}
S_{\lambda}^{+}(z) & :=\sum_{j=0}^{\infty} \frac{\left(\frac{a}{2}\right)_{j}}{(2 j) !}(2 z)^{2 j} \\
& =F\left(\frac{a}{2} ; \frac{1}{2} ; z^{2}\right)=F_{-\frac{1}{2}, \lambda}\left(z^{2}\right) .
\end{aligned}
$$

It is the unique solution satisfying

$$
S_{\lambda}^{+}(0)=1, \quad \frac{\mathrm{d}}{\mathrm{d} z} S_{\lambda}^{+}(0)=0 .
$$

It has the properties

$$
S_{\lambda}^{+}(z)=S_{\lambda}^{+}(-z)=\mathrm{e}^{z^{2}} S_{-\lambda}^{+}(\mathrm{i} z) .
$$




\subsection{Odd Solution}

The Hermite equation has an odd solution

$$
\begin{aligned}
S_{\lambda}^{-}(z) & :=\sum_{j=0}^{\infty} \frac{\left(\frac{a+1}{2}\right)_{j}}{(2 j+1) !}(2 z)^{2 j+1}, \\
& =2 z F\left(\frac{a+1}{2} ; \frac{3}{2} ; z^{2}\right)=2 z F_{\frac{1}{2}, \lambda}\left(z^{2}\right) .
\end{aligned}
$$

It is the unique solution of the Hermite equation satisfying

$$
S_{\lambda}^{-}(0)=0, \quad \frac{\mathrm{d}}{\mathrm{d} z} S_{\lambda}^{-}(0)=2 .
$$

It has the properties

$$
S_{\lambda}^{-}(z)=-S_{\lambda}^{-}(-z)=-\mathrm{ie}^{z^{2}} S_{-\lambda}^{-}(\mathrm{i} z)
$$

\subsection{Standard Solutions}

The Hermite equation has only one singular point, $\infty$. We will see that one can define two kinds of solutions with a simple asymptotics at $\infty$.

By Theorem 7.1, for appropriate $\gamma_{1}$ and $\gamma_{2}$, the following integrals are solutions:

$$
\begin{gathered}
\int_{\gamma_{1}} \mathrm{e}^{-t^{2}-2 t z} t^{\lambda-\frac{1}{2}} \mathrm{~d} t, \\
\int_{\gamma_{2}} \mathrm{e}^{t^{2}}(z-t)^{-\lambda-\frac{1}{2}} \mathrm{~d} t .
\end{gathered}
$$

In the first case the integrand has a singular point at 0 and goes to zero as $t \rightarrow \pm \infty$. We can thus use $\gamma_{1}$ with such endpoints. We will see that they give all standard solutions.

In the second case the integrand has a singular point at $z$ and goes to zero as $t \rightarrow \pm$ i $\infty$. Using $\gamma_{2}$ with such endpoints, we will also obtain all standard solutions.

7.10.1. Solution $\sim z^{-a}$ for $z \rightarrow+\infty$. The following function is the solution of the Hermite equation that behaves as $z^{-a}=z^{-\lambda-\frac{1}{2}}$ for $|z| \rightarrow \infty,|\arg z|<$ $\pi / 2-\epsilon$ :

$$
S_{\lambda}(z):=z^{-\lambda-\frac{1}{2}} \tilde{F}_{-\frac{1}{2}, \lambda}\left(-z^{-2}\right)=z^{-a} F\left(\frac{a}{2}, \frac{a+1}{2} ;-;-z^{-2}\right) .
$$

We will also introduce alternatively normalized solutions:

$$
\begin{aligned}
\mathbf{S}_{\lambda}^{\mathrm{I}}(z) & :=2^{-\lambda-\frac{1}{2}} \Gamma\left(\lambda+\frac{1}{2}\right) S_{\lambda}(z) \\
& =2^{-a} z^{-a} \frac{1}{\Gamma(a)} F\left(\frac{a}{2}, \frac{a+1}{2} ;-;-z^{-2}\right), \\
\mathbf{S}_{\lambda}^{0}(z) & :=\sqrt{\pi} S_{\lambda}(z) .
\end{aligned}
$$


(The normalization of $\mathbf{S}_{\lambda}^{0}$ is somewhat trivial - we introduce it to preserve the analogy with the Gegenbauer equation, which had a less trivially normalized solution $\mathbf{S}_{\alpha, \lambda}^{0}$ )

Assuming that $z \notin]-\infty, 0$ ], we have an integral representation valid for $-\frac{1}{2}<\lambda$ :

$$
\int_{0}^{\infty} \mathrm{e}^{-t^{2}-2 t z} t^{\lambda-\frac{1}{2}} \mathrm{~d} t=\mathbf{S}_{\lambda}^{\mathrm{I}}(z)
$$

and for all parameters:

$$
-\mathrm{i} \int_{]-\mathrm{i} \infty, z^{-, i} \infty[} \mathrm{e}^{t^{2}}(z-t)^{-\lambda-\frac{1}{2}} \mathrm{~d} t=\mathbf{S}_{\lambda}^{0}(z) .
$$

7.10.2. Solution $\sim(-\mathrm{i} z)^{a-1} \mathrm{e}^{z^{2}}$ for $z \rightarrow+\mathrm{i} \infty$. The following function is the solution of the Hermite equation that behaves as $(-\mathrm{i} z)^{a-1} \mathrm{e}^{z^{2}}=(-\mathrm{i} z)^{\lambda-\frac{1}{2}} \mathrm{e}^{z^{2}}$ for $|z| \rightarrow \infty,|\arg z-\pi / 2|<\pi / 2-\epsilon$ :

$$
\mathrm{e}^{z^{2}} S_{-\lambda}(-\mathrm{i} z)=(-\mathrm{i} z)^{\lambda-\frac{1}{2}} \mathrm{e}^{z^{2}} \tilde{F}_{-\frac{1}{2},-\lambda}\left(z^{-2}\right) .
$$

Assuming that $z \notin[0, \infty[$, we have an integral representation valid for all parameters:

$$
\int_{-\infty, 0^{+}, \infty[} \mathrm{e}^{-t^{2}-2 t z}(-\mathrm{i} t)^{\lambda-\frac{1}{2}} \mathrm{~d} t=\mathrm{e}^{z^{2}} \mathbf{S}_{-\lambda}^{0}(-\mathrm{i} z),
$$

and for $\lambda<\frac{1}{2}$ :

$$
-\mathrm{i} \int_{[z, \mathrm{i} \infty[} \mathrm{e}^{t^{2}}(-\mathrm{i}(t-z))^{-\lambda-\frac{1}{2}} \mathrm{~d} t=\mathrm{e}^{z^{2}} \mathbf{S}_{-\lambda}^{\mathrm{I}}(-\mathrm{i} z) .
$$

\subsection{Connection Formulas}

We can decompose the standard solutions into the even and odd solutions:

$$
\begin{aligned}
S_{\lambda}(z) & =\frac{\sqrt{\pi}}{\Gamma\left(\frac{2 \lambda+3}{4}\right)} S_{\lambda}^{+}(z)-\frac{\sqrt{\pi}}{\Gamma\left(\frac{2 \lambda+1}{4}\right)} S_{\lambda}^{-}(z) ; \\
\mathrm{e}^{z^{2}} S_{-\lambda}(-\mathrm{i} z) & =\frac{\sqrt{\pi}}{\Gamma\left(\frac{3-2 \lambda}{4}\right)} S_{\lambda}^{+}(z)+\mathrm{i} \frac{\sqrt{\pi}}{\Gamma\left(\frac{1-2 \lambda}{4}\right)} S_{\lambda}^{-}(z) .
\end{aligned}
$$

\subsection{Recurrence Relations}

The following recurrence relations follow easily from the commutation properties of Sect. 7.4:

$$
\begin{aligned}
\partial_{z} S_{\lambda}(z) & =-\left(\frac{1}{2}+\lambda\right) S_{\lambda+1}(z), \\
\left(\partial_{z}-2 z\right) S_{\lambda}(z) & =-2 S_{\lambda-1}(z), \\
\left(z \partial_{z}+\frac{1}{2}-\lambda-2 z^{2}\right) S_{\lambda}(z) & =-2 S_{\lambda-2}(z),
\end{aligned}
$$




$$
\left(z \partial_{z}+\frac{1}{2}+\lambda\right) S_{\lambda}(z)=-\frac{1}{2}\left(\frac{1}{2}+\lambda\right)\left(\frac{3}{2}+\lambda\right) S_{\lambda+2}(z)
$$

\subsection{Hermite Polynomials}

If $-a=n=0,1,2, \ldots$, then Hermite functions are polynomials.

Following Sect. 1.6, they can be defined by the following version of the Rodriguez-type formula:

$$
H_{n}(z):=\frac{(-1)^{n}}{n !} \mathrm{e}^{z^{2}} \partial_{z}^{n} \mathrm{e}^{-z^{2}}
$$

Remark 7.3. The Hermite polynomials found usually in the literature equal

$$
n ! H_{n}(z) .
$$

The advantage of our convention is that the Rodriguez-type formula has the same form for all classes of hypergeometric type polynomials.

The differential equation:

$$
\left(\partial_{z}^{2}-2 z \partial_{z}+2 n\right) H_{n}(z)=\left(-n ; z, \partial_{z}\right) H_{n}(z)=0
$$

The generating function:

$$
\exp \left(2 t z-t^{2}\right)=\sum_{n=0}^{\infty} t^{n} H_{n}(z) .
$$

The integral representation:

$$
H_{n}(z)=\frac{1}{2 \pi \mathrm{i}} \int_{\left[0^{+}\right]} \exp \left(2 t z-t^{2}\right) t^{-n-1} \mathrm{~d} t .
$$

Recurrence relations:

$$
\begin{aligned}
\partial_{z} H_{n}(z) & =2 H_{n-1}(z), \\
\left(\partial_{z}-2 z\right) H_{n}(z) & =-(n+1) H_{n+1}(z), \\
\left(z \partial_{z}-n\right) H_{n}(z) & =2 H_{n-2}(z), \\
\left(z \partial_{z}+n+1-2 z^{2}\right) H_{n}(z) & =-(n+1)(n+2) H_{n+2}(z) .
\end{aligned}
$$

The differential equation, the Rodriguez-type formula, the generating function, the integral representation and the first pair of recurrence relations are special cases of the corresponding formulas of Sect. 1.6.

We have several alternative expressions for Hermite polynomials:

$$
\begin{aligned}
H_{n}(z) & =-\lim _{\nu \rightarrow n}(-1)^{n}(\nu-n) \mathbf{S}_{-n-\frac{1}{2}}^{\mathrm{I}}(z)=\frac{2^{n}}{n !} S_{-n-\frac{1}{2}}(z) \\
& =\frac{2^{n}}{n !} z^{n} F\left(-\frac{n}{2}, \frac{-n+1}{2} ;-; z^{-2}\right) \\
& =\sum_{k=0}^{\left[\frac{n}{2}\right]} \frac{(-1)^{k}(2 z)^{n-2 k}}{k !(n-2 k) !} .
\end{aligned}
$$

Behavior at $\infty$.

$$
\lim _{n \rightarrow \infty} \frac{H_{n}(z)}{z^{n}}=\frac{2^{n}}{n !}
$$


Initial conditions at 0 .

$$
\begin{aligned}
& H_{2 m}(0)=\frac{(-1)^{m}}{m !}, \quad H_{2 m}^{\prime}(0)=0, \\
& H_{2 m+1}(0)=0, \quad H_{2 m+1}^{\prime}(0)=\frac{(-1)^{m} 2}{m !} .
\end{aligned}
$$

Identities for even and odd polynomials.

$$
\begin{aligned}
H_{2 m}(z) & =\frac{(-1)^{m} 2^{2 m} m !}{(2 m) !} L_{m}^{-1 / 2}\left(z^{2}\right)=\frac{(-1)^{m}(2 z)^{2 m} m !}{(2 m) !} B_{m}^{-2 m-\frac{1}{2}}\left(-z^{-2}\right), \\
& =\frac{(-1)^{m}}{m !} S_{-2 m-\frac{1}{2}}^{+}(z)=\frac{(-1)^{m}}{m !} F\left(-m ; \frac{1}{2} ; z^{2}\right), \\
H_{2 m+1}(z) & =\frac{(-1)^{m} 2^{2 m+1} m !}{(2 m+1) !} z L_{m}^{1 / 2}\left(z^{2}\right)=\frac{(-1)^{m}(2 z)^{2 m+1} m !}{(2 m+1) !} B_{m}^{-2 m-\frac{3}{2}}\left(-z^{-2}\right) \\
& =\frac{(-1)^{m}}{m !} S_{-2 m-\frac{3}{2}}^{-}(z)=\frac{(-1)^{m}}{m !} 2 z F\left(-m ; \frac{3}{2} ; z^{2}\right) .
\end{aligned}
$$

\section{Acknowledgements}

I acknowledge the help of Laurent Bruneau, Michał Godliński, and especially Michał Wrochna and Przemysław Majewski who proofread parts of previous versions of this work. The research of the author was supported in part by the National Science Center (NCN) Grant No. 2011/01/B/ST1/04929.

Open Access. This article is distributed under the terms of the Creative Commons Attribution License which permits any use, distribution, and reproduction in any medium, provided the original author(s) and the source are credited.

\section{Appendix A. Contours for Integral Representations}

In this appendix, we collect contours used in various integral representations of hypergeometric type functions.

For each basic type of integral representations considered in our text, we give at least one contour for every standard representation. We give the priority to type (a) contours. If they are unavailable, we show a type (b) contour. In some cases, we present both a type (a) and type (b).

We also show contours that yield the degenerate solutions and the polynomial solutions. They are given by closed loops.

Here is the explanation of basic elements of our figures: 
singularity of the integrand

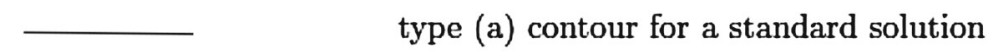

type (b) contour for a standard solution

contour for a degenerate solution

contour for a polynomial solution

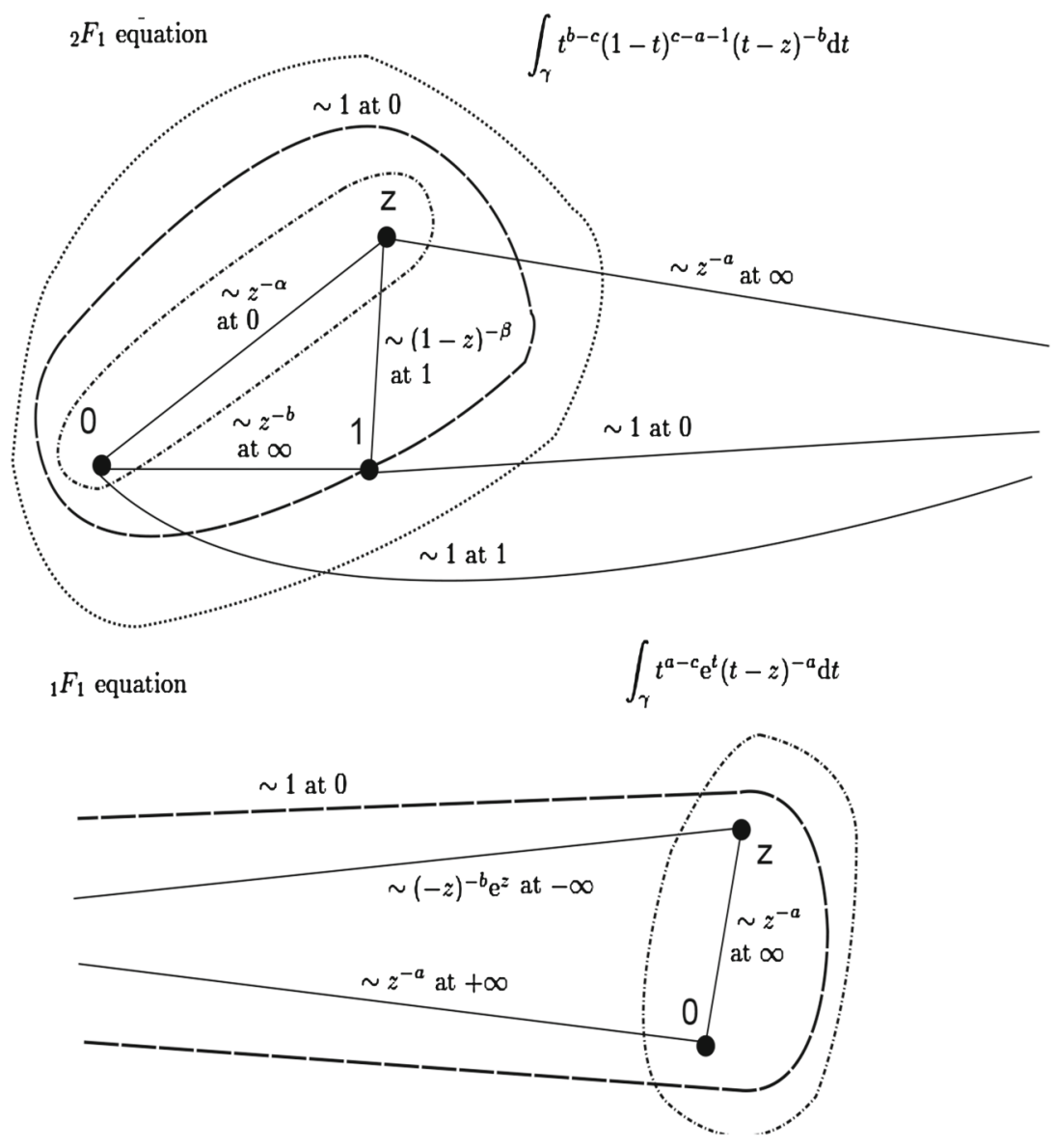


${ }_{1} F_{1}$ equation

$$
\int_{\gamma} \mathrm{e}^{\frac{i}{t}} t^{-c}(1-t)^{c-a-1} \mathrm{~d} t
$$
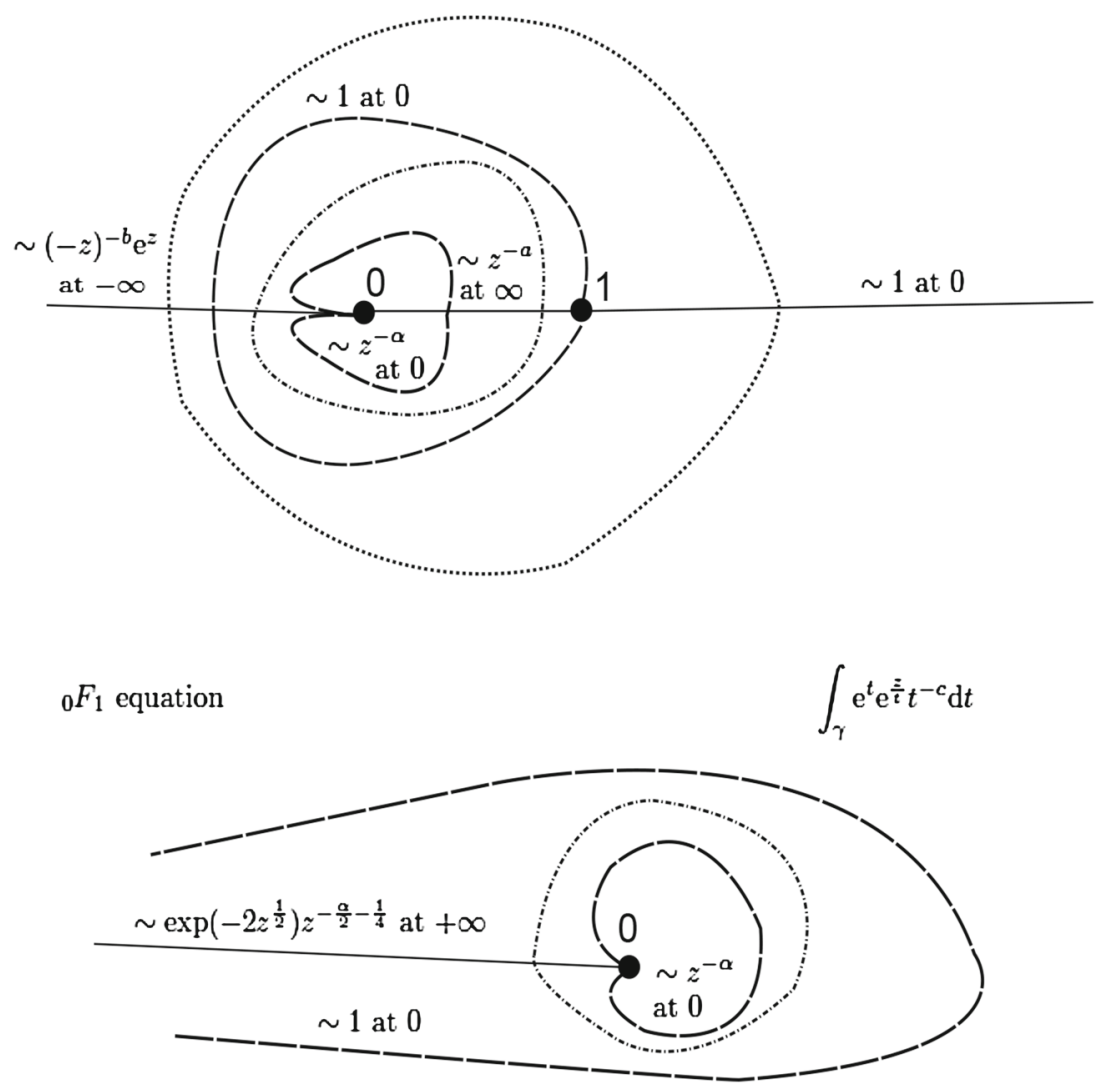


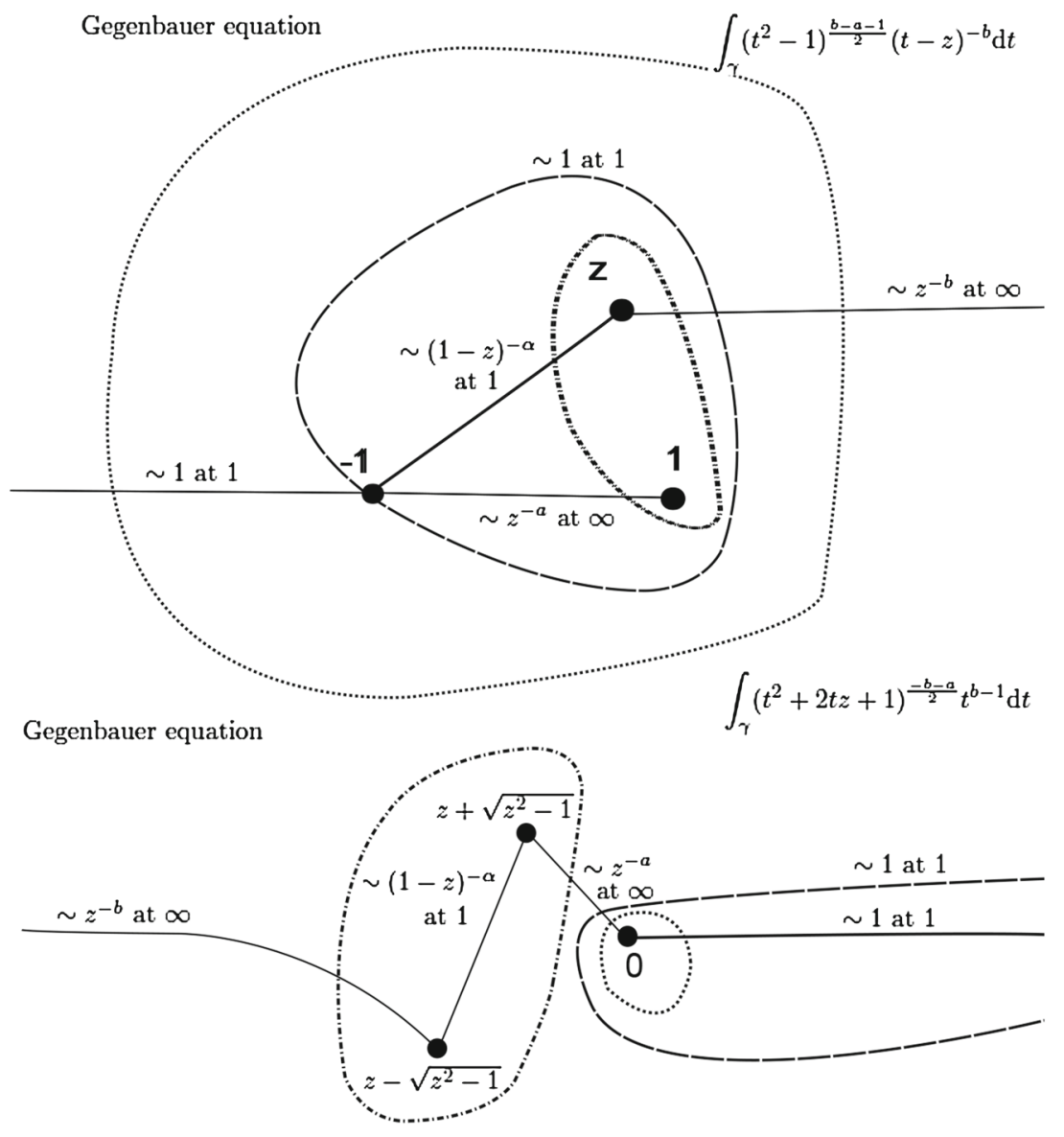


Hermite equation

$\int_{\gamma} \mathrm{e}^{-t^{2}-2 z t} t^{a-1} \mathrm{~d} t$

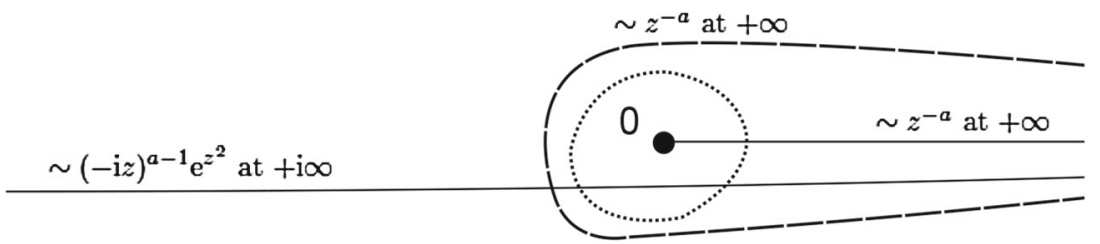

Hermite equation

$$
\int_{\gamma} \mathrm{e}^{t^{2}}(t-z)^{-a} \mathrm{~d} t
$$

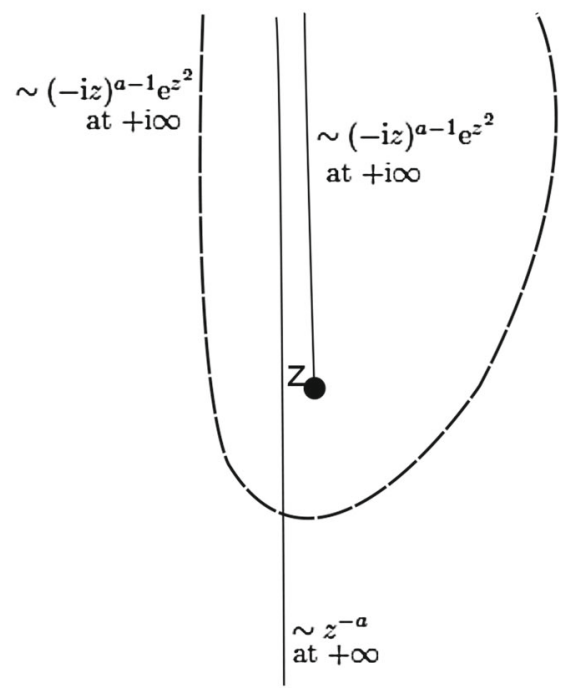

\section{References}

[1] Andrews, G.E., Askey, R., Roy, R.: Special functions. Cambridge University Press, Cambridge (1999)

[2] Dereziński, J., Majewski, P.: Conformal symmetries and hypergeometric type functions (in preparation)

[3] Erdelyi, A., Magnus, W., Oberhettinger, F., Tricomi, F.G.: Higher Transcendental Functions, vols. I, II, III. McGraw-Hill, New York (1953)

[4] Hochstadt, H.: The Functions of Mathematical Physics. Wiley, New York (1971)

[5] Infeld, L., Hull, T.: The factorization method. Revs Mod. Phys. 23, 21-68 (1951)

[6] Vilenkin, N. Ja, Klimyk, A.U.: Representations of Lie Groups and Special Functions. vol 1. Kluwer, Dordrecht

[7] Magnus, W., Oberhettinger, F., Soni, R.: Formulas and Theorems for the Special Functions of Mathematical Physics. 3rd edn. Springer, New York (1966)

[8] Miller, W.: Lie Theory and Special Functions. Academic Press, New York (1968) 
[9] Miller, W.: Symmetry and Separation of Variables. Addison-Wesley, Reading (1977)

[10] Nikiforov, U.: Special functions of mathematical physics. Birkhäuser, Basel (1988)

[11] Olver, P.J.: Applications of Lie Groups to Differential Equations. Graduate Texts in Mathematics. Springer, New York (1986)

[12] Olver, F.W.J., Lozier, D.W., Boisvert, R.F., Clark, C.W. (eds.): NIST Handbook of Mathematical Functions. Cambridge University Press, Cambridge

[13] Rainville, E.D.: Special Functions. The Macmillan Co., New York (1960)

[14] Truesdell, C.: An essay toward a unified theory of special functions based upon the functional equation $\partial F(z, \alpha) / \partial z=F(z, \alpha+1)$. Annals of Mathematical Studies no. 18. Princeton University Press, Princeton (1948)

[15] Vilenkin, N.Ya.: Special Functions and the Theory of Group Representations. Translations of Mathematical Monographs. AMS, Providence (1968)

[16] Wawrzyńczyk, A.: Modern Theory of Special Functions. PWN, Warszawa (1978) (Polish)

[17] Whittaker, E.T., Watson, G.N.: A course of Modern Analysis, vols. I, II, 4th edn. (reprint of 1927 ed.). Cambridge University Press, New York (1962)

Jan Dereziński

Department of Mathematical Methods in Physics

Faculty of Physics

University of Warsaw

Hoża 74, 00-682 Warsaw, Poland

e-mail: jan.derezinski@fuw.edu.pl

Communicated by Claude Alain Pillet.

Received: November 2, 2012.

Accepted: May 14, 2013. 